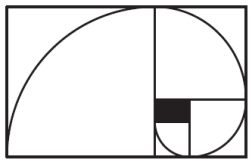

2005, Volume 10, 1-100

https://doi.org/10.21711/217504322005/em101

\title{
A January 2005 invitation to random groups
}

\section{Yann Ollivier}

\begin{abstract}
Random groups provide a rigorous way to tackle such questions as "What does a typical (finitely generated) group look like?" or "What is the behavior of an element of a group when nothing particular happens?"

We review the results obtained on random groups as of January 2005. We give proper definitions and list known properties of typical groups. We also emphasize properties of random elements in a given group. In addition we present more specific, randomly twisted group constructions providing new, "wild" examples of groups.

A comprehensive discussion of open problems and perspectives is included.
\end{abstract}

2000 Mathematics Subject Classification: 20F65, 20P05, 60B99, 20F05, $20 \mathrm{~F} 67$. 



\section{Foreword}

Our aim here is to present, within the limited scope of the author's knowledge, the state of the art of random groups. The decision to write such a survey arose from consideration of the rapidly growing number of publications on the subject, which, from a bunch of theorems, is slowly shaping into a theory. A whole section has been devoted to the statement of open problems of various difficulty.

The accompanying "primer to geometric group theory" is meant as a gentle introduction to the necessary background material, may readers outside of the field find some appeal in random groups.

There are no proofs in this book, except that of the foundational density $1 / 2$ phase transition theorem, which constitutes a standalone chapter at the end of the text. Most results are indeed very technical and gathering all proofs would have resulted in a heavy (in all senses of the word) treatise rather than an "invitation".

The goal was not to track down the origins of the generic way of thinking in group theory or neighboring fields, but to review the results in that branch of mathematics which treats of the groups obtained from random presentations. In particular, and mainly because of the author's incompetence on these matters, the asymptotic theory of finite groups and properties of random elements therein are not covered.

The information presented here has deliberately been limited to the works available to the author as of January $31^{\text {st }}, 2005$, except for bibliographical references to then unpublished manuscripts, which have been updated for the reader's convenience.

The roots of all current mathematical work related to random groups lie unquestionably in Misha Gromov's fertile mind, and can be traced back to his seminal 1987 paper [Gro87] on hyperbolic groups. In order to illustrate the importance of his newly defined [Gro78, Gro83] class of groups, he stated (without proof) that "most" groups with a fixed number of generators and relations and "long enough" relation length are hyperbolic (see § I.1.).

He later substantiated his thoughts on the subject in Chapter 9 of [Gro93], entitled Finitely presented groups: density of random groups and other speculations, where the density model of random groups is defined and the intuition behind it thoroughly discussed. This model allows a sharp control of the quantity of relations put in a random group, and has proven very fruitful over the years, especially since the properties obtained vary with density (cf. § I.2.). 
The subject received considerable attention from the general mathematical community (see e.g. [Ghy03, Pan03]) when Gromov published his Random walk in random groups [Gro03] (elaborating on the equally renowned Spaces and questions [Gro00]), in which he uses random methods to build a group with "wild" geometric properties linked to the Baum-Connes conjecture with coefficients (see $\S$ III.2.), although these partially random groups have no pretention at all to model a "typical" group.

The first motivation for the study of random groups is the following somewhat philosophical question: "What does a typical group look like?" This theme is addressed in $\S$ I., Models of typical groups, where known properties of those are discussed. The word "typical" here is used as a convenient loose term interpolating between "random", which entails a probabilistic setting, and "generic", rather implying a topological framework. The latter is specifically developed in $\S$ I.4., where some results on the space of all marked groups are presented.

A slightly different approach is to look at properties of "typical" elements in a given group, either for themselves or in order to achieve certain goals. This is the theme of $\S$ II., Typical elements in a group. For example, a lot of "unrelated" "typical" elements in a hyperbolic group can be killed without harming too much the group (§ II.1.); this intuition has been present since the very beginning of hyperbolic group theory. Also, considering that typical relations in a presentation do not exhibit any special structure led to a sharp evaluation of the number of different one-relator groups (§ II.3.).

But random groups now have found applications to other fields of mathematics. Indeed, the use of random ingredients in constructions specifically designed to achieve certain goals allows to prove existence of groups with new properties, which are counterexamples to open questions, such as Gromov's celebrated group ( $\S$ III.2.) whose Cayley graph admits no uniform embedding into the Hilbert space, or a bunch of new groups with property $(T)$ and somewhat unexpected properties (§ III.3.). Though these groups cannot pretend to be good candidates for "typicality", they are definitely of interest to people in and outside of group theory.

Yet for the author, the primary appeal of the field is still the study of properties of "typical" groups for themselves, rather than the applications just discussed. This is, of course, a matter of (philosophical?) taste.

Acknowledgements. I would like to thank Goulnara Arzhantseva, Vincent Beffara, Yves de Cornulier, Thomas Delzant, Christophe Deroulers, Étienne Ghys, Misha Gromov, Alice Guionnet, Frédéric Haglund, Ilya Kapovich, Richard Kenyon, Marc Mézard, Pierre Pansu, Pierre Senellart, Bruno Sévennec, Jean-Claude Sikorav, Alain Valette, Maria Eulália Vares and Dani Wise for their help and comments with preparing this book. 


\section{Notation and conventions}

$\mathbb{N}$ : the set of natural numbers, including 0 .

\#A: number of elements of the set $A$.

$F_{m}$ : free group of rank $m$ over the set of generators $a_{1}, \ldots, a_{m}$ and their formal inverses. Unless otherwise stated, we assume $m \geqslant 2$.

$\langle R\rangle$ : normal subgroup generated by the set of elements $R$.

$\left\langle a_{1}, \ldots, a_{m} \mid R\right\rangle$ : group presented by generators $a_{1}, \ldots, a_{m}$ with set of relators $R$, that is, the group $F_{m} /\langle R\rangle$.

$|w|$ : length of the word $w$.

$|D|$ : number of faces of the van Kampen diagram $D$.

$|\partial D|$ : boundary length of the van Kampen diagram $D$.

Reduced word: a word not containing any letter immediately followed by its inverse.

Non-elementary hyperbolic group: a hyperbolic group which is neither finite nor quasi-isometric to $\mathbb{Z}$.

Hyperbolicity: the fact of being non-elementary hyperbolic.

With overwhelming probability: with probability tending to 1 when some natural parameter (often denoted $\ell$ ) tends to infinity. 


\section{Contents}

A primer to geometric group theory 9

Free groups, group presentations . . . . . . . . . . . 10

Cayley graphs and complexes . . . . . . . . . . . . . . . 12

Groups as geometric objects . . . . . . . . . . . . . . . 14

Van Kampen diagrams . . . . . . . . . . . . . . . . . . 15

Hyperbolic groups . . . . . . . . . . . . . . . . . 17

Suggested reading . . . . . . . . . . . . . . 21

A January 2005 invitation to random groups 25

I. Models of typical groups 26

I.1. Forerunners: few-relator models . . . . . . . . . . . . 27

I.2. Gromov's density . . . . . . . . . . . . . . . . . . 29

I.2.a. Definition of density. . . . . . . . . . . . . . . . 29

I.2.b. The phase transition. . . . . . . . . . . . 31

I.2.c. Variations on the model. . . . . . . . . . . . . . . . 32

I.3. Critical densities for various properties _. . . . . . . . 33

I.3.a. Van Kampen diagrams and small cancellation properties. 33

I.3.b. Dimension of the group. . . . . . . . . . . . . . 35

I.3.c. Algebraic properties at density 0: rank, free subgroups. $\quad 35$

I.3.d. Boundary and geometric properties of the Cayley graph. $\quad 37$

I.3.e. Growth exponent. . . . . . . . . . . . . . . 38

I.3.f. Random walk in a typical group. . . . . . . . . . . . . 39

I.3.g. Property $(T)$ and the triangular model. . . . . . . . . 40

I.3.h. Testing the triangular model: Gromov vs. the computer. $\quad 42$

I.3.i. Cubical CAT(0)-ness and the Haagerup property. . . . 43

I.4. The space of marked groups . . . . . . . . . . . . . . 44

II. Typical elements in a group 48

II.1. Killing random elements of a group . . . . . . . . . . 48

II.1.a. Random quotients by elements in a ball. . . . . . . . . 48

II.1.b. Growth of random quotients. . . . . . . . . . . . . 49

II.2. Killing random words, and iterated quotients . . . . . . . 50

II.2.a. Random quotients by words. . . . . . . . . . . 50

II.2.b. Harmful torsion. . . . . . . . . . . . . . . . . . . 51

II.2.c. Cogrowth of random quotients, and iterated quotients. . 52

II.3. Counting one-relator groups . . . . . . . . . . . . . . . . 53 


\section{Contents}

III. Applications: Random ingredients in specific construc-

tions

III.1. Shaping Cayley graphs: graphical presentations . . . . . . . 56

III.1.a. Labelled graphs and group presentations. . . . . . . . 56

III.1.b. Graphical small cancellation. . . . . . . . . . . . . . 57

III.1.c. Random labellings are $G r^{\prime}(1 / 6)$. . . . . . . . . . . . 58

III.1.d. Random labellings of expanders entail Kazhdan's prop$\operatorname{erty}(T) \ldots \ldots \ldots \ldots \ldots$. . . . . . . . . 59

III.1.e. Generalizations: relative graphical presentations, and more. 60

III.2. Cayley graphs with expanders . . . . . . . . . . . . . . . 61

III.3. Kazhdan small cancellation groups? . . . . . . . . . . . . . 65

$\begin{array}{ll}\text { IV. Open problems and perspectives } & 68\end{array}$

IV.a. What happens at the critical density? . . . . . . 68

IV.b. Different groups at different densities? . . . . . . . . 69

IV.c. To $(T)$ or not to $(T) . \ldots \ldots \ldots$. . . . . . . 70

IV.d. Rank and boundary. . . . . . . . . . . . 71

IV.e. More properties of random groups. . . . . . . . . . 71

IV.f. The world of random quotients. . . . . . . . . . . . 72

IV.g. Dynamics on the space of marked groups. . . . . . . 73

IV.h. Isoperimetry and two would-be classes of groups. . . . . 73

IV.i. Metrizing Cayley graphs, generalized small cancellation and "rotation families". . . . . . . . . . . . . . . . . . 75

IV.j. Better Cayley graphs with expanders? . . . . . . . . 76

IV.k. The temperature model and local-global principles. . . . 76

IV.l. Random Lie algebras. . . . . . . . . . . . . . 79

IV.m. Random Abelian groups, computer science and statistical physics. . . . . . . . . . . 79

V. Proof of the density one half theorem 81

V.a. Prolegomena. . . . . . . . . . . 81

V.b. Probability to fulfill a diagram. . . . . . . . . . 83

V.c. The local-global principle, or Gromov-Cartan-Hadamard theorem. . . . . . . . . . . . 86

V.d. Infiniteness. . . . . . . . . . 87

$\begin{array}{ll}\text { Bibliography } & 89\end{array}$ 
A JANUARY 2005 INVITATION TO RANDOM GROUPS 
A primer to geometric group theory 


\section{A primer to geometric group theory}

This part of the book is aimed at those not so familiar with geometric group theory. It covers the necessary material for understanding what random groups are all about (though it can be read without any interest in random groups), namely: basic facts on group presentations and free groups; the construction of Cayley graphs and complexes; an introduction to the geometric viewpoint in group theory; a sketch of what van Kampen diagrams are; and the different definitions of hyperbolic groups. An explicit example then gathers it all in two pictures.

We do not pretend to give an overview of geometric group theory as a whole: many important aspects of the field are not mentioned, and focus is primarily placed on the material somehow relevant to random groups.

A few selected references are given at the end as suggested reading.

\section{Free groups, group presentations}

From a geometric group theorist's viewpoint, which may not be everyone's, the simplest of all groups are free groups over some set of generators.

Let $S_{+}$be any set (frequently $S_{+}=\left\{a_{1}, \ldots, a_{m}\right\}$ is finite). Intuitively speaking, the free group on $S_{+}$is the group consisting of all formal products of elements of $S_{+}$and their formal inverses, with the cancellation $x x^{-1}=$ $x^{-1} x=e$ as the only computation rule.

More precisely, let $S_{-}$be the set of formal inverses $x^{-1}$ of the elements $x \in S$, which is just a distinct copy of $S_{+}$, and let $S=S_{+} \sqcup S_{-}$. For $n \in \mathbb{N}$, a word of length $n$ on the alphabet $S$ is a sequence of $n$ elements of $S$. In the particular case $n=0$ there is only one such sequence, called the empty word. A word on $S$ is just a word of any length, i.e. an element of $\bigsqcup_{n=0,1,2 \ldots} S^{n}$.

A word is said to be reduced if it does not contain as a subword any sequence of the form $x x^{-1}$ or $x^{-1} x$, for any $x \in S$. With any word can be associated a reduced word, by iterated removal of all such cancellable pairs (the reduced word obtained does not depend on the order in which removals are performed), an operation called reduction.

The free group generated by $S$ (or by $S_{+}$, terminology is floppy) has all reduced words on $S$ as its elements. Multiplication is simply the concate- 
nation of words, followed by reduction if necessary. The neutral element $e$ is the empty word.

Of special importance is the case when $S=\left\{a_{1}, \ldots, a_{m}\right\}$ is finite; the corresponding free group is denoted by $F_{m}$. When $m=1$ it is isomorphic to the group of integers $\mathbb{Z}$.

Now any group can be seen as a free group but with more "computation rules" than simply $x x^{-1}=x^{-1} x=e$. This gives rise to the notion of group presentations: a group specified by a given set of generators $S$, with some "enforced" computation rules. For example, the presentation $\left\langle a \mid a^{3}=e\right\rangle$ (read: the group generated by $a$, knowing that $a^{3}=e$ ) defines a group isomorphic to $\mathbb{Z} / 3 \mathbb{Z}$.

Namely, a computation rule is any equality $w_{1}=w_{2}$ where $w_{1}, w_{2}$ are words on a given alphabet $S$. Any such rule can be rewritten $w_{1} w_{2}^{-1}=e$, and so most of the time rules are specified by giving only one word $r$, with the rule $r=e$ in mind.

So let $R$ be a set of words on a given alphabet $S=S_{+} \sqcup S_{-}$. The group presented by $\left\langle S_{+} \mid\{r=e\}_{r \in R}\right\rangle$, or, more simply, by $\left\langle S_{+} \mid R\right\rangle$, is defined as follows. Start from the free group $F_{S}$ generated by $S_{+}$. The way to enforce a relation $r=e$ is to quotient $F_{S}$ by the normal subgroup generated by $r$. So let $\langle R\rangle$ be the normal closure of the subgroup of $F_{S}$ generated by all words $r \in R$. The group presented by $\left\langle S_{+} \mid R\right\rangle$ is the group $F_{S} /\langle R\rangle$. It is the "largest" group in which all relations $r=e, r \in R$, hold.

Let us give a few examples: the free group $F_{1}$ of rank one (a.k.a. $\mathbb{Z}$ ) has the presentation $\langle a \mid \varnothing\rangle$. The cyclic groups $\mathbb{Z} / n \mathbb{Z}$ are given by $\left\langle a \mid a^{n}=e\right\rangle$. The free group of rank two is $F_{2}=\langle a, b \mid \varnothing\rangle$; if we force $a$ and $b$ to commute we get $\mathbb{Z} \times \mathbb{Z}=\langle a, b \mid a b=b a\rangle$.

The elements of $S_{+}$in a presentation $\left\langle S_{+} \mid R\right\rangle$ are called generators. Those of $R$ are called relators. It is easily seen that relators can always be assumed to be reduced words.

Note that any group has some presentation, in a kind of tautological way. Let $G$ be a group and take $S_{+}=G$ i.e. all elements of $G$ will be generators. Now let the set of words $R$ consist of all products of elements of $S$ which happen to be equal to $e$ in $G$. It is easy to check that $G=\left\langle S_{+} \mid R\right\rangle$. (Of course this presentation is, in general, way too large.)

This means that free groups have a "universal" property, namely, for each group $G$ there is a set $S$ and a surjective homomorphism from a free group $F_{S}$ to $G$. More precisely, if $X \subset G$ is any set which generates $G$ together with $X^{-1}$, then there is a surjective homomorphism $F_{X} \rightarrow G$ "sending $X$ to $X$ " i.e. sending an abstract word in the generators to its image in $G$. If $\mathcal{R}$ is the kernel of this homomorphism, then any part $R \subset \mathcal{R}$ the normal closure of which is $\mathcal{R}$, will give rise to a presentation $G=\langle X \mid R\rangle$.

A group is finitely presented if it admits a presentation $\left\langle S_{+} \mid R\right\rangle$ with both $S_{+}$and $R$ finite. A group is finitely generated (or of finite type) if $S_{+}$ 
is finite.

Presentations of a given group are by no means unique. For example, the trivial group $\{e\}$ has arbitrarily (not so) stupid presentations such as $\left\langle a, b \mid a=e, b^{2} a b^{-1}=e\right\rangle$. (In fact it is not even algorithmically decidable whether a given presentation defines the trivial group or not!)

\section{Cayley graphs and complexes}

Hereafter, in order to get locally compact objects, we suppose that all sets of generators $S$ are finite.

Cayley graphs. The above may seem quite combinatorial but actually carries a geometrical meaning, which is sometimes a more natural way to think of group presentations. For example, the group given by $\left\langle a \mid a^{3}=e\right\rangle$ can be thought of as a three-edge cycle.

Let $G$ be a group generated by a set $S=S_{+} \sqcup S_{-}$. The Cayley graph of $G$ with respect to $S$ is the graph with elements of $G$ as vertices and in which edges correspond to multiplication on the right by the generators.

More precisely, the Cayley graph is an unoriented graph with some decoration. Vertices are the elements of $G$. Now for each $x \in G$ and $s \in S_{+}$, add an unoriented edge between the vertices $x$ and $x s$, so that edges are in bijection with $G \times S_{+}$(this may result in multiple edges and loops). Now keep track of this on the edges, by deciding that with each edge together with an orientation choice, will be associated a label in $S_{+} \sqcup S_{-}$; namely, the edge from $x$ to $x s$, oriented this way, will have label $s$, and label $s^{-1}$ when oriented the other way around ${ }^{1}$.

Basic examples are (w.r.t. the obvious generating sets): The Cayley graph of the free group $F_{m}$ is an infinite tree in which each vertex has valency $2 m$. The Cayley graph of the group $\mathbb{Z} \times \mathbb{Z}$ is an infinite square grid in the plane. The Cayley graph of the cyclic group of order $n$ is an $n$-edge cycle.

The Cayley graph is homogeneous: all vertices play the same role and could have been chosen to represent the neutral element $e$.

Given any vertex $x$ in the Cayley graph and any word $w$ on the alphabet $S$, we can start at $x$ and track $w$ in the graph by "following the edge labels", which brings us at $x w$. This path is called the lift of $w$ (starting at $x$ ). Note that $w$ is reduced if and only if this path has no local backtracks.

Left and right actions. The group $G$ acts on the vertices of the Cayley graph in two ways: by left or right multiplication.

Left multiplication by $g \in G$ is a graph action: it brings adjacent vertices to adjacent vertices, preserving edges. In particular it preserves the graph

\footnotetext{
${ }^{1}$ The details of these definitions guarantee that each vertex has valency $2 \times \# S_{+}$and that the Cayley graph of the cyclic group of order $n$ is an $n$-cycle even for $n=1,2$.
} 
distance. Note however that it does not bring a point to a nearby point: $x$ and $g x$ can be very far away in the graph.

Conversely, right multiplication by $g \in G$ is not, in general, a graph action, because a priori one does not pass from $x g$ to $x s g$ by multiplication by $s$. So two points linked by an edge are not mapped to two points linked by an edge: right multiplication acts only at the level of vertices of the Cayley graph. However, right multiplication by $g$ moves a given point by a distance at most the length of $g$ (since it corresponds to following the path labelled by $g$ in the Cayley graph, starting at the given point).

Consequently, when one mentions the action of $G$ on its Cayley graph, it is the left action which is meant. (Test all this in $F_{2} \ldots$ )

Cayley complexes. It is worthy to note that any word equal to $e$ in $G$ will lift to a loop in the Cayley graph, and conversely. If $G$ is given by a presentation $\left\langle S_{+} \mid R\right\rangle$, this will be the case, in particular, for the relators $r \in R$.

The Cayley complex of a presentation $G=\left\langle S_{+} \mid R\right\rangle$ is a 2-dimensional complex obtained by gluing a disk on all paths of the Cayley graph labelled by a relator $r \in R$. More precisely, to each $x \in G$ and $r \in R$, consider the lift of $r$ starting at $x$ in the Cayley graph, which is a closed path, and glue a disk along this path. Consequently the set of faces of the Cayley complex is in bijection with $G \times R$.

Basic examples are (w.r.t. the usual presentations): The Cayley complex of a free group is just its Cayley graph. The Cayley complex of $\mathbb{Z} \times \mathbb{Z}$ is the square tiling of the plane. The Cayley complex of the cyclic group of order $n$ consists of $n$ disks sharing a common boundary (a copy of the relation $a^{n}=e$ is glued to each element) $)^{2}$.

Most importantly, the Cayley complex is simply connected. Indeed, loops in the Cayley graph label words equal to the identity. Since by definition, relators generate all relations in the group, such words are exactly products of (conjugates of) relators in the presentation. We precisely added disks along loops of the Cayley graph representing the relators, making these loops homotopically trivial.

A word about classifying spaces. Here is another method to define the Cayley graph and complex. Namely, consider a group presentation $G=\left\langle S_{+} \mid R\right\rangle$. There is a standard way to get a topological space with fundamental group $G$. Start with a bouquet $B$ of $\# S_{+}$circles, that is, a graph made of one single vertex (denoted $e$ ) and $\# S_{+}$unoriented loops.

\footnotetext{
${ }^{2}$ Several authors, including [LS77], take another convention when some relator is a proper power, in order to avoid this multiplicity. This sometimes leads to contradictions in their exposition, such as in Prop. III.4.3 of [LS77]. With the convention used here, the Cayley complex is the universal covering of the 2-skeleton of the standard classifying spaces associated with the presentation.
} 
Choose an orientation for each loop and label loops bijectively by the generators in $S_{+}$; consider that the loop with reverse orientation bears the inverse label in $S_{-}$.

The fundamental group of this bouquet of circles is the free group with $\# S_{+}$generators. The universal cover of this labelled graph is exactly the Cayley graph of the free group on $S_{+}$.

Now add "relations" in the following way. Any word on the alphabet $S_{+} \sqcup S_{-}$lifts to a closed path in the labelled graph $B$, just as above for Cayley graphs. For each relator $r \in R$, add a disk to $B$ along the path labelled by $r$. In the fundamental group of $B$, this has the effect of killing the element $r$. Thus at the end, one gets a 2-complex $B$ (with one vertex, $\# S_{+}$edges and $\# R$ faces) the fundamental group of which is precisely the group $G=\left\langle S_{+} \mid R\right\rangle$. The universal cover of $B$ is exactly the Cayley complex of this presentation.

(By the way, if one goes on with this process and kill all the higherdimensional homotopy groups of $B$ by adding sufficiently many balls of dimension $\geqslant 3$, one gets a so-called classifying space for the group $G$, i.e. a space $B G$ with fundamental group $G$ and such that the universal cover $E G$ is contractible.)

\section{Groups as geometric objects}

Here again all groups are assumed to be finitely generated.

Metrics on groups. The Cayley graph of a group w.r.t. some generating set is naturally a metric space, defining each edge to have length 1 .

The combinatorial way to look at this is as follows: Given a group $G$ generated by a set of elements $S_{+}$, it is natural to define the norm $\|g\|$ of an element $g \in G$ as the smallest length of a word expressing $g$ as a product of generators in $S_{+} \sqcup S_{-}$. This coincides, of course, with the graph distance from $g$ to $e$ in the Cayley graph of $G$ w.r.t. this generating set. Note that $\|g\|=\left\|g^{-1}\right\|$, and of course $\left\|g g^{\prime}\right\| \leqslant\|g\|+\left\|g^{\prime}\right\|$.

So we get a distance function on $G$ by setting $\operatorname{dist}(g, h)$ to be the graph distance from $g$ to $h$ in the Cayley graph. Since edges of the Cayley graph correspond to right multiplication by a generator, this is the smallest length of a word $w$ such that $h=g w$, that is, $\operatorname{dist}(g, h)=\left\|g^{-1} h\right\|$. The two properties of $\|\cdot\|$ mentioned above are just the usual distance axioms.

As mentioned above, the left action of $G$ on itself preserves this metric.

Changing generators. Of course this distance depends on the chosen generating set. A finitely generated group is thus not equipped with a canonical metric, but with a family of metrics associated with all possible finite generating sets. These metrics are related in some way, which we explore now. 
Let $S_{+}=\left\{s_{1}, \ldots, s_{n}\right\}$ and $S_{+}^{\prime}=\left\{s_{1}^{\prime}, \ldots, s_{p}^{\prime}\right\}$ be two finite generating sets for a group $G$; let $\|\cdot\|$ and $\|\cdot\|^{\prime}$ be the two associated norms. We want to control $\|\cdot\|^{\prime}$ in terms of $\|\cdot\|$.

Since $S_{+}$is a generating set, each of the elements in $S_{+}^{\prime}$ has a writing in terms of the generators in $S_{+} \sqcup S_{-}$. Let $K$ be the largest length of such a writing i.e. $K=\max _{s^{\prime} \in S_{+}^{\prime}}\left\|s^{\prime}\right\|$. Since $S_{+}^{\prime}$ is finite, we have $K<\infty$.

Now suppose that some element $g$ has a writing of length $n$ in terms of $S_{+}^{\prime} \sqcup S_{-}^{\prime}$. By replacing each element of $S_{+}^{\prime}$ by a writing of it in terms of $S_{+} \sqcup S_{-}$, we get a writing of $g$ of length at most $K n$ in terms of $S_{+} \sqcup S_{-}$. So $\|\cdot\| \leqslant K\|\cdot\|^{\prime}$. The reasoning is two-sided, and so we get that the metrics defined by $S_{+}$and $S_{+}^{\prime}$ are bi-Lipschitz equivalent.

Quasi-isometries. Actually a slightly looser definition of equivalence between metric spaces than bi-Lipschitz equivalence has proven very fruitful: that of quasi-isometry. It allows, for example, the spaces $\mathbb{R}$ and $\mathbb{Z}$ to be quasi-isometric, by neglecting what happens at small scales.

Let $\left(X, d_{X}\right)$ and $\left(Y, d_{Y}\right)$ be two metric spaces. They are quasi-isometric if there exist two maps $f: X \rightarrow Y$ and $g: Y \rightarrow X$ which distort distances in a linearly controlled way and which are almost inverse to each other (up to a finite distance error). That is, there exist constants $\lambda>0, C \geqslant 0$ such that

$$
\begin{aligned}
& d_{Y}\left(f(x), f\left(x^{\prime}\right)\right) \leqslant \lambda d_{X}\left(x, x^{\prime}\right)+C \\
& d_{X}\left(g(y), g\left(y^{\prime}\right)\right) \leqslant \lambda d_{Y}\left(y, y^{\prime}\right)+C \\
& d_{X}(g(f(x)), x) \leqslant C \\
& d_{Y}(f(g(y)), y) \leqslant C
\end{aligned}
$$

for all $x, x^{\prime} \in X, y, y^{\prime} \in Y$. This is an equivalence relation.

This notion is relevant for unbounded spaces only: any bounded metric space is quasi-isometric to a point.

A change in the generating set of a group is in particular a quasi-isometry. So any quasi-isometry invariant of a metric space, when applied to Cayley graphs, will provide a well-defined invariant of finitely generated groups.

\section{Van Kampen diagrams}

Van Kampen diagrams are a visual way to represent how all equalities holding in a group are derived from combinations of relators.

Let $G=\left\langle S_{+} \mid R\right\rangle$ be a group presentation. Since we only have access to elements of $G$ as products of generators, we want to know when two words represent the same element of $G$, i.e. we are interested in the set of equalities of words $x=y$ that hold in $G$. Since this can be rewritten as $x y^{-1}=e$, it is enough to determine the set of words representing the identity element of $G$ (so-called word problem). 
This problem is actually algorithmically unsolvable: there is no algorithm that, given any finite presentation and any word, always answers whether the given word is equal to $e$ in the presentation. Moreover, this already holds for some fixed groups: there exist some group presentations for which there is no algorithm which, given any word, answers whether or not it is equal to $e$ in the presentation.

A word is equal to $e$ in the group $G$ presented by $\left\langle S_{+} \mid R\right\rangle=F_{S_{+}} /\langle R\rangle$, by definition, if it lies in the kernel of the map $F_{S_{+}} \rightarrow G$, that is, in the normal subgroup $\langle R\rangle$ generated by the relators. Hence, a word $w$ is equal to $e$ in $G$ if and only if, as a word, it can be written as a product of conjugates of relators:

$$
w=\prod_{i=1}^{N} u_{i} r_{i}^{ \pm 1} u_{i}^{-1}
$$

where $r_{i} \in R$ and $u_{i}$ is any word. (The number $N$ of relators in this decomposition, which depends on $w$, will play an important role below in the definition of hyperbolic groups.) So algebraically speaking, the set of consequences of the relators is the normal closure of the subgroup they generate. Van Kampen diagrams are a visual interpretation of these products of conjugates.

Giving a topologically clean definition of van Kampen diagrams is beyond the scope of this review. Basically, the idea is to consider each relator $r \in R$ as a polygon with as many edges as letters in $r$, the edges of which are labelled with the successive letters of $r$ (the inverse of $r$ may also be used to build a polygon with reverse orientation). Here is the polygon associated with the simple relator $a b a^{-1} b^{-1}$ (starting at bottom left corner, counterclockwise):

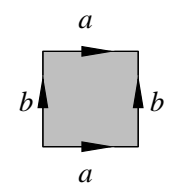

Now polygons bearing (same or different) relators can be glued to each other along edges bearing the same letter. Van Kampen diagrams are the figures resulting from such connected, simply connected gluings of relatorbearing polygons. For example, the following van Kampen diagram w.r.t. the presentation $\langle a, b \mid a b=b a\rangle$ (i.e. with the only relator $a b a^{-1} b^{-1}$ ) is a visual proof that if $a$ commutes with $b$, then so does $a^{2}$. 


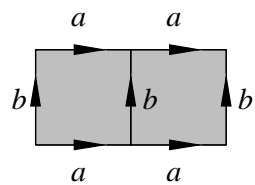

An important theorem of van Kampen states that the boundary words of such planar diagrams are exactly those words equal to $e$ in the presentation.

The connection with products of conjugates of relators is clear on the following picture, which builds the boundary word $b^{-1} a^{2} b a^{-2}$ of the van Kampen diagram given above (starting at top left corner, counterclockwise) as a product $b^{-1} r b\left(a b^{-1}\right) r\left(a b^{-1}\right)^{-1}$ of conjugates of the relator $r=a b a^{-1} b^{-1}$. Shrinking this diagram (by identifying edges arising from the same point with the same label) produces the two-square one above.

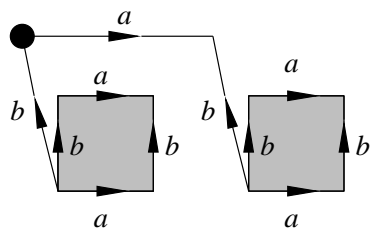

Incidentally, coming back to the algorithmic decidability problems mentioned above, we see that the word problem is semi-decidable: if a word is indeed equal to $e$, then, searching through all van Kampen diagrams, we will eventually find one decomposing the word as a product of conjugates of relators; but proving that the word is not equal to $e$ would a priori require examination and rejection of all possible diagrams.

\section{Hyperbolic groups}

A class of groups the interest for which has never declined over the years since its introduction by Gromov is that of hyperbolic groups. They have a combinatorial definition, word hyperbolicity, and a geometric one, $\delta$ hyperbolicity. Since (spoiler!) these two notions have turned out to be equivalent, we simply use the term hyperbolic.

Word hyperbolicity. Let $G=\left\langle a_{1}, \ldots, a_{m} \mid R\right\rangle$ be a group with finite presentation. Recall that any word $w$ equal to $e$ in $G$ can be written as a product

$$
w=\prod_{i=1}^{N} u_{i} r_{i}^{ \pm 1} u_{i}^{-1}
$$


(generally not in a unique way). Let $N(w)$ be the minimal number of relators in such a writing of $w$. A very natural question is: How does $N(w)$ behave when the length of $w$ grows larger and larger?

The presentation is said to be word-hyperbolic if $N(w)$ grows at most linearly with the length of $w$, that is, if there exists a constant $C$ such that for any $w$ we have $N(w) \leqslant C|w|$.

It is not difficult to see that for finite presentations, this linearity does not depend on the presentation chosen for a given group $G$ : indeed, each generator in the second presentation is a product of (finitely many) generators in the first one, and each relator in the second presentation is a consequence of (can be written as products of conjugates of) finitely many relators in the first one, so that, since the number of generators and relators is finite, the constant $C$ is perturbed by at most these quantities. So this yields a well-defined notion of a word-hyperbolic group.

When thought of in terms of van Kampen diagrams, this reads as follows: If $w$ is a word equal to $e$ in the group, then there is a van Kampen diagram $D$ with $w$ as its boundary word. Now by definition $N(w)$ is the number of faces $|D|$ of this van Kampen diagram and the length of $w$ is the boundary length $|\partial D|$. So the word hyperbolicity condition rewrites as

$$
|D| \leqslant C|\partial D|
$$

which is a linear isoperimetric inequality for van Kampen diagrams. Linear isoperimetric inequalities are a negative curvature phenomenon: for example, they are satisfied by domains in the hyperbolic plane (with area standing for $|D|$ and boundary length standing for $|\partial D|$ ), but not by domains in the Euclidean plane (where boundary length grows only like the square root of area).

$\delta$-hyperbolicity. This is a more general notion defined in any geodesic space (that is, a metric space in which the distance between two points is realized by one or several paths between them, called geodesic segments). Since any graph is a geodesic space (by definition, edge-paths realize the distance), it can be applied to Cayley graphs of groups.

$\delta$-hyperbolicity is a way to measure how "negatively curved" or "tree-like" a space looks at large scale. In a traditional negative curvature setting, triangles have the property that the sum of their angles is less than $2 \pi$ : they are curved inwards. This is measured by Rips' condition of thinness of triangles.

A triangle in a geodesic space is specified by a triple of points $(x, y, z)$ together with three geodesic segments joining them pairwise, noted $[x y]$, $[y z]$ and $[z x]$. For $\delta \geqslant 0$, the triangle is said to be $\delta$-thin if any point on $[x y]$ lies at distance at most $\delta$ from the union $[y z] \cup[z x]$ of the two other sides, and similarly for points on $[y z]$, on $[z x]$. That is, the "gap" at the middle of the triangle has size roughly $\delta$ : each side does not depart too much from the other two. 


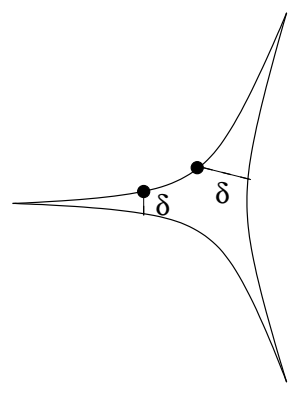

For $\delta \geqslant 0$, a geodesic space is said to be $\delta$-hyperbolic (or simply hyperbolic if $\delta$ is not specified) if any triangle in it is $\delta$-thin. Note that this must hold for all choices of geodesics between the vertices in case there are several.

The usual hyperbolic plane is hyperbolic indeed ( $\delta=10$ works). Any bounded metric space is $\delta$-hyperbolic (take its diameter as $\delta$ ). The Euclidean plane or the grid $\mathbb{Z} \times \mathbb{Z}$ are not hyperbolic for any $\delta \geqslant 0$.

Another important example is a (finite or infinite) tree, which is 0 hyperbolic (triangles are flattened). In fact, $\delta$-hyperbolic spaces are those which, "seen from far away", look like trees; they can actually by approximated by trees (up to $\delta$ ) in a very precise sense.

Now a finite group presentation is $\delta$-hyperbolic if the associated Cayley graph is. The most basic examples (apart from finite groups) are the free groups $F_{m}$, whose Cayley graphs w.r.t. the standard generating set are trees, hence 0-hyperbolic. Hyperbolic groups are thus a natural geometric generalization of free groups.

Importantly (but not obviously), $\delta$-hyperbolicity for some $\delta \geqslant 0$ is preserved under quasi-isometries, although the value of $\delta$ may change. In particular, for a group it does not depend on the choice of a generating set.

Hyperbolicity. A finitely presented group is word-hyperbolic if and only if it is $\delta$-hyperbolic for some $\delta \geqslant 0$. This is a non-trivial theorem and may actually be the shortest way to show that $\delta$-hyperbolicity does not depend on the presentation of a given group (though the value of $\delta$ does).

Note that we have defined word hyperbolicity only for group presentations, whereas $\delta$-hyperbolicity makes sense in a more general context; but the notion of linear isoperimetric inequality of van Kampen diagrams can be extended to any geodesic space and is still equivalent to $\delta$-hyperbolicity.

Small cancellation. The small cancellation conditions are simple combinatorial criteria on a group presentation which imply hyperbolicity. Maybe the one most frequently encountered is the $C^{\prime}(\alpha)$ condition. Remember the idea of van Kampen diagrams: relators in a presentation are represented as 
polygons with boundary labelled by the relator. Now if two such polygons have a common subword on their boundary (more precisely, if there is a word $w$ such that $w$ is a subword of the boundary word of the first polygon and $w^{-1}$ is a subword of the boundary word of the second one), we can glue them along this subword to form a two-face van Kampen diagram.

The $C^{\prime}(\alpha)$ condition for a presentation (for $0<\alpha<1$ ) demands that any such gluing between two polygons bearing relators $r_{1}$ and $r_{2}$ in the presentation occurs along a path $w$ of length less than $\alpha$ times the infimum of the lengths of $r_{1}$ and $r_{2}$ (except for the trivial "degenerate" gluing between a polygon and the same one with inverse orientation i.e. $r_{2}=r_{1}^{-1}$ ).

If a group presentation satisfies the $C^{\prime}(1 / 6)$ condition, then the group is hyperbolic. This results from a simple Euler characteristic argument on van Kampen diagrams, which allows to show that those have enough boundary edges to ensure a linear isoperimetric inequality.

The limit case on which the significance of $1 / 6$ is clear is the standard hexagonal tiling of the plane, which satisfies $C^{\prime}(1 / 6+\varepsilon)$ for any $\varepsilon>0$ but not $C^{\prime}(1 / 6)$, and which is not hyperbolic (compare the heptagonal tiling of the hyperbolic plane).

All in one: an example. Let $G$ be the group presented by

$$
\left\langle a, b, c, d \mid a b a^{-1} b^{-1} c d c^{-1} d^{-1}=e\right\rangle
$$

which means that the only polygon appearing in van Kampen diagrams is the following octogon.

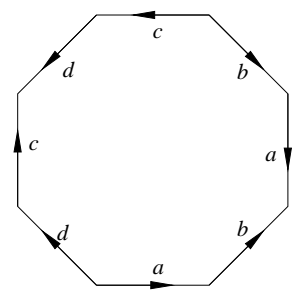

Now the only ways to glue such a polygon with a copy of itself consist in gluing the two $a$ 's, or gluing the two b's, or the two $c$ 's, or the two d's, but there is no way to find a gluing along two consecutive letters. Since the length of the relator is 8 , this means that this presentation satisfies the $C^{\prime}(1 / 8+\varepsilon)$ small cancellation condition (but not $C^{\prime}(1 / 8)$ ). Since $1 / 8<1 / 6$ the criterion above applies and so this group is hyperbolic.

This example is not anecdotic. Copies of the above octogon can be glued (in the allowed ways: an $a$ with an $a$, etc.) to form the standard octogonal tiling of the hyperbolic plane. Actually this tiling is exactly the Cayley graph of the group, which we represent on pages 22 and 23 in 
two different Euclidean views: a Poincaré model centered either on a face, emphasizing the tiling aspect, or on an element, emphasizing the Cayley graph. (When looking at the figures, keep in mind that the hyperbolic metric goes to infinity close to the disk boundary, so that all octogons are actually isometric and play the same role. The second picture is obtained from the first one by performing a hyperbolic isometry sending the left vertex of the large bottom $a$ to the center of the disk.)

Since the hyperbolic plane is hyperbolic, this is another way to check hyperbolicity of this group: the group identifies with vertices of the tiling, equipped with the edge metric, which is a discrete subset quasi-isometric to the whole hyperbolic plane. Note how tree-like the Cayley graph looks in spite of the presence of cycles of length 8 .

A group naturally acts on its Cayley graph by left multiplication and so we can take the quotient of this hyperbolic tiling by action of the group. This provides a two-dimensional object, the fundamental group of which is the group.

Let us take a closer look at this quotient. A single octogon is a fundamental domain for the action of the group on the tiling (i.e. the set of all translates of some octogon by the group exactly produces the tiling) and so the quotient is obtained by taking a single octogon and identifying its edges according to the labels. So take a single copy of the octogon above and twist it in three-dimensional space so that the two edges labelled by $a$ are identified (preserving the orientation implied by the arrows); then identify the two edges labelled by $b$, then the two ones labelled by $c$, then the ones labelled by $d$ (all eight vertices will be identified along the way). This actually leaves us with a surface of genus two, i.e. the gluing of two tori (this is quite hard to figure out - check it first in the simpler case of a square $a b a^{-1} b^{-1}$ instead of the octogon above: this square transforms into a torus).

So the group can be represented as the fundamental group of a surface of genus 2. This surface inherits a metric of negative curvature from that of the hyperbolic plane. Actually, fundamental groups of negatively curved compact surfaces, or of polyhedra with negative curvature in some combinatorial sense, were an important motivation for the introduction of hyperbolic groups and initially the main source of examples.

\section{Suggested reading}

Groups as geometric objects, hyperbolic groups, quasi-isometries, Cayley graphs...

É. Ghys, P. de la Harpe, Sur les groupes hyperboliques d'après Mikhael Gromov, Progress in Math. 83, Birkhäuser (1990).

Groups as geometric objects, quasi-isometries...

M. Gromov, Infinite groups as geometric objects, in Proceedings of the Inter- 


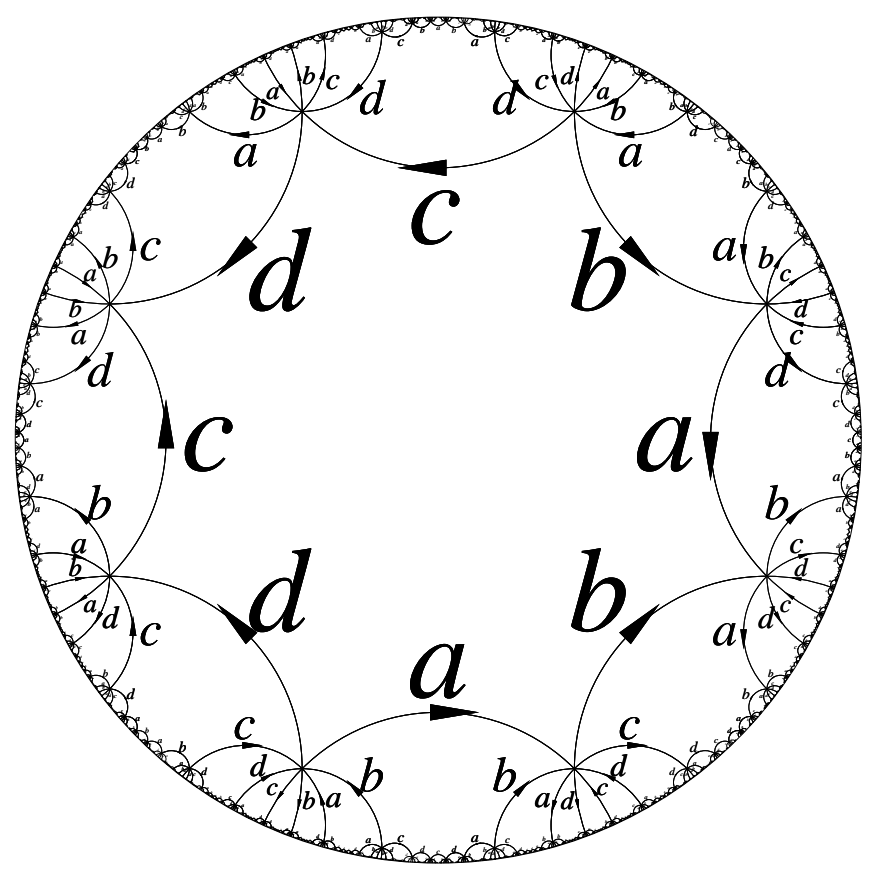




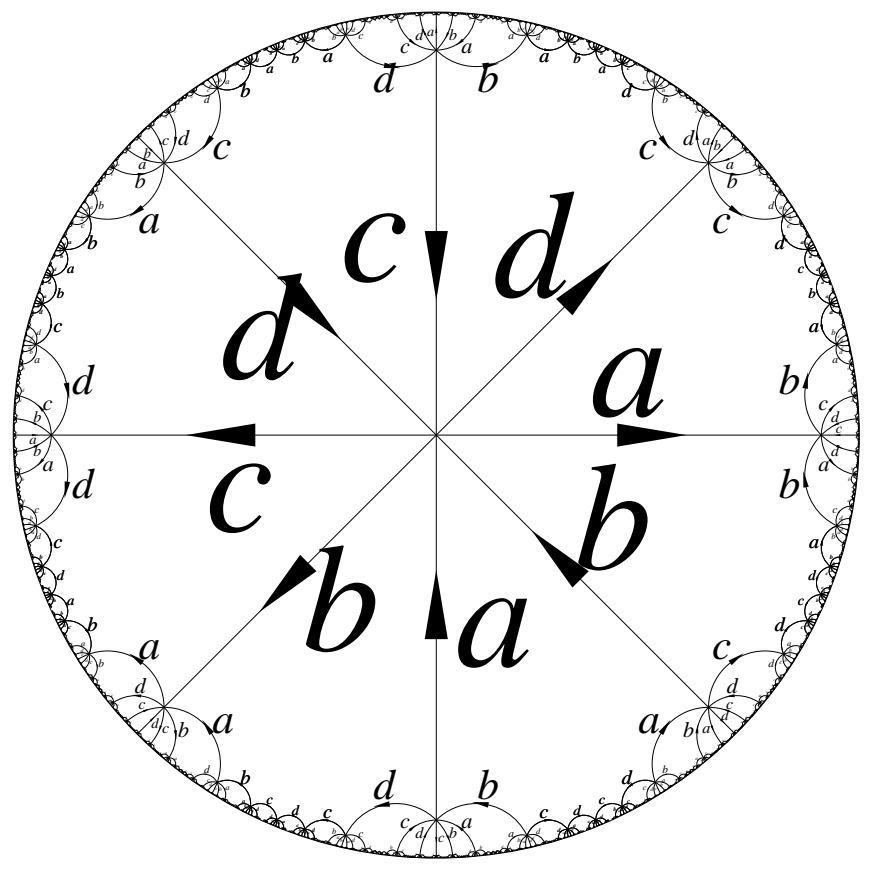


national Congress of Mathematicians, Vol. 1, 2, Warsaw (1983), 385-392, PWN, Warsaw, 1984.

Group presentations, free groups, van Kampen diagrams (under the name "cancellation diagrams"), decision problems...

J. J. Rotman, An introduction to the theory of groups, fourth edition, Graduate Texts in Mathematics 148, Springer (1995), especially chapters 11 and 12 .

Group presentations, free groups, van Kampen diagrams, small cancellation theory...

R. C. Lyndon, P. E. Schupp, Combinatorial group theory, Ergebnisse der Mathematik und ihrer Grenzgebiete 89, Springer (1977).

Negative curvature, hyperbolic groups, isoperimetric inequalities...

M. R. Bridson, A. Haefliger, Metric spaces of non-positive curvature, Grundlehren der mathematischen Wissenschaften 319, Springer (1999), especially chapters $\mathrm{H}$ and $\Gamma$. 


\section{A January 2005 invitation to random groups}




\section{Models of typical groups}

The basic idea of random groups is to take a group presentation at random and to look at what are "typically" the properties of the group so obtained, leading to such statements as "almost every group is hyperbolic". Of course this makes sense only if some precise way to pick presentations at random is prescribed: this is what we call a model of random groups.

So a random group will usually be given by a presentation by generators and relators

$$
G=\langle S \mid R\rangle
$$

where $S=\left\{a_{1}, \ldots, a_{m}\right\}$ is some finite ${ }^{1}$ set of generators, and $R$ is a set of words on the elements of $S$ (and their inverses), taken at random. Since any group presentation can be written using reduced words only (i.e. words not containing $a_{i} a_{i}^{-1}$ or $a_{i}^{-1} a_{i}$ ), usually only such words are considered.

To choose a model of random groups is to specify a probability law for the set of relators $R$. Probability and statistics are most relevant when some parameter is large so that laws of large numbers can be used. In most (but not all) models, the set of generators $S=\left\{a_{1}, \ldots, a_{m}\right\}$ is kept fixed, and the large parameter is the length of the words in $R$. One more degree of freedom is to let the number of words in $R$ grow as their length becomes larger. These choices allow for different models.

The models. Basically there are three models of random groups. The quite straightforward few-relator model (Def. 1) allows for only a fixed number of relators, of bounded length; small cancellation, hence hyperbolicity, is easily shown to be generic in this model. It is now subsumed as density 0 in the density model. The few-relator model with various lengths (Def. 4), which allows very different relator lengths, is more difficult technically because several scales are involved. The density model (Def. 7) allows a clear-cut quantitative approach on the number of relators that can be put before the group collapses; this model has been preferentially focussed on recently because various values of the density parameter involved seem to have different, rather concrete geometrical meanings. One variant of the density model is the triangular model (§ I.3.g.), which is somehow "less quotiented" and often produces only free groups.

\footnotetext{
${ }^{1}$ It must be stressed that although any group has some presentation (as described in the Primer), group presentations are mainly relevant for countable groups only, and the geometric methods work best for finitely presented groups. Thus the models of random groups currently used focus on those.
} 
All of these models lead to the same conclusion that a typical (finitely presented) group is hyperbolic. (For hyperbolic groups we refer the reader to the Primer or to [BH99, Sho91a, GhH90, Ghy90, CDP90, Gro87].)

The topological approach of the space of marked groups (§ I.4.), though not a model of random groups itself, may be a nice framework to interpret some of these results in.

Another would-be model, arguably the most natural of all, the temperature model, is kind of a density model at all relator lengths simultaneously, thus producing non-finitely presented groups. It is addressed as an open question in $\S$ IV.k. since there still are no results about it.

Partially random groups. There has been some confusion due to the fact that the most famous (up to date) "random groups", those constructed by Gromov having no uniform embedding into the Hilbert space, exhibit quite different properties from what is hereafter described as typical for a random group (e.g. they are not hyperbolic). Actually the construction of these groups (thoroughly discussed in $\S$ III.) involves both random ingredients and manipulations quite specific to the goal of controlling uniform embeddings, and they are thus rather non-typical; dubbing them "partially random" would be more appropriate.

\section{I.1. Forerunners: few-relator models}

The statement that most groups are hyperbolic is statistical. It means that out of all possible group presentations, asymptotically most of them define hyperbolic groups. Here the asymptotics are taken with respect to the length of the relators involved.

Maybe the simplest statement expressing the overwhelming weight of hyperbolic presentations consists in considering the set of all presentations with a fixed number of relators and a bounded relator length, as in the following model.

DEFinition 1 (FEW-RELATOR MODEl OF RANDOM GROUPS) Let $\mathcal{R}_{k, \ell}$ be the set of all group presentations with $k$ relators of length at most $\ell$

$$
\mathcal{R}_{k, \ell}=\left\{\left\langle a_{1}, \ldots, a_{m} \mid r_{1}, \ldots, r_{k}\right\rangle, r_{i} \text { reduced, }\left|r_{i}\right| \leqslant \ell \quad \forall i\right\}
$$

Let $P$ be a property of a presentation. We say that $P$ occurs with overwhelming probability in this model if the share of presentations in $\mathcal{R}_{k, \ell}$ which have property $P$ tends to 1 as $\ell$ tends to infinity.

The following proposition was more or less implicit in the original formulation of small cancellation theory. Let us simply recall that $C^{\prime}(\lambda)$ for $\lambda>0$ is the condition that no two relators in a presentation share a common subword of length at least $\lambda$ times the infimum of their lengths (we 
refer to [LS77] for small cancellation theory). When $\lambda<1 / 6$ this implies hyperbolicity ([Gro87], 0.2.A).

Proposition 2 - For any $k$, for any $\lambda>0$, the $C^{\prime}(\lambda)$ small cancellation property occurs with overwhelming probability in the few-relator model of random groups. In particular, hyperbolicity occurs with overwhelming probability in this model, as well as torsion-freeness and cohomological dimension 2.

Of course, the overwhelming probability depends on $k$ and $\lambda$ : for very small $\lambda$ 's, it is necessary to take larger $\ell$ for the share to become close to 1 .

Note also that since the number of possible relators of length $\ell$ grows exponentially with $\ell$, a random relator of length at most $\ell$ actually has length between $\ell(1-\varepsilon)$ and $\ell$, so that in this model all relators have almost the same length.

This proposition appears in [Gro87], 0.2.A (in the notation thereof, this is the case when $\ell_{2} / \ell_{1}$ is very close to 1 ). This is the model referred to as "généricité faible" (weak genericity) in [Ch91, Ch95]. The proof is straightforward. Take e.g. $k=2$. The number of couples of reduced relators of length at most $\ell$ behaves like $(2 m-1)^{2 \ell}$, whereas the number of couples of relators sharing a common subword of length $\lambda \ell$ behaves roughly like $(2 m-1)^{2 \ell-\lambda \ell}$. So the share of couples of relators having a common subword of length $\lambda \ell$, for some $\lambda>0$, is exponentially small when $\ell \rightarrow \infty$, so that the $C^{\prime}(\lambda)$ condition is satisfied (a little more care is needed to treat the case of a piece between two parts of the same relator).

REMARK 3 - The few-relator model of random groups appears as the 0 -density case of the density model.

For this reason, results known to hold in this model are discussed below in $\S \mathrm{I} .2$.

In [Gro87], 0.2.A, Gromov immediately notes that it is not necessary to assume that all relators have lengths of the same order of magnitude to get hyperbolicity. This yields to the next model, which is technically much more difficult.

DeFinition 4 (FEW-RELATOR MOdel With VARIOUS LeNGTHS) Given $k$ integers $\ell_{1}, \ldots, \ell_{k}$, let

$$
\mathcal{R}_{k, \ell_{1}, \ldots, \ell_{k}}=\left\{\left\langle a_{1}, \ldots, a_{m} \mid r_{1}, \ldots, r_{k}\right\rangle, \quad r_{i} \text { reduced, }\left|r_{i}\right|=\ell_{i}\right\}
$$

be the set of presentations with $k$ relators of prescribed lengths.

Let $P$ be a property of a presentation. We say that $P$ occurs with overwhelming probability in this model if for any $\varepsilon>0$ there exists an $\ell$ such that, if $\min \ell_{i} \geqslant \ell$, then the share of presentations in $\mathcal{R}_{k, \ell_{1}, \ldots, \ell_{k}}$ which have property $P$ is greater than $1-\varepsilon$. 
In general, the small cancellation condition $C^{\prime}(\lambda)$ is not satisfied in this model. Indeed, as soon as e.g. $\ell_{2}$ is exponentially larger than $\ell_{1}$, very probably the relator $r_{1}$ will appear as a subword of the relator $r_{2}$.

Once again however, hyperbolicity occurs with overwhelming probability. This is stated without proof in [Gro87], 0.2.A, and is referred to as "Théorème sans preuve" in [GhH90]. A little bit later, proofs were given independently by Champetier [Ch91, Ch95] (in the case $k=2$ ) and Ol'shanskiǐ [Ols92].

Theorem 5 - With overwhelming probability, a random group in the few-relator model with various lengths is non-elementary hyperbolic, torsionfree, of cohomological dimension at most 2.

A few more results are known in this model. For $k=2$, the boundary of the group is a Menger curve [Ch95] (see also § I.3.d.). Also the rank is the one expected [CS98] (and in particular the cohomological dimension in the previous theorem is actually 2 ):

Theorem 6 - With overwhelming probability, a random group in the few-relator model with various lengths has the following property: the subgroup generated by any $m-1$ generators chosen among $a_{1}, \ldots, a_{m}$ is free of rank $m-1$.

Moreover, thanks to a theorem of Champetier [Ch93] the spectral radius of the random walk operator associated with $a_{1}, \ldots, a_{m}$ (see definition in $\S$ I.3.f.) is arbitrarily close to the smallest possible value $\sqrt{2 m-1} / m$ [CS98]. Using similar spectral bounds, it is proven in [CV96] that for $k=1$ (one relator), the semi-group generated by $a_{1}, \ldots, a_{m}$ is free.

Let us stress that contrary to the few-relator, one-length model, the fewrelator model with various lengths is not subsumed in the density model below. It might, however, be recovered as an iterated random quotient at density 0 (see $\S$ II.2.), but the technical details needed to get this are still unclear.

\section{I.2. Gromov's density}

I.2.a. Definition of density. By the time Champetier and Ol'shanskir had proven his first statement, Gromov had already invented another model, the density model ([Gro93], Chapter 9 entitled Finitely presented groups: density of random groups and other speculations). A continuous density parameter now controls the quantity of relators put in the random presentation. The sharpness of the notion is revealed through a phase transition theorem: if density is less than $1 / 2$, then the random group is very probably infinite hyperbolic, whereas it is trivial at densities above $1 / 2$. 


\section{Definition 7 (Density model of RANDom groups) -}

Let $F_{m}$ be the free group on $m \geqslant 2$ generators $a_{1}, \ldots, a_{m}$. For any integer $\ell$ let $S_{\ell} \subset F_{m}$ be the set of reduced words of length $\ell$ in these generators.

Let $0 \leqslant d \leqslant 1$. A random set of relators at density $d$, at length $\ell$ is a $(2 m-1)^{d \ell}$-tuple of elements of $S_{\ell}$, randomly picked among all elements of $S_{\ell}$ (uniformly and independently).

$A$ random group at density $d$, at length $\ell$ is the group $G$ presented by $\left\langle a_{1}, \ldots, a_{m} \mid R\right\rangle$ where $R$ is a random set of relators at density $d$, at length $\ell$.

We say that a property of $R$, or of $G$, occurs with overwhelming probability at density $d$ if its probability of occurrence tends to 1 as $\ell \rightarrow \infty$, for fixed $d$.

REMARK 8 - Slight variants of this historical definition exist, sometimes leading to more nicely expressed statements, e.g. replacing the sphere $S_{\ell}$ with the ball $B_{\ell}$ of words of length at most $\ell$. They are discussed in $\S$ I.2.c.

Of course, the main point in this definition is the number $(2 m-1)^{d \ell}$ of relators taken, which is actually quite large. Note that the set $S_{\ell}$ contains about $(2 m-1)^{\ell}$ words, so that density is measured logarithmically (a fact we will meet again in $\S$ II.). The intuition behind this and the strong analogy with usual dimension and intersection theory are very nicely explained in [Gro93] (see also [Ghy03]): for a finite set $X$, the density of $A \subset X$ defined as $d(A)=\log \# A / \log \# X$ has, for "generic" $A$, lots of expected properties of a dimension (e.g. for the dimension of an intersection).

The basic idea is that $d \ell$ is the "dimension" of the random set of relators $R$ (the set $S_{\ell}$ itself being considered of dimension $\ell$ because we have $\ell$ independent letter choices to make to specify an element in $S_{\ell}$ ).

Classically, the dimension of a set (subspace in a vector space, algebraic submanifold) is the maximal number of "independent equations" that we can impose so that there still exists an element in the set satisfying them. For words, an "equation" will mean prescribing some letter in the word. Now consider e.g. a set of $2^{d \ell}$ random words of length $\ell$ in the two letters $a$ and $b$; a simple counting argument shows that very probably, one of these words will begin with roughly $d \ell$ letters $a$ (but not much more), meaning that this random set has "dimension" $d \ell$. More precisely:

Proposition 9 - Let $R$ be a random set of relators at density $d$, at length $\ell$. Let $0 \leqslant \alpha<d$. Then with overwhelming probability the following occurs: Any reduced word of length $\alpha \ell$ appears as a subword of some word in $R$.

Note that by a trivial cardinality argument, if $\alpha>d$ there exists a reduced word of length $\alpha \ell$ not appearing as a subword of any word in $R$.

Let us show on another example the strength (and correctness) of dimensional reasoning: Let us compute the probability that there exist two relators in $R$ sharing a common subword of length $\alpha \ell$. The dimension of 
$R$ is $d \ell$, so that the dimension of the set of couples $R \times R$ is $2 d \ell$. Now sharing a common subword of length $L$ imposes $L$ equations, so that the "dimension" of the set of couples of relators sharing a common subword of length $\alpha \ell$ is $2 d \ell-\alpha \ell$. So if $d<\alpha / 2$ this dimension will tend to $-\infty$ as $\ell \rightarrow \infty$, implying that there will be no such couple of relators; conversely if $d>\alpha / 2$ there will exist such a couple because dimension will be positive. What we have "shown" is:

Proposition $10-$ Let $\alpha>0$ and $d<\alpha / 2$. Then with overwhelming probability, a random set of relators at density $d$ satisfies the $C^{\prime}(\alpha)$ small cancellation condition.

Conversely, if $d>\alpha / 2$, then with overwhelming probability a random set of relators at density $d$ does not satisfy the $C^{\prime}(\alpha)$ small cancellation condition.

A rigorous proof ([Gro93], 9.B) is obtained by a simple counting argument, which in fact amounts to raising $(2 m-1)$ to the exponents given by the various "dimensions" of the sets involved.

I.2.b. The phase transition. The striking phase transition theorem then proven by Gromov in [Gro93] is as follows.

Theorem 11 - Let $G$ be a random group at density $d$.

- If $d<1 / 2$, then with overwhelming probability $G$ is infinite, hyperbolic, torsion-free, of geometric dimension 2.

- If $d>1 / 2$, then with overwhelming probability $G$ is either $\{e\}$ or $\mathbb{Z} / 2 \mathbb{Z}$.

We include a proof of this theorem in $\S \mathrm{V}$. The argument appears in [Gro93], pp. 273-275; it suffers from omission of the case when a van Kampen diagram comprises the same relator several times. The proof of a similar-looking statement (Theorem 29 in the triangular model, see $\S$ I.3.g.) in [Żuk03] suffers from a similar but more subtle flaw (see $\S$ V.). A somewhat lengthy proof is given in [Oll04].

This calls for a few comments: Of course $\mathbb{Z} / 2 \mathbb{Z}$ occurs for even $\ell$. What happens at exactly $d=1 / 2$ is unknown and even the right way of asking the question is unclear (see $\S$ IV.a. for an elaboration on this). Note that Proposition 10 already implies the conclusion for $d<1 / 12$, for then the presentation satisfies the good old $C^{\prime}(1 / 6)$ small cancellation condition.

This theorem generalizes to random quotients of torsion-free hyperbolic groups (§ II.1. and $\S$ II.2.).

The reason for density $1 / 2$ is the following: Recall the probabilistic pigeon-hole principle ${ }^{2}$, which states that if in $N$ holes we put much more

\footnotetext{
${ }^{2}$ A.k.a. the birthday paradox: in a class of more than 23 pupils there is a good chance that two of them share the same birthday. This is a simple combinatorial exercise.
} 
than $\sqrt{N}$ pigeons then we will put two pigeons in the same hole (very probably as $N \rightarrow \infty$, provided that the assignment was made at random). In other words, a generic set of density more than $1 / 2$ does self-intersect.

Density $1 / 2$ is the case when the cardinal of the set of relators $R$ is more than the square root of the cardinal of the set $S_{\ell}$ of words of length $\ell$. In particular, this means that we very probably picked twice the same relator in $R$. A fortiori, very probably there are two relators $r_{1}, r_{2} \in R$ differing just at one position i.e. $r_{1}=w a_{i}^{ \pm 1}, r_{2}=w a_{j}^{ \pm 1}$ with $|w|=\ell-1$. But in the group $G=F_{m} /\langle R\rangle$, we have by definition $r_{1}={ }_{G} r_{2}={ }_{G} e$, so that $a_{i}^{ \pm 1}={ }_{G} a_{j}^{ \pm 1}$. Since there are only a finite number of generators, this will eventually occur for every value of $i$ and $j$ and every sign of the exponent, so that in $G$ any generator will be equal to any other and to its inverse, implying that the group has only one or two elements.

This proves the trivial part of the theorem.

I.2.c. Variations on the model. Several points in the definition above are left for interpretation. First, let us stress that it is not crucial to take relators of length exactly $\ell$ : choosing lengths between $\ell$ and $\ell+o(\ell)$ would do as well. This even has several advantages: it kills the odd $\mathbb{Z} / 2 \mathbb{Z}$ in Theorem 11 and avoids matters of divisibility by 3 in the property $(T)$ theorem (Theorem 27).

Actually the most natural setting is perhaps to choose at random words of length at most $\ell$ ("ball variant") instead of exactly $\ell$. Since the number of words grows exponentially, most words so taken will be of length close to $\ell$, but since the number of words taken is exponential too, some words will be shorter (at density $d$ the shortest word will have length approximately $(1-d) \ell)$. This variant simplifies the statements of Theorems 11, 27 and 38, is more natural for random quotients ( $§$ II.), and the validity of all random group theorems proven so far seems to be preserved. In this text we chose to keep the historical Definition 7, in order to quote the literature without change; but e.g. for a textbook on random groups, the ball variant might be preferable.

There is a slight difference between choosing $N$ times a random word and having a random set of $N$ words, since some word could be chosen several times. But for $d<1 / 2$ the probability that a word is chosen twice is very small and the difference is negligible; anyway this does not affect our statements, so both interpretations are valid.

Numbers such as $(2 m-1)^{d \ell}$ are not necessarily integers. We can either take the integer part, or choose two constants $C_{1}$ and $C_{2}$ and consider taking any number of words between $C_{1}(2 m-1)^{d \ell}$ and $C_{2}(2 m-1)^{d \ell}$. Once more this does not affect our statements at all.

One may hesitate between choosing reduced or cyclically reduced words. Once again this does not matter.

Section 4 of [Oll04] (in particular Remark 8) contains an axiomatic framework which allows to handle such a loose model and not to reprove all the 
theorems for each variant.

In all theorems stated in this text, not only does "with overwhelming probability" mean that the share of groups not having the property under consideration tends to 0 as $\ell \rightarrow \infty$, but actually the decay is exponential, that is, there exists a constant $c$ (depending on everything except $\ell$ ) such that this share is less than $\exp (-c \ell)$.

A very natural generalization of the density model is the temperature model, described in § IV.k.

REMARK 12 (ON DENSITY 0) - The intuition makes it clear that the only thing that matters is the exponent of growth of the number of relators. Thus, although it would follow from Definition 7 that a random set of relators at density 0 consists of one relator, we often use "density 0 " to refer to a situation when the number of relators grows subexponentially with their lengths, e.g. the case of a constant number of relators (the fewrelator model of Def. 1-but not the one of Def. 4).

\section{I.3. Critical densities for various properties}

A bunch of properties are now known to hold for random groups. This ranges from group combinatorics (small cancellation properties) to algebra (freeness of subgroups) to geometry (boundary at infinity, growth exponent, CAT(0)-ness) to probability (random walk in the group) to representation theory on the Hilbert space (property $(T)$, Haagerup property). Some of the properties studied here are intrinsic to the group, others depend on a marked set of generators or on the standard presentation through which the random group was obtained.

Most interesting is the fact that some intrinsic properties vary with density (property $(T)$, Haagerup property), thus proving that different densities can provide non-isomorphic groups (see $\S$ IV.b. for a discussion of this problem).

I.3.a. Van Kampen diagrams and small cancellation properties. These are the most immediate properties one gets for a random group. They are properties of the presentation, not of the abstract group.

Hyperbolicity of random groups is proven through isoperimetry of van Kampen diagrams (see the Primer for what we need on van Kampen diagrams or [LS77, Ols91a, Rot95] for definitions and [Sho91a] for the link with hyperbolicity). Various, closely related formulations of this inequality for random groups appear in [Gro93, Ch91, Ols92, Ch95, Ollo4, Oll-f]. We give the most recent one from [Oll-f], which is sharp and, combined with a result in [Ch94], gives a nice estimate for the hyperbolicity constant:

Theorem 13 - For every $\varepsilon>0$, with overwhelming probability, every reduced van Kampen diagram $D$ in a random group at density $d<1 / 2$, at 
length $\ell$ satisfies

$$
|\partial D| \geqslant(1-2 d-\varepsilon) \ell|D|
$$

where $|\partial D|$ is the boundary length and $|D|$ the number of faces of $D$.

Consequently, the hyperbolicity constant $\delta$ of the random group satisfies

$$
\delta \leqslant \frac{4 \ell}{1-2 d}
$$

and the length of the smallest relation in the group is at least $\ell(1-2 d-\varepsilon)$.

These properties are understood with respect to the standard presentation from which the random group was obtained.

This theorem of course implies Theorem 11. The complete argument is given in $\S \mathrm{V}$.

The isoperimetric constant is optimal in the sense that, with overwhelming probability, there exists a two-face van Kampen diagram $D$ satisfying $|\partial D| \leqslant(1-2 d+\varepsilon) \ell|D|$, which is just the failure of the $C^{\prime}(2 d+\varepsilon)$ small cancellation property (Prop. 10). For the hyperbolicity constant, clearly $\ell$ is the right order of magnitude but the real dependency on $d$ when $d \rightarrow 1 / 2$ is unclear.

Next come some small cancellation conditions. By the way, actually as $d$ approaches $1 / 2$, we have arbitrarily large cancellation (which refutes the expression "small cancellation on average" sometimes applied to this theory - we indeed measure cancellation on average, but it is not small), as results from the next proposition.

Recall that, given a group presentation, a piece is a word which appears as a subword of two different relators in the presentation, or as a subword at two different positions in the same relator (relators are considered as cyclic words and up to inversion). For $\alpha>0$, the most often used $C^{\prime}(\alpha)$ condition states that the length of any piece is less than $\alpha$ times the infimum of the lengths of the relators on which it appears. For an integer $p$, the $C(p)$ condition holds if no relator is the union of less than $p$ pieces. The $B(2 p)$ condition holds if the union of $p$ consecutive pieces always makes less than half a relator. We have the implications $C^{\prime}(1 / 2 p) \Rightarrow B(2 p) \Rightarrow C(2 p+1)$. The $T(p)$ small cancellation condition is totally irrelevant for groups with lots of relators.

Conditions $C^{\prime}(1 / 6), C(7)$ or $B(6)$ imply hyperbolicity. Elementary counting arguments [OW-b] yield the following more erudite version of Proposition 10:

Proposition 14 - With overwhelming probability:

- The $C^{\prime}(\alpha)$ condition occurs if $d<\alpha / 2$ and fails for $d>\alpha / 2$,

- The $C(p)$ condition occurs if $d<1 / p$ and fails for $d>1 / p$,

- The $B(2 p)$ condition occurs if $d<1 /(2 p+2)$ and fails for $d>1 /(2 p+$ 2). 
These conditions being understood for the standard presentation from which the random group was obtained.

In particular, using the $C(7)$ condition, this proposition proves Theorem 11 up to density $1 / 7$.

Closely related to small cancellation are Dehn's algorithm (see [LS77]), which holds for a group presentation when every reduced cyclic word representing the identity in the group has a subword which is more than half a subword of a relator in the presentation; and its stronger version, Greendlinger's Lemma (see [LS77] too), which holds when every reduced van Kampen diagram with at least two faces has at least two faces having more than half their length on the boundary of the diagram (in one piece). Every hyperbolic group admits some finite presentation satisfying Dehn's algorithm ([Sho91a], Theorem 2.12). However, for the standard presentation of a random group, a phase transition occurs at $1 / 5$ [Oll-f]:

Theorem 15 - With overwhelming probability, if $d<1 / 5$ the standard presentation of a random group at density $d$ satisfies Dehn's algorithm and Greendlinger's Lemma. If $d>1 / 5$, with overwhelming probability it does not satisfy any of the two.

This property is the last remnant of combinatorial small cancellation when density increases. It is crucial in the proof of Theorems 32 and 33 about action on a cube complex and failure of property $(T)$.

I.3.b. Dimension of the group. A consequence of the isoperimetric inequality holding for any reduced van Kampen diagram is that the Cayley 2-complex associated with the presentation is aspherical [Gro93, Oll04], so that the group has geometric (hence cohomological) dimension 2 as stated in Theorem 11. The Euler characteristic of the group is thus simply $1-m+(2 m-1)^{d \ell}$.

In particular, since this Euler characteristic is positive for large $\ell$, we get the following quite expected property (at least for $d>0$, but this also holds at density 0 thanks to Theorem 18):

Proposition 16 - With overwhelming probability, a random group in the density model is not free.

Consideration of the Euler characteristic also implies that, for fixed $m$, the "dimension" $d \ell$ of the set of relations of the group is well-defined by its algebraic structure.

I.3.c. Algebraic properties at density 0: rank, free subgroups. When density is 0 (i.e. in the few-relator model, see Def. 1 and Remark 12), random groups keep lots of algebraic properties of a free group. In a certain sense, there are "no more" relations holding in the group than those 
explicitly put in the presentation. Several theorems in this direction are proven by Arzhantseva and Ol'shanskiı̌, using a technique of representation of subgroups of a group by labelled graphs (or finite automata) introduced by Stallings [Sta83], technique which will be discussed more in $\S$ III.1. Arzhantseva and Ol'shanskir are able to extract, from failure of freeness in subgroups of a random group, a "too small" representation of one of the relators, which never occurs for random relators.

It is clear that some (all?) of these theorems do not hold at all densities. But they probably extend to small positive values of $d$, the determination of which is an interesting problem.

The first such theorem [AO96] is the following:

Theorem 17 - With overwhelming probability in the few-relator model of random groups with $m$ generators and $n$ relators, any subgroup generated by $m-1$ elements is free.

This is not true at all densities (see $\S$ IV.d.). When the $m-1$ generators of the subgroup are chosen among the $m$ standard generators of the group, this is a particular case of Theorem 6 .

The group itself is not free and more precisely [Ar97]:

Theorem 18 - In the few-relator model of random groups with $n \geqslant 1$ relators, no finite-index subgroup of the group is free.

As a corollary of these two theorems, we see that the rank of the random group in the few-relator model is exactly $m$, which, once again, does not hold at all densities (cf. § IV.d.).

Reusing the methods of Arzhantseva and Ol'shanskiĭ, Kapovich and Schupp prove that there is "only one" $m$-tuple generating the group. Recall [LS77] that for a $m$-tuple of elements $\left(g_{1}, \ldots, g_{m}\right)$ in a group, a Nielsen move consists in replacing some $g_{i}$ with its inverse, or interchanging two $g_{i}$ 's, or replacing some $g_{i}$ with $g_{i} g_{j}$ for some $i \neq j$. Obviously these moves do not change the subgroup generated by the $m$-tuple. The theorem [KS05] reads:

Theorem 19 - With overwhelming probability, in a random few-relator group $G$, any $m$-tuple of elements generating a non-free subgroup is Nielsenequivalent in $G$ to the standard $m$-tuple of generators w.r.t. which the random presentation was taken.

In particular, any automorphism of $G$ lifts to an automorphism of $F_{m}$.

More properties of free groups are kept by random few-relator groups. In a free group, any subgroup is free; any finitely generated subgroup is quasiconvex; any non-trivial finitely generated normal subgroup has finite index ([LS77], Prop. I.3.12); the intersection of any two finitely generated subgroups is finitely generated (Howson's Theorem, [LS77], Prop. I.3.13). These properties are generalized as follows in [Ar97, Ar98]: 
Theorem 20 - Let $L \geqslant 1$ be an integer. With overwhelming probability, a random few-relator group satisfies the following properties.

- Any subgroup of rank at most $L$ and of infinite index is free.

- Any subgroup of rank at most $L$ is quasiconvex.

- Any non-trivial normal subgroup of rank at most $L$ has finite index.

- The intersection of any two subgroups of rank at most $L$ is quasiconvex and finitely generated.

The overwhelming probability depends on $L$. For example, it is not clear whether all infinite-index subgroups are free or not.

The last point follows from the second one, noting that, in a finitely generated group, the intersection of two quasiconvex subgroups is quasiconvex and quasiconvex subgroups are finitely generated (see the nice [Sho91b]).

The results mentioned so far deal with properties of subgroups in the random group. One can wonder how subgroups of the free group are mapped to the random group. A theorem in this direction is the following [Ar00]:

Theorem 21 - Let $h_{1}, \ldots, h_{k}$ be elements of the free group $F_{m}$ generating a subgroup $H$ of infinite index. Then with overwhelming probability, the map from $F_{m}$ to a random few-relator group is injective on $H$.

Conversely, it is easily seen that subgroups of finite index do not embed.

Of course this holds for elements $h_{1}, \ldots, h_{k}$ fixed in advance: it cannot be true that the quotient map is injective on all subgroups...

I.3.d. Boundary and geometric properties of the Cayley graph. We refer to [GhH90, CDP90, BH99] for the notion of boundary of a hyperbolic space.

Since the dimension of a random group is 2 (§ I.3.b.), by Corollary 1.4 of [BM91], the dimension of its boundary is 1. Champetier ([Ch95], Theorem 4.18) proves that at small density, the boundary is the most general object of dimension 1 :

Theorem 22 - Let $d<1 / 24$. Then with overwhelming probability, the boundary of a random group is a Menger curve. In particular the group is one-ended.

The bound $1 / 24$ is probably not sharp. Let us recall that the Menger curve is the universal object in the category of compact metric spaces of dimension 1, see [And58]; it is (almost) characterized as the 1-dimensional, locally connected, locally non-planar continuum without local cut points (the boundary of a one-ended hyperbolic group never has cut points [Swa96]).

One-endedness probably holds at any density, but between $1 / 24$ and $1 / 3$ no simple criterion seems to apply. For $d>1 / 3$, using Serre's theory of groups acting on trees [Ser77, HV89] it is a corollary of Theorem 27 on property $(T)$ : 
Proposition $23-$ Let $d>1 / 3$. Then with overwhelming probability, a random group is one-ended.

At density 0, the Cayley graph of the group is not planar [AC04] (planarity of Cayley graph and complexes is an old story, see discussion in [AC04]). The result actually holds for generic $C^{\prime}(1 / 8)$ small cancellation groups and so:

Theorem $24-$ Let $d<1 / 16$. With overwhelming probability, the Cayley graph (w.r.t. the standard generating set) of a random group at density $d$ is not planar.

Actually the technique used in [AC04] allows to embed subdivisions of lots of finite graphs into the Cayley graph of a small-density random group.

I.3.e. Growth exponent. The growth exponent of a group presentation $G=\left\langle a_{1}, \ldots, a_{m} \mid R\right\rangle$ measures the rate of growth of balls in the group. Let $B_{L}$ be the set of elements of the group $G$ which can be written as a word of length at most $L$ in the generators $a_{1}^{ \pm 1}, \ldots, a_{m}^{ \pm 1}$. If $G$ is the free group $F_{m}$, the number of elements of $B_{L}$ is $1+\sum_{k=1}^{L}(2 m)(2 m-1)^{L-1}$ which is the number of elements at distance at most $L$ from the origin in the valency- $2 m$ regular tree. The thing that matters here is the exponential growth rate of the balls,

$$
g=\lim _{L \rightarrow \infty} \frac{1}{L} \log _{2 m-1} \# B_{L}
$$

(the limit exists thanks to the relation $\# B_{L+L^{\prime}} \leqslant \# B_{L} \# B_{L^{\prime}}$ ). This quantity is the growth exponent of the group $G$ w.r.t. the generating set $a_{1}, \ldots, a_{m}$. It is at most 1 , and equal to 1 if and only if $G$ is the free group $F_{m}$ on these generators. See [Har00] (chapters VI and VII), [GH97] or [Ver00] for some surveys and applications related to growth of groups.

Actually, the growth exponent of a random group at any density $d<$ $1 / 2$ is arbitrarily close to that of a free group with the same number of generators [Oll-b]. Of course, by Theorem 13 a random group behaves like a free group up to scales $\ell(1-2 d)$, but growth is an asymptotic invariant taking into account the non-trivial geometry of the group at scale $\ell$, so it is somewhat surprising that the growth exponent is large independently of the density $d$ (except if $d>1 / 2$ where of course it drops to 0 ). Computing the growth exponent was initially an attempt to build a continuous quantity depending on density.

Theorem $25-$ Let $\varepsilon>0$ and $0 \leqslant d<1 / 2$. Then with overwhelming probability, the growth exponent of a random group at density $d$ lies in the interval $[1-\varepsilon ; 1)$ (w.r.t. the standard generating set).

Note that non-sharp bounds for the growth exponent can be obtained from the spectral estimates discussed in $§$ I.3.f. (see discussion in [Oll-b]). 
When $d<1 / 12$ the random group satisfies the $C^{\prime}(1 / 6)$ small cancellation condition, and in this case this result is related to a theorem of Shukhov [Shu99] stating that $C^{\prime}(1 / 6)$ groups with long enough relators and "not too many" relators have growth exponent close to 1 . Shukhov's "not too many" relators condition is strikingly reminiscent of a density condition.

I.3.f. Random walk in a typical group. A group together with a generating set defines a random walk, which consists in starting at $e$ and, at each step, multiplying by one of the generators or its inverse, chosen at random (this is the simple random walk on the Cayley graph). A foundational paper of this theory is that of Kesten [Kes59], see also [Gri80, GH97, Woe00] for some reviews.

One quantity containing a lot of information about the random walk is the spectral radius of the random walk operator (see [Kes59]). Let $P_{t}$ be the probability that at time $t$, the random walk starting at $e$ is back at $e$. The spectral radius of the random walk is defined as

$$
\rho=\lim _{\substack{t \rightarrow \infty \\ t \text { even }}}\left(P_{t}\right)^{1 / t}
$$

(the limit exists thanks to the property $P_{t+t^{\prime}} \geqslant P_{t} P_{t^{\prime}}$ ). One restricts oneself to even $t$ because there might be no odd-length path from $e$ to $e$ in the Cayley graph. This quantity is at most 1 (a value achieved if and only if the group is amenable [Kes59]), and at least $\frac{\sqrt{2 m-1}}{m}$ where $m$ is the number of generators (achieved if and only if the group is free on these generators).

Just as for the growth exponent, it came out as a surprising fact that the spectral radius of the random walk on a random group does not depend on density [Oll05a], except of course when $d>1 / 2$ where it suddenly jumps to 1 . Once again this cannot be interpreted simply by saying that random groups are free up to scale $\ell(1-2 d)$, because the spectral radius is an asymptotic invariant taking into account the non-trivial geometry at scale $\ell$.

Theorem $26-$ Let $\varepsilon>0$ and $0 \leqslant d<1 / 2$. Let $\rho\left(F_{m}\right)=\frac{\sqrt{2 m-1}}{m}$ be the spectral radius of the random walk on the free group $F_{m}$.

Then with overwhelming probability, the spectral radius of the random walk on a random group at density $d$ lies in the interval $\left(\rho\left(F_{m}\right) ; \rho\left(F_{m}\right)+\varepsilon\right)$.

At density 0 this follows from a theorem of Champetier [Ch93], which, as mentioned earlier (§ I.1.), also holds in the few-relator model with various lengths.

Consequently, the growth exponent of the kernel of the map $F_{m} \rightarrow G$ (the cogrowth exponent of $G$ ) is less than $1 / 2+\varepsilon$, thanks to a formula by Grigorchuk ([Gri80, Ch93, Oll04]). This answers Gromov's question 9.B.(c) 
in [Gro93]: normal closures of random sets of density $<1 / 2$ generated by random large elements in $F_{m}$ have "density" (growth exponent) less than $1 / 2+\varepsilon$.

This result also has a nice interpretation in terms of a random walk on an infinite tree with lots of "zero-length" bridges added at random (in an "equivariant" way). Indeed the random walk on a random group $G$ can be thought of as a random walk on the Cayley graph of the free group $F_{m}$ where elements of $F_{m}$ mapping to the same element of $G$ are linked by a bridge through which the random walk can "instantly" travel. The theorem then states that adding "lots of" bridges equivariantly does not change the probability to come back to (a point connected by bridges to) the origin, up till some density when suddenly any point is connected to the origin by a sequence of bridges.

I.3.g. Property $(T)$ and the triangular model. Kazhdan's property $(T)$ of a group has to do with the behavior of unitary actions of the group on the Hilbert space and basically asks that, if there are unitary vectors which the group action moves by arbitrarily small amounts, then there is a vector fixed by the action. It has proven to be linked with numerous algebraic or geometric properties of the group. We refer to [HV89, BHV, Val02a] for reviews and basic properties.

The so-called spectral criterion is a sufficient condition for property $(T)$ of a discrete group, which is an explicitly checkable property of the ball of radius 1 in the Cayley graph w.r.t. some generating set. The neatest statement is to be found in [Żuk03], see also [Z̈uk96, BŚ97, Pan98, Wan98, Val02a]. Gromov (part 3 of [Gro03]) put this result in a more general context, which allowed Ghys to write a very simple proof [Ghy03, Oll-d].

It happens that in the density model, after suitable manipulations of the presentation, this criterion is satisfied as soon as $d>1 / 3$. It is not known whether this latter value is optimal (compare Theorem 32 below and $\S$ IV.c.).

Theorem 27 - Let $d>1 / 3$ and let $G$ be a random group at density $d$ and at lengths $\ell, \ell+1$ and $\ell+2$. Then, with overwhelming probability, $G$ has property $(T)$.

The necessity to take a random quotient at three lengths simultaneously is a technical annoyance due to the too restrictive definition of the density model, which disappears if we replace the sphere by the ball in Definition 7 (see Remark 8 and $\S$ I.2.c.). This results from the necessity to have some relators of length a multiple of 3 , as we explain now.

This theorem is proven using an intermediate random group model better suited to apply the spectral criterion, the triangular model, which we now define. This model consists in taking relators of length only 3 , but letting the number of distinct generators tend to infinity. Żuk [Żuk03] wrote 
a proof that property $(T)$ holds in this model at density $d>1 / 3$ (Theorem 31 ); it is then possible to carry the result to the density model (but actually Theorem 27 seems not to be written anywhere explicitly in the literature).

Definition 28 (Triangular MOdel) - Let $n$ be an integer and let $b_{1}, \ldots, b_{n}$ be $n$ distinct symbols. Let $W_{n, 3}$ be the set of words of length 3 in $b_{1}, \ldots, b_{n}, b_{1}^{ \pm 1}, \ldots, b_{n}^{ \pm 1}$ which are cyclically reduced.

Let $0 \leqslant d \leqslant 1$. A random group on $n$ relators at density $d$ in the triangular model is the group presented by $G=\left\langle b_{1}, \ldots, b_{n} \mid R_{3}\right\rangle$ where $R_{3}$ is a set of $\left(\# W_{n, 3}\right)^{d}$ words taken at random in $W_{n, 3}$.

$A$ property of $G$ is said to occur with overwhelming probability in this model if its probability of occurrence tends to 1 as $n \rightarrow \infty$.

Note that $\# W_{n, 3} \sim(2 n)^{3}$ for large $n$. The density intuition is the same as above: the number of relators taken is a power of the total number of possibilities.

Actually there is a natural homomorphism from a random group in the triangular model to a random group in the density model, at the same density. This goes as follows: Let $m$ be the number of generators used in the density model, let $\ell$ be a length which is a multiple of 3 , and let $W_{m, \ell / 3}^{\prime}$ be the set of all reduced words of length $\ell / 3$ in the symbols $a_{1}^{ \pm 1}, \ldots, a_{m}^{ \pm 1}$. Now take $n=\frac{1}{2} \# W_{m, \ell / 3}^{\prime}$ and define a map $\varphi$ from the free group $\left\langle b_{1}, \ldots, b_{n}\right\rangle$ to the free group $\left\langle a_{1}, \ldots, a_{m}\right\rangle$ by enumerating all the words in $W_{m, \ell / 3}^{\prime}$ and sending each $b_{i}$ to a distinct such word (and sending inverses to inverse words). Note that if $w \in W_{n, 3}$ then $\varphi(w)$ is a word of length $\ell$ in the $a_{i}^{ \pm 1}$ 's.

Now if $G_{3}=\left\langle b_{1}, \ldots, b_{n} \mid R_{3}\right\rangle$ is a random group in the triangular model, we can define a group $G=\left\langle a_{1}, \ldots, a_{m} \mid \varphi\left(R_{3}\right)\right\rangle$, in which the relators will have length $\ell$. If $G_{3}$ is taken at density $d$, then by definition it consists of $\left(\# W_{n, 3}\right)^{d} \sim(2 n)^{3 d}$ relators, so that $\# \varphi\left(R_{3}\right)=\# R_{3} \sim(2 n)^{3 d}=$ $\left(\# W_{m, \ell / 3}^{\prime}\right)^{3 d} \sim\left((2 m-1)^{\ell / 3}\right)^{3 d}=(2 m-1)^{3 d \ell}$, in accordance with the density model. Note also that the image of the uniform law on $W_{n, 3}$ is (almost) the uniform law on the set of reduced words of length $\ell$ in the $a_{i}^{ \pm 1}$ 's. (The "almost" comes from the fact that if $w=b_{i_{1}} b_{i_{2}} b_{i_{3}}$, then $\varphi(w)$ may not be reduced at the junction points of $\varphi\left(b_{i_{j}}\right)$ with $\varphi\left(b_{i_{j+1}}\right)$, but the density model is robust to such a slight modification, see $\S$ I.2.c.).

So, up to this latter technicality, there is a natural homomorphism $\varphi$ : $G_{3} \rightarrow G$ from a random group in the triangular model to a random group in the density model, at the same density. This means that the triangular model is "less quotiented" than the density one.

It is possible to prove [Żuk03] quite the same hyperbolicity theorem as for the density model:

THEOREM 29 - If $d<1 / 2$, then with overwhelming probability a random group in the triangular model, at density $d$, is non-elementary hyperbolic. If $d>1 / 2$, it is trivial with overwhelming probability. 
But the fact that groups in the triangular model are "larger" than those in the density model is especially clear when considering the following proposition.

Proposition 30 - If $d<1 / 3$, then with overwhelming probability, a random group in the triangular model at density $d$ is free.

Of course its rank is smaller than $n$. This results from the fact that at density $d<1 / 3$ in the triangular model, the dual graph of any van Kampen diagram is a tree.

Żuk [Żuk03] wrote a proof that in the triangular model, the spectral criterion for property $(T)$ is satisfied:

Theorem 31 - If $d>1 / 3$, then with overwhelming probability, a random group in the triangular model at density $d$ has property $(T)$.

In the triangular model, density $1 / 3$ corresponds to the number of relators being equal to the number of generators. So typically at $d>1 / 3$ every generator appears a large number of times in the relators, which is not the case for $d<1 / 3$. Consequently, the link of $e$ in the Cayley graph will be a random graph with a large number of edges per vertex. Such graphs have a very large (close to 1) spectral gap and the spectral criterion for property $(T)$ mentioned above applies.

Actually, as an intermediate step Żuk uses yet another variant of the triangular model (based on random permutations, see section 7.1 in [Żuk03]), which is rather artificial for random groups but arises very naturally in the context of random graphs, a crucial tool of the proof. The transfer to the standard triangular model involves use of the matching theorem.

Now property $(T)$ is stable under quotients. Using the morphism $\varphi$ : $G_{3} \rightarrow G$ above, Theorem 27 follows from Theorem 31 and from all the details we've omitted (e.g. the necessary modifications to make in order to get a surjective $\varphi$, or handling of the reduction problems). It seems that actually neither these details nor Theorem 27 itself do appear in the literature.

I.3.h. Testing the triangular model: Gromov vs. the computer. There is an amusing story to be told about the triangular model. In 2001, Richard Kenyon performed computer experiments to test Gromov's statement (Theorem 29). He used Derek Holt's KBMAG package [Hol95] to test triviality of random groups in the triangular model. The tests were made up to $n=500$ generators using about 2000 relations (which makes $d$ slightly above $1 / 3$ ). The results suggested that triviality occurred as soon as $d>1 / 3$, in contradiction with Theorem 29. Kenyon subsequently reviewed Gromov's proof of Theorem 11 given in [Gro93] and pointed out the omission of van Kampen diagrams featuring the same relator several times (see $\S$ V.). 
The author performed another series of experiments and analyzed the results by hand. It turned out that each time the group was trivial at $1 / 3<d<1 / 2$, this was due to some "exceptional event" whose asymptotic probability should be very small; but the combinatorial factor counting these events, although bounded, is quite large (a fact that may be related to the huge constants appearing in the local-global principle, see discussion in $\S \mathrm{V}$.); so the phenomenon should disappear when using larger $n$. On the other hand, it was not difficult to correct Gromov's argument (this led to the proof of Theorem 11 given in [Oll04]). The observed change of behavior of the algorithm at $d>1 / 3$ might be related to Proposition 30: at $d<1 / 3$ the group is non-trivial (actually free) for trivial reasons, whereas at $1 / 3<d<1 / 2$ the reasons for non-triviality involve the full strength of hyperbolic theory.

The triangular model has seemingly been less successful than the density model. Comparing Proposition 30 and Theorem 31, this model may be quite specific to the study of property $(T)$ (but a one-step proof of Theorem 27 using the spectral criterion applied to a generating set made of words of length $\ell / 3$ is feasible). Moreover the triangular model does not generalize to a theory of random quotients of given groups (§ II.1., § II.2.). On the contrary, in the usual model density controls the occurrence of several combinatorial and geometric events; we now turn to the description of some transformations happening at densities $1 / 6$ and $1 / 5$.

I.3.i. Cubical CAT(0)-ness and the Haagerup property. In view of Theorem 27 , one can wonder whether $1 / 3$ is the optimal density value for the occurrence of property $(T)$. It is not true that all random groups have this property: indeed, random groups at $d<1 / 12$ are $C^{\prime}(1 / 6)$ small cancellation groups, and, following Wise [Wis04], those do not have property $(T)$. This happens to be the case up to density at least $1 / 5$ [OW-b]:

Theorem 32 - Let $d<1 / 5$. Then with overwhelming probability, a random group at density $d$ has a codimension-1 subgroup. In particular, it does not have property $(T)$.

The codimension-1 subgroup (the existence of which excludes property $(T)$ by a result in [NR98]) is constructed through a technique developed by Sageev [Sag95], extended among others by Wise [Wis04], related to actions of the group on cube complexes. When $d<1 / 6$, the construction of [Wis04] fully applies and provides a complete geometrization theorem [OW-b]:

Theorem 33 - Let $d<1 / 6$. Then with overwhelming probability, a random group at density $d$ acts freely and cocompactly on a $C A T(0)$ cube complex. Moreover it is a-T-menable (Haagerup property).

Like property $(T)$, with which it is incompatible, the Haagerup property of a group has to do with its actions on the Hilbert space. It amounts to 
the existence of a proper isometric affine action on the Hilbert space, which is a kind of flexibility excluding property $(T)$. We refer to [CCJJV01] for a fact sheet on the Haagerup property. For discrete groups, a very nice equivalent definition is the existence of a proper action on a space with measured walls [CMV04].

The strategy is to construct walls [HP98] in the group. Natural candidates to be walls are hypergraphs [Wis04], which are graphs built from the Cayley complex as follows: the vertices of the hypergraphs are midpoints of edges of the Cayley complex, and the edges of the hypergraphs connect vertices corresponding to midpoints of diametrally opposite edges in faces of the Cayley complex (assuming that all relators have even length).

Densities $1 / 5$ and $1 / 6$ come into play as follows. If the group presentation satisfies the conclusion of Theorem 15 (kind of Greendlinger's Lemma), which happens up to $1 / 5$, then the hypergraphs are trees embedded in the Cayley complex and so are genuine walls. The stabilizers of these walls provide codimension-1 subgroups, thus refuting property $(T)$ [NR98]. On the contrary it happens that for $d>1 / 5$, there is only one hypergraph, which passes through every edge of the Cayley complex [OW-b]...

Following Sageev's [Sag95] original ideas, there is a now standard [NR98, Nic04, Wis04, CN, HW] correspondence between spaces with walls and cubical complexes. In our case, below density $1 / 6$ (but not above) the hypergraphs have some convexity properties and moreover two given hypergraphs cannot intersect at more than one point (except for degenerate cases); this allows to show that there are "enough" walls for the cube complex construction to work [HW], getting a free, cocompact action of the random group. (It seems likely however that Theorem 33 holds up to density $1 / 5$.)

The Haagerup property follows either from consideration of the cube complex as in [NR98], or from general properties of groups acting on spaces with walls ([CMV04], after a remark of Haglund, Paulin and Valette).

According to Proposition 10, random groups at $d<1 / 6$ satisfy the $C(6)$ small cancellation condition. So an interpretation of Theorem 33 is that "generic" $C(6)$ groups have the Haagerup property. It is currently an open question to know whether some $C(6)$ groups can have property $(T)$.

This closes our journey through the influence of density on properties of the group. Space was missing to draw the geometric pictures corresponding to the events considered; but each time, density allows or forbids the existence of very concrete diagrams in the Cayley complex with certain metric properties relevant to the question under study.

\section{I.4. The space of marked groups}

A group marked with $m$ elements is a finitely generated group $G$, together with an $m$-tuple of elements $g_{1}, \ldots, g_{m} \in G$ such that these elements gener- 
ate $G$; or, equivalently, a group $G$ together with a surjective homomorphism $F_{m} \rightarrow G$.

For fixed $m$, the space $\mathcal{G}_{m}$ of all groups marked with $m$ elements has a natural topology, apparently first introduced in Grigorchuk's celebrated paper [Gri84], part 6 (also compare the end of [Gro81]): two marked groups $\left(G,\left(g_{i}\right)\right)$ and $\left(G^{\prime},\left(g_{i}^{\prime}\right)\right)$ are close if the kernels of the two maps $F_{m} \rightarrow G$, $F_{m} \rightarrow G^{\prime}$ coincide in a large ball of $F_{m}$, or, equivalently, if some large balls in the Cayley graphs of $G$ and $G^{\prime}$ w.r.t. the two given generating sets are identified by the mapping $g_{i} \mapsto g_{i}^{\prime}$. This means that the two generating $m$-tuples have the same algebraic relations up to some large size.

We refer to [Pau03, Ch00, Gri84] for basic properties of the space $\mathcal{G}_{m}$. It is compact, totally discontinuous. Every finite group is an isolated point. The subspaces of Abelian groups, of torsion-free groups are closed. Finitely presented groups are dense in $\mathcal{G}_{m}$. Any finitely presented group has a neighborhood consisting only of quotients of it. The Minkowski dimension of $\mathcal{G}_{m}$ is infinite [Guy].

Isomorphism of groups defines a natural equivalence relation on $\mathcal{G}_{m}$. It happens that this relation is extremely irregular from a measurable point of view, so that it is not possible to measurably classify finitely generated groups by a real number [Ch91, Ch00]:

Theorem 34 - Let $m \geqslant 2$. There exists no Borel map $\mathcal{G}_{m} \rightarrow \mathbb{R}$ constant on the isomorphism classes and separating these classes.

Actually this equivalence relation is as irregular as a countable equivalence relation can be [TV99]. Let $X$ and $X^{\prime}$ be Borel sets and let $R, R^{\prime}$ be Borel equivalence relations on $X$ and $X^{\prime}$ respectively, with countable classes. Say that $R$ is reducible to $R^{\prime}$ if there exists a Borel map $f: X \rightarrow X^{\prime}$ such that $x R y \Leftrightarrow f(x) R^{\prime} f(y)$. In other words, $R^{\prime}$ is more complex than $R$. The theorem reads [TV99]:

Theorem 35 - Any Borel equivalence relation with countable classes is reducible to the isomorphism relation on $\mathcal{G}_{5}$.

A review of the results and uses of this space is beyond the scope of our work. In fact, it happens that the closure of the isomorphism class of the free group (the limit groups of Sela [Sel]) is already quite complex [Sel, CG05]. We focus here on the aspects linked to the idea of typicality for groups.

The usual notion of topological genericity $\left(G_{\delta}\right.$-dense sets $\grave{a} l a$ Baire) is not very interesting due to the totally discontinuous nature of the space; e.g. the set of Abelian groups is open-closed, as is any finite marked group alone, so that any Baire-generic property has to hold for these classes of groups. For these reasons, so far this space has not be used to define an alternate notion of a "typical" group competing with Gromov's probabilistic approach. 
Nevertheless, following Champetier we can use our prior knowledge of genericity of torsion-free hyperbolic groups (Theorems 5 and 11) to restrict ourselves to the closure in $\mathcal{G}_{m}$ of those groups, and try to identify generic properties therein. Indeed this program happens to work very well [Ch91, Ch00]:

Theorem 36 - Let $\mathcal{H}_{m}^{\text {tf }}$ be the closure in $\mathcal{G}_{m}$ of the subspace of all nonelementary, torsion-free hyperbolic groups. Then there is a $G_{\delta}$-dense subset $X \subset \mathcal{H}_{m}^{\text {tf }}$ such that any group $G \in X$ satisfies the following properties:

- Its isomorphism class is dense in $\mathcal{H}_{m}^{t f}$,

- It is torsion-free,

- It is of rank 2,

- It is of exponential growth, non-amenable,

- It contains no free subgroup of rank 2,

- It satisfies Kazhdan's property $(T)$,

- It is perfect,

- It has no finite quotient but the trivial group.

So all these properties can be viewed as generic properties of an "infinitely presented typical group".

Note however that such properties do depend on the class of groups we take the closure of. If, of Theorems 5 and 11, we had only retained the fact that a random group is non-elementary hyperbolic (and forget it is torsion-free), then we would naturally have considered the closure $\mathcal{H}_{m}$ in $\mathcal{G}_{m}$ of the subspace of non-elementary hyperbolic groups, in which case we get the following [Ch91, Ch00] (compare [Ols91c]):

Theorem 37 - There is a $G_{\delta}$-dense subset of $\mathcal{H}_{m}$ consisting only of groups which are infinite and all elements of which are of torsion.

Infinite torsion groups have long been sought for (Burnside problem dating back to 1902). They were constructed for the first time in 1964 by Adyan and Novikov and have been the source of an abundant literature since then (see e.g. [Iva98, Gup89] for reviews). Diagrammatic methods for this problem were introduced by Ol'shanskiu [Ols82, Ols83]. It seems that hyperbolic groups are a natural way towards infinite torsion groups ([DG], [IO96], [Iva94], [Ols93], [Ols91c], chapter 12 of [GhH90], section 4.5.C of [Gro87]).

The strength of this topological approach compared to the probabilistic one is that it gives access to infinitely presented groups. The drawback is that it does not provide by itself a non-trivial notion of generic properties of groups: one has to combine it with prior knowledge from the probabilistic approach. Once properties known to hold with overwhelming probability for finitely presented groups are selected (and the result may depend on this choice), the closure of these groups in the space $\mathcal{G}_{m}$ provides a notion of 
genericity for infinitely presented groups. Of course the notions of genericity for finitely and infinitely presented groups cannot but be mutually incompatible.

Note however that the probabilistic approach does not provide a welldefined notion of "random group" either since one has to consider a family of probability measures indexed by the length $\ell$ of the relators; but at least this defines a notion of a generic property of finitely presented groups when $\ell \rightarrow \infty$.

The temperature model (§ IV.k.), if understood, would solve all these problems, noticeably by providing a natural family of (quasi-invariant?) probability measures on $\mathcal{G}_{m}$. See also $\S$ IV.g. for questions arising in this framework. 


\section{Typical elements in a group}

Quoting from [Gro87], 5.5F: "Everything boils down to showing that adding 'sufficiently random' relations to a non-elementary word hyperbolic group gives us a word hyperbolic group again[...]" Considering random elements in a given group is often a good way to embody the intuition of which properties are true when "nothing particular happens" and when the elements are "unrelated". The behavior of random elements is often the best possible.

We illustrate this on two categories of examples: The first is that typical elements in a (torsion-free) hyperbolic group can be killed without harming the group too much (robustness of hyperbolicity), and the probabilistic approach allows to quantify very precisely the number of elements that can be killed. The second is a sharp counting of the number of one-relator groups up to isomorphism, the idea being that a random relator is nicely behaved, implying a rigidity property, and that by definition typical relators are the most numerous so that it is enough to count only them.

For this review, typical elements in a group were considered only insofar as they are put in some group presentation and provide new, randomlydefined groups, hence the two topics selected. Random elements in a finite or infinite group have plenty of interesting properties by themselves, which are not covered here. See for example the nice [Dix02] for the case of finite groups.

\section{II.1. Killing random elements of a group}

II.1.a. Random quotients by elements in a ball. Theorem 11 states that a random quotient of the free group is hyperbolic. One can wonder whether a random quotient of an already hyperbolic group stays hyperbolic, and this is the case. In other words, hyperbolicity is not only generic but also robust. This is all the more reasonable as, from a geometric point of view, the intuition is that (torsion-free) hyperbolic groups supposedly behave like free groups.

The following is Theorem 3 of [Oll04] (up to the benign replacement of spheres by balls, see $\S$ I.2.c.), which generalizes the phase transition of Theorem 11 above. As usual "with overwhelming probability" means "with probability tending to 1 as $\ell \rightarrow \infty$ ". 
Theorem 38 - Let $G_{0}$ be a non-elementary, torsion-free hyperbolic group equipped with some finite generating set, and let $B_{\ell}$ be the set of elements of $G_{0}$ with norm at most $\ell$ w.r.t. this generating set.

Let $0 \leqslant d \leqslant 1$. Let $R \subset G_{0}$ be a set obtained by picking at random $\left(\# B_{\ell}\right)^{d}$ times an element in $B_{\ell}$. Let $G=G_{0} /\langle R\rangle$ be the random quotient obtained.

- If $d<1 / 2$, then with overwhelming probability $G$ is (non-elementary) hyperbolic.

- If $d>1 / 2$, then with overwhelming probability $G=\{e\}$.

The explanation of the $1 / 2$ is of course exactly the same as for Theorem 11, namely the probabilistic pigeon-hole principle, although the proof for $d<1 / 2$ is considerably more difficult. Note again that the number $\left(\# B_{\ell}\right)^{d}$ is rather large. This phenomenon seems to be quite robust and general and might be generalized to other subsets in which the generators are picked, and maybe other classes of groups (§ IV.f.).

The torsion-freeness assumption can be relaxed to a "harmless torsion" one, but it cannot be completely removed, the obstruction being growth of the centralizers of torsion elements (see Theorem 41 below, $\S$ IV.f. and [Oll05b]).

We refer to $\S$ IV.f. for natural questions and open problems concerning these random quotients.

From a constructive point of view, it might seem quite difficult to pick random elements uniformly distributed in the ball of a group, compared to the easy generation of random words as used in Theorems 11 and 40 . However, algorithmic properties of hyperbolic groups are very nice: equality of two elements is decidable in real time [Hol00], every element can be efficiently $[\mathrm{EH}]$ put into a normal form, and there is an explicit finite automaton enumerating these normal forms ("Markov codings": section 5.2 of [Gro87], [GhH90]).

According to Gromov's quote above, the idea that unrelated elements in a hyperbolic group can be killed is quite old. In a deterministic context, this "relative small cancellation", presented in section 5.5 of [Gro87] (where Gromov refers to Ol'shanskiǔ's paper [Ols83]) was later formalized by Ol'shanskir (section 4 of [Ols93]), Champetier [Ch94] and Delzant [Del96a]. This theory generalizes the usual small cancellation $C^{\prime}(\lambda)$ to elements chosen in a hyperbolic group. But, just as usual small cancellation stops at density $1 / 12$ for random groups, relative small cancellation is too restrictive and does not make it up to the maximal number of elements one can kill, hence the interest of the random point of view.

II.1.b. Growth of random quotients. A theorem stating that the growth exponent does not change much under such a quotient, generalizing Theorem 25, has been proven [Oll-b] (we refer to $§$ I.3.e. for the definition 
of the growth exponent). Note that by the results in [AL02], this exponent cannot stay unchanged.

Theorem 39 - Let $G_{0}$ be a non-elementary, torsion-free hyperbolic group generated by the finite set $S$. Let $B_{\ell}$ be the ball in $G_{0}$ and $g$ the growth exponent of $G_{0}$, both w.r.t. $S$. Let $G$ be a random quotient of $G_{0}$ by elements of $B_{\ell}$ at density $d$ as in Theorem 38, and suppose of course $d<1 / 2$.

Then, for any $\varepsilon>0$, with overwhelming probability the growth exponent of $G$ lies in the interval $(g-\varepsilon ; g)$.

It is likely [Oll-e] that the spectral radius of the random walk operator on the group does not change much too (compare Theorem 42).

\section{II.2. Killing random words, and iterated quo- tients}

II.2.a. Random quotients by words. Theorem 38 describes what happens when quotienting a hyperbolic group by random elements in it. Another possible generalization of Theorem 11 is to quotient by random words in the generators. Though maybe not as intrinsic, this model has the advantage that the notion of random quotient becomes independent of the initial group (within the class of marked groups); in particular, it allows to study successive random quotients of a group taken w.r.t. one and the same generating set, as used notably in [Gro03].

Of course, the unavoidable consequence of the model being independent on the initial group is that the critical density will depend on this group. Actually the critical density is equal to the exponent of return to $e$ of the simple random walk w.r.t. the generating set $a_{1}, \ldots, a_{m}$ considered: basically, if this probability behaves like $(2 m)^{-\alpha t}$ for large times $t$, the critical density will be $\alpha$. The result reads ([Oll04], Theorem 4):

Theorem 40 - Let $G_{0}$ be a torsion-free hyperbolic group generated by the elements $a_{1}, \ldots, a_{m}$.

Let $\left(w_{t}\right)_{t \in \mathbb{N}}$ be the trajectory of a simple random walk in $G_{0}$ w.r.t. the generators $a_{i}^{ \pm 1}$ and let

$$
d_{\text {crit }}=-\lim _{\substack{t \rightarrow \infty \\ t \text { even }}} \frac{1}{t} \log _{2 m} \operatorname{Pr}\left(w_{t}=G_{0} e\right)=-\log _{2 m} \rho\left(G_{0}\right)
$$

where $\rho$ is the spectral radius of the random walk operator ([Kes59] or $\S$ I.3.f.). Note that $d_{\text {crit }}>0$ unless $G_{0}$ is elementary.

Let $0 \leqslant d \leqslant 1$ and let $W_{\ell}$ be the set of all $(2 m)^{\ell}$ words of length $\ell$ in the $a_{i}^{ \pm 1}$ 's. Let $R$ be the random set obtained by picking $(2 m)^{d \ell}$ times at random a word in $W_{\ell}$. 
Let $G=G_{0} /\langle R\rangle$ be the random quotient so obtained. Then with overwhelming probability:

- If $d<d_{\text {crit }}$, then $G$ is (non-elementary) hyperbolic.

- If $d>d_{\text {crit }}$, then $G=\{e\}$.

Once again the spirit of the density model is to kill a number of words equal to some power $d$ of the total number of words $(2 m)^{\ell}$. Note that $d_{\text {crit }}<1 / 2$, even when $G_{0}$ is the free group (the difference with Theorem 11 being that we use plain words instead of reduced ones).

There is also a version of Theorem 40 using reduced words instead of plain words (thus a formal generalization of Theorem 11). In this version (Theorem 2 of [Oll04]), $2 m$ is to be replaced with $2 m-1$ everywhere and a $\mathbb{Z} / 2 \mathbb{Z}$ might appear for $d>d_{\text {crit }}$ and even $\ell$. The critical density is now equal to $1 / 2$ for the free group, and to the exponent of return to $e$ of the reduced random walk in $G_{0}$ (i.e. the random walk with no immediate backtracks) for a non-free hyperbolic group $G_{0}$, which is equal to 1 minus the cogrowth exponent of $G_{0}$ [Oll04].

Theorems 38 and 40 are of course not proven independently. Section 4 of [Oll04] extracts axioms under which quotients of a hyperbolic group by elements taken under some probability measure yield a hyperbolic group again. These axioms have to do with exponents of large deviations of the measure.

Lots of open problems concerning random quotients of hyperbolic groups are stated in $\S$ IV.f.

II.2.b. Harmful torsion. As briefly mentioned above, the torsionfreeness assumption can be relaxed to a "harmless torsion" one demanding that the centralizers of torsion elements are either finite, or virtually $\mathbb{Z}$, or the whole group [Oll04]. But in [Oll05b] we give an example of a hyperbolic group with "harmful" torsion, for which Theorem 40 does not hold; moreover its random quotients actually exhibit three genuinely different phases instead of the usual two.

Theorem $41-$ Let $G_{0}=\left(F_{4} \times \mathbb{Z} / 2 \mathbb{Z}\right) \star F_{4}$ equipped with its natural generating set, where $\star$ denotes a free product. Let $d_{\text {crit }}$ be defined as above. Then there exists a density $0<d_{\text {crit }}^{\prime}<d_{\text {crit }}$ such that quotients of $G_{0}$ by random words at density $d>d_{\text {crit }}^{\prime}$ are trivial with overwhelming probability.

What happens is that above some density corresponding to the probability with which the random walk in $G_{0}$ sees the factor $F_{4} \times \mathbb{Z} / 2 \mathbb{Z}$, the factor $\mathbb{Z} / 2 \mathbb{Z}$ becomes central in the random quotient, so that above this density random quotients of $\left(F_{4} \times \mathbb{Z} / 2 \mathbb{Z}\right) \star F_{4}$ actually behave like random quotients of $F_{8} \times \mathbb{Z} / 2 \mathbb{Z}$, which has a lower critical density. 
A more careful analysis reveals the presence of two genuinely different phases for random quotients of $\left(F_{4} \times \mathbb{Z} / 2 \mathbb{Z}\right) \star F_{4}$ in addition to the trivial phase, depending on whether or not the $\mathbb{Z} / 2 \mathbb{Z}$ factor is central in the quotient. Elaborating on this construction, hyperbolic groups with torsion whose random quotients exhibit more than three different phases can probably be built. We refer to [01105b] for the details.

II.2.c. Cogrowth of random quotients, and iterated quotients. Since the critical density of the initial group $G_{0}$ is controlled by the spectral radius of the random walk operator, one might wonder what is the new value of this spectral radius for the random quotient (in particular, if it stays small enough, then we can take a new random quotient at a larger length). The answer from [Oll05a] (using results of [Ch93]), generalizing Theorem 26, is that it stays almost unchanged:

Theorem 42 - Let $G_{0}$ be a torsion-free hyperbolic group generated by the elements $a_{1}, \ldots, a_{m}$. Let $\rho\left(G_{0}\right)$ be the spectral radius of the random walk operator on $G_{0}$ w.r.t. this generating set; let $d_{\text {crit }}=-\log _{2 m} \rho\left(G_{0}\right)$ and let $G$ be a quotient of $G_{0}$ by random words at density $d<d_{\text {crit }}$ as in Theorem 40.

Then, for any $\varepsilon>0$, with overwhelming probability the spectral radius $\rho(G)$ of the random walk operator on $G$ w.r.t. $a_{1}, \ldots, a_{m}$ lies in the interval $\left(\rho\left(G_{0}\right) ; \rho\left(G_{0}\right)+\varepsilon\right)$.

The same theorem holds for quotients by random reduced words, and, very likely [Oll-e], for quotients by random elements of the ball as in Theorem 38 .

As a corollary, we get that the critical density for the new group $G$ is arbitrarily close to that for $G_{0}$. So we could take a new random quotient of $G$, at least if we knew that $G$ is torsion-free. This is not known ( $§$ IV.f.), but the results of [Oll04] imply that if $G_{0}$ is of geometric dimension 2 then so is $G$. So in particular, taking a free group for $G_{0}$ and iterating Theorem 40 we get:

Proposition 43 - Let $F_{m}$ be the free group on $m$ generators $a_{1}, \ldots, a_{m}$. Let $\left(\ell_{i}\right)_{i \in \mathbb{N}}$ be a sequence of integers. Let $d<-\log _{2 m} \rho\left(F_{m}\right)$ and, for each $i$, let $R_{i}$ be a set of random words of length $\ell_{i}$ at density $d$ as in Theorem 40.

Let $R=\bigcup R_{i}$ and let $G=F_{m} /\langle R\rangle$ be the (infinitely presented) random group so obtained.

Then, if the $\ell_{i}$ 's grow fast enough, with probability arbitrarily close to 1 the group $G$ is a direct limit of non-elementary hyperbolic groups, and in particular it is infinite.

It is not easy to follow the details of [Oll04, Oll05a, Ch93] closely enough to obtain an explicit necessary rate of growth for the $\ell_{i}$ 's, although $\ell_{i+1} \geqslant$ Cst. $\ell_{i}$ is likely to work. 
The techniques used in [Gro03] to get iterated quotients are different from those of [Oll05a] and of more geometric inspiration (see $\S$ III.2. or [Gro01a, Oll-c]); in particular, therein property $(T)$ is used to gain uniform control on the critical densities of all successive quotients. The drawback is that these techniques only work for very small densities.

\section{II.3. Counting one-relator groups}

On a very different topic, consideration of generic-case rather than worstcase behavior for algorithmic problems in group theory (most notably the isomorphism problem) led I. Kapovich, Myasnikov, Schupp and Shpilrain, in a series of closely related papers [KSS, KS, KS05, KMSS05, KMSS03], to the conclusion that generic elements are often nicely behaved. The frontier between properties of one-relator groups and properties of a typical word in the free group is faint; for this review we selected an application where the emphasis is really put on the group, namely, evaluation of the number of distinct one-relator groups up to isomorphism.

The isomorphism problem for finite presentations is generally undecidable (see e.g. the very nice [Sti82] for an introduction to the word and isomorphism problem, or the end of chapter 12 of [Rot95]). It has been solved for the class of torsion-free hyperbolic groups with finite outer automorphism group ([Sel95], see also [Pau91]), which contains generic one-relator groups since their outer automorphism group is trivial [KSS]. However, having an algorithm for the isomorphism problem does not provide an estimate of the number of isomorphism classes.

For one-relator groups, the basic idea is as follows: Since generic relators are by definition much more numerous than particular relators, if we can show that one-relator groups with a generic enough relator are mutually non-isomorphic, then we will get a sharp estimate of the number of isomorphism classes of one-relator groups.

Let $I_{\ell}(m)$ be the number of isomorphism classes of one-relator group presentations $\left\langle a_{1}, \ldots, a_{m} \mid r\right\rangle$ with $|r| \leqslant \ell$. Of course $I_{\ell}(m)$ is less than the number of cyclically reduced words of length at most $\ell$; this crude estimate can be improved since taking a cyclic permutation of $r$ does not change the group. Now the number of cyclically reduced words of length $\leqslant \ell$ up to cyclic permutation is about $(2 m-1)^{\ell} / \ell$. Moreover, some trivial symmetries (such as exchanging the generators $a_{1}, \ldots, a_{m}$ or taking inverses) decrease this estimate by some explicit factor depending only on $m$. Actually the estimate found this way is sharp [KS, KSS]:

Theorem 44 - The number $I_{\ell}(m)$ of isomorphism classes of one-relator groups on $m$ generators, with the relator of length at most $\ell$, satisfies

$$
I_{\ell}(m) \sim \frac{1}{m ! 2^{m+1}} \frac{(2 m-1)^{\ell}}{\ell}
$$


when $\ell \rightarrow \infty$.

Once Theorem 19 is known the result is relatively simple. Indeed, a theorem of Magnus ([LS77], Prop. II.5.8) implies that if two elements of the free group generate the same normal subgroups then they are the same up to conjugation and inversion. If two generic one-relator groups are isomorphic, then Theorem 19 implies that after applying some automorphism of the free group, the two relators have the same normal closure, and thus are essentially the same by Magnus' Theorem. So the isomorphism problem for generic one-relator groups reduces to the problem of knowing when an element in the free group is the image of another under some automorphism of the free group.

The orbits of the automorphism group of a free group are well studied and generated by so-called Whitehead moves ([LS77], I.4): especially, if two elements lie in the same orbit and are of minimal length within this orbit, they can be transformed into each other by means of non-length-increasing Whitehead moves. But for generic elements it can be easily shown that the action of the Whitehead moves increases length except for some trivial cases, so that generic elements do not lie in the same orbit.

In other words, a generic element cannot be "simplified" by action of automorphisms of the free group. This same minimal representation idea allowed to get an estimate [KS] of Delzant's $T$-invariant for generic onerelator group (this invariant, defined in [Del96b], attempts to measure the minimal possible "complexity" of presentations of a given group).

The only (but crucial) place above where one-relatorness plays a role is the use of the Magnus theorem, which has no known replacement for the case of several relators (even generic ones). On the other hand, genericity really lies at the core of the argument: the idea is that for counting matters, particular annoying cases can be discarded and only the nicest, typical cases can be treated. 


\section{Applications: Random ingredients in specific constructions}

What non-probabilists call "the probabilistic method" is the use of random constructions to prove existence theorems and to build new objects and (counter-)examples. Badly enough, this is often seen as the only possible justification for the introduction of random tools in a classical field.

Random groups fit into this scheme. Up to now, the main application of random groups is the construction by Gromov [Gro03] of a finitely presentable group whose Cayley graph (quasi-)contains an infinite family of expanding graphs and which contradicts the Baum-Connes conjecture with coefficients [HLS02]. We give the roadmap to this construction in $\S$ III.1. and $\S$ III.2.

A second application (§ III.3.), using the same tools together with a construction of Rips [Rip82], allowed in [OW-a] to construct Kazhdan groups whose outer automorphism group contains an arbitrary countable group, answering a question of Paulin (in the list of open problems in [HV89]). As was noted by Cornulier [Cor-b], this implies in particular that any discrete group with property $(T)$ is a quotient of a torsion-free hyperbolic group with property $(T)$. The technique is flexible and provides other examples of Kazhdan groups with prescribed properties.

Let us insist that groups constructed this way cannot pretend to "typicality": in each case the random constructions are twisted in ways specific to the goal to achieve. The process of building a group containing a family of expanders starts with the choice of such a family and uses it to define the group; the expanders do not appear out of the blue in a plain random group (compare the techniques in [AC04], though: it may be that Cayley graphs of plain random groups contain lots of interesting families of graphs).

The common tool to both constructions above is Gromov's powerful and flexible generalization of small cancellation theory to group presentations arising from labelled graphs. When everything goes well, the said graph embeds in the Cayley graph of the group, thus allowing "shaping" of Cayley graphs. Moreover, this extension of small cancellation is compatible with property $(T)$, whereas usual small cancellation is not [Wis04], showing that a really new class of groups is accessible this way. 


\section{III.1. Shaping Cayley graphs: graphical pre- sentations}

Before we state the results, let us describe this graphical presentation tool. It is discussed in the last paragraph, "Random presentations of groups", in [Gro00], and more thoroughly in sections 1 and 2 of [Gro03]. (The idea of representing subgroups of the free group by labelled graphs goes back to [Sta83], but therein the emphasis is on the subgroup and not on the quotient group "presented" by the graph.)

III.1.a. Labelled graphs and group presentations. Let us state some vocabulary geometrizing group presentations. Let $a_{1}, \ldots, a_{m}$ be our usual generators and let $B$ be the following standard labelled graph: $B$ consists of one single vertex and $m$ oriented loops, univocally labelled with the generators $a_{1}, \ldots a_{m}$. Now a word in the $a_{i}^{ \pm 1}$ is simply a path in the labelled graph $B$. A reduced word is an immersed path $P \leftrightarrow B$.

A labelled graph is a graph $\Gamma$ together with a graph map $\Gamma \rightarrow B$, i.e. a graph in which every edge bears a generator $a_{i}$ with an orientation. It is said to be reduced if this map is an immersion; this amounts to not having two distinct edges with identical labels originating (or ending) at the same vertex (this is called "folded" in [Sta83], but this terminology is less consistent with the case of reduced words, reduced van Kampen diagrams, etc.).

Gromov's idea is that to a labelled graph we can associate the group presentation whose relators are all the words read on cycles of the graph. More precisely, let $\Gamma$ be a labelled graph and let $x_{0}$ be any basepoint in $\Gamma$. The labelling $\varphi: \Gamma \rightarrow B$ defines a map $\varphi: \pi_{1}\left(\Gamma, x_{0}\right) \rightarrow F_{m}$, sending a closed path to its label. The group presented by $\Gamma$ is by definition the group

$$
G=\left\langle a_{1}, \ldots, a_{m} \mid \Gamma\right\rangle=F_{m} /\left\langle\varphi\left(\pi_{1}(\Gamma), x_{0}\right)\right\rangle
$$

(when $\Gamma$ is not connected, this is defined as $G=F_{m} /\left\langle\cup \varphi\left(\pi_{1}\left(\Gamma, x_{i}\right)\right)\right\rangle$ taking a basepoint in each connected component). If $\pi_{1}(\Gamma)$ is generated by the cycles $\left(c_{i}\right)_{i \in I}$ then a cheaper presentation for $G$ is

$$
G=\left\langle a_{1}, \ldots, a_{m} \mid \varphi\left(c_{i}\right)_{i \in I}\right\rangle
$$

and note that $I$ can be taken finite if $\Gamma$ is finite. Note also that changing the basepoint amounts to taking some conjugate of the image $\varphi\left(\pi_{1}(\Gamma)\right)$, so that the group defined by $\Gamma$ is unchanged. We will call $\left\langle a_{1}, \ldots, a_{m} \mid \Gamma\right\rangle$ a graphical presentation for $G$.

The group $G$ is of course the fundamental group of the 2-complex obtained by gluing a disk in $B$ along each of the paths $\varphi\left(c_{i}\right)$ where the $c_{i}$ are the simple cycles of $\Gamma$. 
Most importantly, when $\Gamma$ consists of a disjoint union of circles, then we get back the usual notion of group presentation. The relators are cyclically reduced if and only if the labelling is reduced.

The Cayley graph $\operatorname{Cay}\left(G,\left(a_{i}\right)\right)$ is itself a labelled graph. By definition of $G$, the label of any cycle in $\Gamma$ is a relation in $G$ and so can be read on some closed path in the Cayley graph. Consequently, if we fix a basepoint $x_{i}$ in each connected component of $\Gamma$, and any basepoint $y \in \operatorname{Cay}\left(G,\left(a_{i}\right)\right)$, then there is a unique label-preserving map

$$
\varphi: \Gamma \rightarrow \operatorname{Cay}\left(G,\left(a_{i}\right)\right)
$$

sending each $x_{i}$ to $y$, which we denote (again!) by $\varphi$ since it commutes with the labelling maps to $B$.

Of course nothing guarantees that in general this map will be injective. It could happen that $G$ is trivial, for example. But if $\varphi$ is injective, then we have succeeded in embedding a graph in the Cayley graph of some group. This is what Gromov did with $\Gamma$ a family of expanders, as we will describe in $\S$ III.2. For the moment, we turn to the description of a small cancellation condition ensuring this injectivity.

III.1.b. Graphical small cancellation. The central notion of small cancellation is that of piece: a piece is a word that can be read twice in the relators of a presentation. Here this notion generalizes as follows: Let $\Gamma \stackrel{\varphi}{\longrightarrow} B$ be a labelled graph. A piece in $\Gamma$ is a word $P \stackrel{\psi}{\rightarrow} B$ which can be read at two different places on $\Gamma$, that is, such that there are two distinct immersions $P \stackrel{i_{1}}{\rightarrow} \Gamma \stackrel{\varphi}{\rightarrow} B$ and $P \stackrel{i_{2}}{\rightarrow} \Gamma \stackrel{\varphi}{\rightarrow} B$ (preserving the labels, of course, i.e. $\varphi \circ i_{1}=\varphi \circ i_{2}=\psi$ ).

What matters for small cancellation is the size of pieces compared to the size of the relators on which they appear. Here the role of relators is played by cycles in the graph. So we define the relative length of a piece $P$ to be the maximum of the ratio $|P| /|C|$ over all immersed cycles $C \uparrow \Gamma$ such that $P$ appears on $C$ i.e. there exists $P \leftrightarrow C \leftrightarrow \Gamma$.

Definition 45 - A labelled graph $\Gamma$ satisfies the graphical small cancellation condition $G r^{\prime}(\alpha)$ if the relative length of any piece in $\Gamma$ is less than $\alpha$.

It should be clear that when $\Gamma$ is a disjoint union of circles, this reduces to the traditional $C^{\prime}(\alpha)$ small cancellation condition. The well-known $C^{\prime}(1 / 6)$ theory extends to the new framework. Similarly one could define the combinatorial $\operatorname{Gr}(p)$ condition asking for no cycle in $\Gamma$ to be the union of fewer than $p$ pieces.

The following is a much simplified version of the statements in section 2.2 of [Gro03] (see $\S$ III.1.e. below for a more general setting). Gromov uses general geometric arguments, but the version presented here is easy to prove 
using the usual combinatorial techniques of small cancellation (see [Oll-a] or [Wis]).

Theorem 46 - Let $\Gamma$ be a labelled graph which is spurless (i.e. with no valency-1 vertex) and reduced. Suppose that $\Gamma$ satisfies the $G r^{\prime}(1 / 6)$ graphical small cancellation condition.

Then the group $G$ defined by the graphical presentation $\Gamma$ enjoys the following properties:

- If $\Gamma$ is finite, $G$ is hyperbolic; if $\Gamma$ is infinite $G$ is a direct limit of hyperbolic groups.

- It is torsion-free, of geometric dimension 2, of Euler characteristic $1-m+b_{1}(\Gamma)$. In particular if $b_{1}(\Gamma)>m$ it is infinite and not free.

- The natural map of labelled graphs from $\Gamma$ to the Cayley graph of $G$ (for any basepoint choice) is an isometric embedding for the graph distances; in particular it is injective.

- The length of the shortest cycle in the Cayley graph is equal to that in $\Gamma$.

More properties of usual small cancellation still hold, such as asphericity of the "standard" presentation and a kind of Greendlinger lemma (see details in [Oll-a]).

The reducedness assumption is necessary: otherwise we could add arbitrary long paths labelled by words which are trivial in the free group, thus artificially decreasing the relative length of pieces by increasing the length of cycles. Spurs do not change the group defined by the graph, but might not embed isometrically, unless $G r^{\prime}(1 / 8)$ holds.

The idea of one of the possible proofs is to consider van Kampen diagrams all faces of which bear a word read on some cycle of the graph, and are "minimal" in the sense that the word read on the boundary of the union of two adjacent faces is not read on a cycle of the graph (otherwise we merge the two faces). Such "minimal" diagrams (locally) satisfy the usual $C^{\prime}(1 / 6)$ condition hence a linear isoperimetric inequality (w.r.t. to the set of relators made of all words read on cycles of the graph), and hyperbolicity follows by the remark that any cycle in the graph can be written as a concatenation of linearly many cycles of bounded sizes, so that we can replace this infinite presentation by a finite one while keeping a linear isoperimetric inequality. Asphericity, cohomological dimension and isometric embedding of the graph into the Cayley graph require a little more work. Although this basic idea is simple, there are some delicate topological details [Oll-a].

III.1.c. Random labellings are $G r^{\prime}(1 / 6)$. One of the interests of the $G r^{\prime}$ condition is that random labellings of a graph satisfy it very probably. A random labelling of a graph is simply the choice, for each edge of the graph, of a generator $a_{i}$ together with an orientation, picked at random 
among the $2 m$ such possible choices. Generally this does not result in a reduced labelling, but we can reduce the graph by performing the necessary edge identifications (the "folding" of [Sta83]).

Of course, if there are "too many" cycles in the graph, the group will tend to be trivial. According to the spirit of the density model (Def. 7), this "too many" has to be defined with respect to the length of the cycles: the longer the cycles, the more of them we can tolerate. A way to achieve this is to subdivide the graph, i.e. replace each edge with 100 (say) consecutive edges. For a given unlabelled graph $\Gamma$, we will denote $\Gamma^{/ j}$ its $j$-subdivision (each edge is replaced with $j$ edges). Another interpretation of this is to use edge labels which are length- $j$ words in the generators instead of single generators. Subdividing amounts to decreasing the "density" of the graph, which decreases the expected size of pieces (compare equation $(*)_{\tau}$ in section 1.2 of [Gro03] with a density). The same effect could be achieved by increasing the number $m$ of generators.

We give here an oversimplified version of statements in paragraphs 1.1, 4.6 and 4.8 of [Gro03] (which deal, much more generally, with random quotients of hyperbolic groups by graphical presentations, see $\S$ III.1.e.). A proof of this particular case can be found in [OW-a]. We will need some "bounded geometry" assumptions on the graph, bounding the valency of vertices and the diameter/girth ratio. The girth of a graph is defined as the length of the shortest non-trivial cycle in it. It plays the role of the length of the relators in the density case.

Proposition 47 - For any $\alpha>0$, for any number of generators $m \geqslant 2$, for any $v \in \mathbb{N}$ and $C \geqslant 1 / 2$ there exists an integer $j_{0}$ such that for any $j \geqslant j_{0}$ the following holds:

For any graph $\Gamma$ satisfying the following conditions:

- The valency of any vertex of $\Gamma$ is at most $v$.

- The girth and diameter of $\Gamma$ satisfy Diam $\Gamma \leqslant C$ girth $\Gamma<\infty$.

then a random labelling of the $j$-subdivision $\Gamma^{/ j}$, once reduced, satisfies the $G^{\prime}(\alpha)$ condition, with probability arbitrarily close to 1 if girth $\Gamma$ is large enough (depending on $\alpha, v, C$ ).

In particular for $\alpha<1 / 6$ the conclusions of Theorem 46 hold.

Moreover, the metric distortion induced by the reduction step is controlled (see the last section of [Oll-a], or [OW-a]).

III.1.d. Random labellings of expanders entail Kazhdan's property $(T)$. The group defined by a graphical presentation inherits some spectral properties of the graph, at least when the labelling is random. In particular, if the Laplacian on the graph has a large enough spectral gap, then the group defined by a random labelling will have property $(T)$. Section 1.2 of [Gro03] mentions (generalizations of) this, and the whole 
section 3 of [Gro03] is devoted to building a general framework encompassing the usual spectral criteria for property $(T)$ mentioned above in I.3.g. (see references there). We give only the following statement, a detailed proof of which was written by Silberman ([Sil03], Corollary 2.19):

Theorem 48 - Given $v \in \mathbb{N}, \lambda_{0}>0$ and an integer $j \geqslant 1$ there exists an explicit $g_{0}$ such that if $\Gamma$ is a graph with girth $\Gamma \geqslant g_{0}, \lambda_{1}(\Gamma) \geqslant \lambda_{0}$ and every vertex of which has valency between 3 and $v$, then a random labelling of the $j$-subdivision $\Gamma^{/ j}$ defines a group with Kazhdan's property $(T)$, with probability tending to 1 as the size of $\Gamma$ tends to infinity.

The idea is as follows: Property $(T)$ is related to the way the random walk operator acts on equivariant functions from the group to unitary representations of it. Now in the case of graphical presentations, by construction any equivariant function on the group (which is determined by its value at e) can be lifted to a "label-equivariant" function on the graph since cycles in the graph are labelled by relations in the group. If moreover the labelling was taken at random, then a random walk in the graph "simulates" a random walk in the group in the sense that the labels encountered by a random walk in the graph are plain random words (at short times). So if the graph has a large spectral gap, it is possible to transfer the spectral inequality to the random walk operator on the group. The details can be found in [Sil03].

Note that the first step (lifting equivariant functions) follows only from the definition of graphical presentations, whereas the second one uses the fact that the labelling was random (in some weak, statistically testable sense).

III.1.e. Generalizations: relative graphical presentations, and more. A labelled graph can also be used to define a quotient of an arbitrary marked group, by quotienting the group by the words read on cycles of the graph. This is a key step used by Gromov in the wild group construction described below (§ III.2.).

Just as ordinary small cancellation theory can be extended from quotients of the free group to quotients of a given hyperbolic group by elements satisfying a "relative small cancellation" condition ([Del96a], [Ch94], section 4 of [Ols93], section 5.5 of [Gro87]), an analogue to Theorem 46 holds when the initial group is hyperbolic (maybe with some restriction on torsion) instead of free.

In [Oll-c] an elementary version of Gromov's statements is given, which can be proven using the traditional van Kampen diagram approach of [Oll04], combined with the combinatorial arguments specific to the graphical case as in [Oll-a].

But Gromov proved this in a more general context using "rotation families of groups", where purely geometrical arguments can be given. The context is a group $G$ acting properly and cocompactly by isometries on 
some hyperbolic space $X$; we want to study the quotient of $G$ by a normal subgroup $R$.

In non-graphical small cancellation theory (relative to a hyperbolic group $G), R$ is generated by elements $\left(u_{i}\right)$ and all their conjugates; with each $u_{i}$ is associated a geodesic $U_{i}$ in $X$ invariant under $u_{i}$; a conjugate of $u_{i}$ will be associated with the corresponding translate of $U_{i}$. Small cancellation for the family $\left(u_{i}\right)$ (relative to $G$ ) is equivalent to the family of all $U_{i}$ 's and their translates not to travel close to each other for a "too long" time (the time is measured w.r.t. the minimal displacement of the action of the $u_{i}$ 's on $X$, namely, less than $1 / 6$ of this displacement; closeness is measured w.r.t. the hyperbolicity constant of $X$ ). For graphical small cancellation, say we have a connected labelled graph $\Gamma$; lift its universal cover $\tilde{\Gamma}$ to $G$ and take the corresponding orbit $U$ in $X$ (this is a tree), together with all its translates (the translates correspond to conjugate lifts of $\tilde{\Gamma}$ to $G$ ); if this family of trees in $X$ satisfies the same condition as above (not travelling close to each other for a long time), then the quotient of $G$ by the labelled graph will be hyperbolic again. If $\Gamma$ is not connected we get as many $U_{i}$ 's as there are connected components (plus their translates).

In section 2 of [Gro03], Gromov exposes a (difficult to read) general terminology and conditions for these ideas to work. Elements of proof are scattered in four papers (section 2 of [Gro03], sections 6-7 of [Gro01a], sections 25-32 of [Gro01b], section 10 of [Gro01c]). This framework seems to be quite powerful.

A simpler proof can be given in the case of very small cancellation (with $1 / 6$ replaced by some tiny constant), using $\mathrm{CAT}(-1, \varepsilon)$ spaces. The idea of the proof, very neatly described at the beginning of [DG] (see also [Del-b] and Gromov's papers just cited) and fully developed later in that paper, is as follows: we have a group $G=\pi_{1}(X)$ acting properly cocompactly by isometries on a hyperbolic space $\tilde{X}$, and we want to quotient $G$ by a normal subgroup $R$; the quotient is, of course, the fundamental group of the space $X^{\prime}$ obtained by gluing disks to $X$ along loops in $X$ corresponding to generators of the normal subgroup $R$. The idea is to endow these disks with a metric of constant negative curvature turning them into hyperbolic cones. This allows to check that $X^{\prime}$ is locally a CAT $(-1, \varepsilon)$ space, and the Cartan-Hadamard theorem (or local-global principle for hyperbolic spaces) then allows to conclude that the universal cover $\widetilde{X^{\prime}}$ is globally $\operatorname{CAT}(-1, \varepsilon)$, hence hyperbolicity of $G / R$.

This idea of metrizing Cayley complexes, applied in [DG] to the Burnside problem, looks very promising (see $§$ IV.i.).

\section{III.2. Cayley graphs with expanders}

In [Gro03] (as announced in [Gro00]), Gromov constructs a finitely generated group whose Cayley graph "quasi-contains" a family of expanding 
graphs and which thus admits no uniform embedding into the Hilbert space. The main idea is to use a graphical presentation arising from a random labelling of these expanders.

Recall (see e.g. [Lub94], [DSV03]) that a family of expanding graphs (or expanders) is a sequence of graphs $\left(\Gamma_{i}\right)_{i \in \mathbb{N}}$ of bounded valency, of size tending to infinity, such that the first eigenvalue of the discrete Laplacian on them is bounded away from 0 when $i \rightarrow \infty$. A uniform embedding of metric spaces is a map $\varphi$ such that there exists a function $f: \mathbb{R} \rightarrow \mathbb{R}$ with $\operatorname{dist}(\varphi(x), \varphi(y)) \geqslant f(\operatorname{dist}(x, y))$ and $f(x) \rightarrow \infty$ when $x \rightarrow \infty$.

One of the reasons for the interest in this paper is that, as proven by Higson, V. Lafforgue and Skandalis in [HLS02], this implies failure of the Baum-Connes conjecture with coefficients for this group. The initial stronger motivation was to refute the Novikov conjecture. Introducing these conjectures is beyond the scope of this paper and the author's field of competence. We refer the reader to [KL05, Val02b, Ska99, Hig98]. Gromov's group is a direct limit of hyperbolic groups; for hyperbolic groups, the Novikov conjecture [CM88, CM90], existence of a uniform embedding into the Hilbert space [Sel92] and the Baum-Connes conjecture [Laf02, MY02] hold. For the link between those last two properties see [Yu00, STY02].

Theorem 49 - For any $\varepsilon>0$ there exists a finitely generated, recursively presented group $G$, a family of expanders $\left(\Gamma_{i}\right)_{i \in \mathbb{N}}$, constants $A, B>0$ and maps $\varphi_{i}$ sending the vertices of $\Gamma_{i}$ to vertices of Cay $(G)$ such that

$$
A\left(\operatorname{dist}(x, y)-\varepsilon \operatorname{Diam} \Gamma_{i}\right) \leqslant \operatorname{dist}\left(\varphi_{i}(x), \varphi_{i}(y)\right) \leqslant B \operatorname{dist}(x, y)
$$

for any $i$ and $x, y \in \Gamma_{i}$, where the distance in $\Gamma_{i}$ is the ordinary graph distance and the distance in $\operatorname{Cay}(G)$ is w.r.t. some fixed finite generating set.

Consequently there exists a finitely presented group admitting no uniform embedding into the Hilbert space.

All the ingredients of the proof can be found in [Gro03], though lots of details are omitted. Gromov apologizes in the introduction that he chose not to write "a few technical lemmas, with a straightforward half-page proof each" but rather to "uncover the proper context rendering [...] the proofs tautological", and then adds "A reader may find it amusing to play the game backwards by reducing the present paper to seven pages of formal statements and proofs". This is still waiting to be done, though some parts of the job are written [DG, Oll-c, Oll-a, Sil03]. Full understanding and exploitation of these "contexts" will doubtlessly be an important source of new results and techniques.

The principle of the proof is as follows (and the technical conditions needed for it to work are stated below in Definition 50 and Theorem 51, extracted from [Oll-c]): Start with the free group $F_{2}$ and any family of 
expanders $\Gamma_{i}$. Put a random labelling on some subdivision $\Gamma_{1}^{/ j}$ of $\Gamma_{1}$ and let $G_{1}$ be the group given by the graphical presentation $\Gamma_{1}$. According to Proposition 47 and Theorem 46, if $j$ is large enough, $G_{1}$ will be a nontrivial hyperbolic group. As described in $\S$ III.1.a., there will be a natural graph map $\Gamma_{1}^{/ j} \rightarrow \operatorname{Cay}\left(G_{1}\right)$, which is actually a quasi-isometric embedding.

Then consider a random labelling of a subdivision of $\Gamma_{2}$ and let $G_{2}$ be the quotient of $G_{1}$ by the graphical presentation $\Gamma_{2}$. Applying the "relative" version of proposition 47 , as described in $\S$ III.1.e., $G_{2}$ will be a non-trivial hyperbolic group, provided the girth of $\Gamma_{2}$ is large compared to the hyperbolicity constant of $G_{1}$ (which is of the same order of magnitude as Diam $\Gamma_{1}$ ). Up to taking a subsequence of the family of expanders we can always suppose that girth $\Gamma_{i} \gg \operatorname{Diam} \Gamma_{i-1}$, which allows to define inductively a hyperbolic group $G_{i}$ obtained by quotienting $G_{i-1}$ by a random labelling of (a subdivision of) $\Gamma_{i}$. The group $G_{i}$ comes with a natural graph map from $\Gamma_{i}^{/ j}$ to its Cayley graph.

The group $G$ is then obtained as the direct limit of all $G_{i}$ 's. It is not finitely presented, but can be recursively presented by replacing randomness by pseudo-randomness. Indeed, the graphical small cancellation property used here is algorithmically checkable (even relatively to a given hyperbolic group!), so that we can write a program enumerating all labellings of $\Gamma_{1}$, testing whether they are $G r^{\prime}(1 / 6)$, stopping at the first such labelling found (which exists by the randomness argument), then outputting a presentation for $G_{1}$; enumerating all labellings of $\Gamma_{2}$ and testing whether they are in small cancellation relative to the explicit hyperbolic group $G_{1}$, outputting the first such labelling of $\Gamma_{2}$, etc. Note that this requires to have a recursive construction for the expanders $\Gamma_{i}$ too.

This provides a recursive enumeration of the presentation of the limit group $G$. Then applying Higman's embedding theorem (Theorem 12.18 in [Rot95], Theorem IV.7.3 in [LS77]) provides a finitely presented group $H$ in which $G$ embeds. Note that an embedding of a finitely presented group is always a uniform embedding (since there are only finitely many elements in balls of the image of the initial group), so that $H$ does not uniformly embed into the Hilbert space if $G$ does not.

The subdivision step amounts to label each edge of $\Gamma_{i}$ with a random word of length $j$ rather than with a single generator. This allows to reduce "density" of the graphical presentation, by increasing the relator length (measured by the girth) without changing the number of relators. It is very important to use the same $j$ for all the $\Gamma_{i}$ 's: indeed we only get a graph map from $\Gamma_{i}^{/ j}$ to the Cayley graph of $G$, which of course induces a map from the vertices of $\Gamma_{i}$ to $\operatorname{Cay}(G)$ with a $j$ times larger Lipschitz constant, so that if $j$ goes uncontrolled then so do the metric properties of the embedding. In other words, a bounded subdivision of a family of expanders is still a family of expanders but this is false for unbounded subdivisions. 
Another important point is that the "critical density" for non-triviality of random quotients of the $G_{i}$ 's could decrease to 0 when $i \rightarrow \infty$, thus resulting in groups that are more and more reluctant to adding new relations (forcing to increase $j$ ). As results from Theorem 40, this critical density is controlled by the spectral radius of the random walk on $G_{i}$. So it is important to get a uniform control on this spectral radius for all $G_{i}$ 's. Actually property $(T)$ of a group entails such a uniform control of the spectral radii of all of its quotients. So if $G_{1}$ has property $(T)$ we are done, and this results from Theorem 48. (Another way to proceed is to replace the initial group $F_{2}$ with a hyperbolic group having property $(T)$. Yet another, maybe most natural way is to use Theorem 42 which states that the spectral radius is almost unaffected by random quotients.)

The "density" of a graphical presentation is not only controlled by the size of cycles in the graph but also of course by the number of cycles. Demanding that these graphs have bounded geometry (valency, diameter/girth ratio) ensures that density remains bounded.

So, putting all the constraints altogether, we get the following conditions for the construction to work (see [Oll-c]). Note that having a family of expanders is not required for the process of building the limit group, so that it is possible to get Cayley graphs containing other interesting families of graphs.

Definition 50 - $A$ sequence $\left(\Gamma_{i}\right)_{i \in \mathbb{N}}$ of finite connected graphs is good for random quotients if there exists $v \geqslant 1$ and $C, C^{\prime} \geqslant 1$ such that for all $i$ we have:

- $\operatorname{girth} \Gamma_{i} \rightarrow \infty$;

- $\operatorname{Diam} \Gamma_{i} \leqslant C \operatorname{girth} \Gamma_{i}$;

- For any $x \in \Gamma_{i}, r \in \mathbb{N}$, the ball $B(x, r)$ of radius $r$ in $\Gamma_{i}$ satisfies $\# B(x, r) \leqslant C^{\prime} v^{r}$.

Theorem 51 - Let $\left(\Gamma_{i}\right)_{i \in \mathbb{N}}$ be a sequence of finite connected graphs which is good for random quotients.

Then for any $\varepsilon>0$ there exists a finitely generated group $G_{\infty}$, an increasing sequence $i_{k}$ of integers, an integer $j \geqslant 1$ and a constant $A>0$ such that, for any $k \in \mathbb{N}$, there exists a map of graphs $\varphi_{k}: \Gamma_{i_{k}}^{/ j} \rightarrow \operatorname{Cay}\left(G_{\infty}\right)$ from the $j$-subdivision of $\Gamma_{i_{k}}$ to the Cayley graph of $G_{\infty}$, which is quasi-isometric in the following sense:

For any $x, y \in \Gamma_{i_{k}}^{/ j}$ we have

$$
A\left(\operatorname{dist}(x, y)-\varepsilon \operatorname{Diam} \Gamma_{i_{k}}^{/ j}\right) \leqslant \operatorname{dist}\left(\varphi_{k}(x), \varphi_{k}(y)\right) \leqslant \operatorname{dist}(x, y)
$$

where the distance in $\Gamma_{i_{k}}^{/ j}$ is the usual graph distance and the distance in $\operatorname{Cay}\left(G_{\infty}\right)$ is that w.r.t. a fixed finite generating set. 
The group $G_{\infty}$ has some labelling of the union of the $\Gamma_{i_{k}}^{/ j}$ 's as a graphical presentation. This presentation is aspherical (in the sense given in [Oll-a]) and turns $G_{\infty}$ into a direct limit of hyperbolic groups of geometric dimension 2.

Finally, if the family of graphs $\Gamma_{i}$ is recursive, then this graphical presentation can be assumed to be recursive.

Note that the size of the fibers $\varphi_{k}^{-1}(x)$ is bounded by $j v^{\varepsilon \text { Diam } \Gamma_{i_{k}}}$, so that if $\# \Gamma_{i_{k}}$ grows reasonably fast (as is the case for expanders), then the small fiber condition appearing in [HLS02] is satisfied.

Theorem 49 now follows from the above and the existence of a recursive family of expanders (e.g. Theorems 7.4.3 and 7.4.12 of [Lub94], or [DSV03]):

THEOREM 52 - There exists a recursively enumerable family of graphs $\left(\Gamma_{i}\right)_{i \in \mathbb{N}}$ such that:

- $\# \Gamma_{i} \rightarrow \infty$;

- $\inf _{i} \lambda_{1}\left(\Gamma_{i}\right)>0$ (the $\Gamma_{i}$ are expanders);

- for all $i, \Gamma_{i}$ is regular of valency $v$;

- there exist $C_{1}, C_{2}, C_{3}$ such that $\log \# \Gamma_{i} \leqslant C_{1} \operatorname{Diam} \Gamma_{i} \leqslant C_{2} \operatorname{girth} \Gamma_{i} \leqslant$ $C_{3} \log \# \Gamma_{i}$ for all $i$.

Besides [Gro03], more information on Gromov's construction can be found in [Ghy03, Pan03, Oll-c]. Useful elements of proof appear in [DG, Oll-a, Del-b, Oll-c, Sil03] and of course in [Gro03, Gro01a, Gro01b, Gro01c]. The link with the Baum-Connes conjecture is proven in [HLS02].

\section{III.3. Kazhdan small cancellation groups?}

A more modest application of Gromov's random graphical presentations is that they allow a nice mixture of small cancellation properties and property $(T)$, using Proposition 47 together with Theorem 48. This contrasts with ordinary $C^{\prime}(1 / 6)$ groups, which do not have property $(T)$ (unless finite) by a result of Wise (Corollary 1.3 in [Wis04]).

This allows the construction of Kazhdan groups with somewhat unexpected properties, using the flexibility of small cancellation groups. The main tool here is a short exact sequence coined by Rips [Rip82]. Namely, for every countable group $Q$, Rips constructed an exact sequence $1 \rightarrow$ $N \rightarrow G \rightarrow Q \rightarrow 1$ where $G$ is a $C^{\prime}(1 / 6)$ group and the kernel $N$ is finitely generated. Pathologies of $Q$ often lift to $G$ in some way. Carefully adding a random graphical presentation to $G$ adorns $N$ with property $(T)$, namely [OW-a]:

Theorem 53 - For each countable group $Q$, there is a short exact sequence $1 \rightarrow N \rightarrow G \rightarrow Q \rightarrow 1$ such that $G$ has a $G r^{\prime}(1 / 6)$ presentation 
and $N$ has property $(T)$. Moreover, $G$ is finitely generated if $Q$ is, and $G$ is finitely presented (hence hyperbolic) if $Q$ is.

As noted by Cornulier [Cor-b], this easily implies a kind of universal property for hyperbolic Kazhdan groups:

CoRoll ARY 54 - Every countable group with property $(T)$ is a quotient of a $G r^{\prime}(1 / 6)$ hyperbolic group with property $(T)$.

Indeed, in the exact sequence above, if both $Q$ and $N$ have $(T)$ then $G$ has $(T)$ [HV89]. If $Q$ is finitely presented, then so is $G$ and thus $G$ is hyperbolic. If $Q$ is not finitely presented, a theorem of Shalom (Theorem 6.7 in [Sha00]) provides a finitely presented Kazhdan group of which $Q$ is a quotient.

Another consequence of Theorem 53 is the following. Paulin asked (open problem 5 at the end of [HV89]) if a Kazhdan group can have an infinite outer automorphism group (this is impossible for a hyperbolic group by a result of [Pau91]). Actually this can happen and more precisely [OW-a]:

Theorem 55 - Every countable group embeds in the outer automorphism group of some Kazhdan group.

Indeed in the exact sequence above, $Q$ acts on $N$ by conjugation and this action happens not to be inner. In particular for finitely presented $Q$, the group $N$ appears as a subgroup of some hyperbolic group.

Very different examples of Kazhdan groups with infinite outer automorphism groups were independently constructed by Cornulier [Cor-a] (as linear groups) and later by Belegradek and Szczepański [BSz] using relatively hyperbolic groups. Moreover Cornulier's example is finitely presented, thus answering positively a question in [OW-a].

Using the techniques in [BW05], it may be possible to show that actually every countable group is isomorphic to the outer automorphism group of some Kazhdan group. For finitely presented groups, this is shown in [BSz] up to finite index.

The main interest of the combined Rips sequence/random graphical presentation method is its flexibility. Using standard techniques it is straightforward to construct new groups with prescribed properties. In [OW-a] two easy examples are given. Recall a group $G$ is called Hopfian if every surjective homomorphism $G \rightarrow G$ is injective, and co-Hopfian if every injective homomorphism $G \rightarrow G$ is surjective.

Theorem 56 - There exists a Kazhdan group which is not Hopfian, arising as a finitely generated subgroup of a $G r^{\prime}(1 / 6)$ infinitely presented group. There exists a Kazhdan group which is not co-Hopfian, arising as a finitely generated subgroup of a $G r^{\prime}(1 / 6)$ hyperbolic group.

For comparison, for hyperbolic groups the situation is as follows: Sela proved [Sel99] that every torsion-free hyperbolic group is Hopfian, and this 
Applications: RAndom ingredients in SPECific CONSTRUCtions 67

was extended [Bum04] to any finitely generated subgroup of a torsion-free hyperbolic group (showing that the infiniteness of the presentation in the theorem above cannot be removed). Sela again (final theorem of [Sel97]) proved that a non-elementary torsion-free hyperbolic group is co-Hopfian if and only if it is freely indecomposable; hence, every Kazhdan hyperbolic group is co-Hopfian.

Once more, subsequent examples using different techniques are described in [Cor-a] and [BSz]. Noticeably, Cornulier's example of a Kazhdan nonHopfian group (arising from a $p$-arithmetic lattice) is finitely presented.

We have attempted to demonstrate that random groups already produced some interesting new examples of groups. The techniques involved are flexible enough and hopefully more is to come. 


\title{
IV. Open problems and perspectives
}

\author{
I feel, random groups altogether may grow up \\ as healthy as random graphs, for example. \\ M. Gromov, Spaces and questions
}

The problems presented hereafter are varied in style and difficulty. Some of them amount to a cleaning of results implicit in the literature, others are well-defined questions, whereas the worst of them are closer to babbling on an emerging notion. Some are directly extracted from the excellent exposition of Gromov in the final chapter of [Gro93], and still unsolved.

Only problems directly pertaining to random groups are presented here. It must be stressed that Gromov's paper [Gro03] contains a lot of new, challenging ideas inspired by his random group construction but belonging to neighboring fields, which unfortunately could not be discussed here.

Disclaimer. The list of problems is provided "as is", without any warranty, either express or implied, including, but not limited to, the warranty of correctness, of interest, of fitness to any particular purpose (such as an article or thesis), or of non-triviality. We wish the reader good luck.

IV.a. What happens at the critical density? The usual question after a talk on random groups...

Asking whether a random group at density $d=1 / 2$ is infinite or trivial might not be the right way of looking at things. The most promising and intriguing approach is to define a limit object for $\ell \rightarrow \infty$ and for definite $d<1 / 2$, and then let $d \rightarrow 1 / 2$. The limit object would be as follows: by Theorem 13 the Cayley graph of the random group $G$ is a tree up to distance $\ell(1-2 d)$, and moreover the hyperbolicity constant is at most $4 \ell /(1-2 d)$. So it is natural to consider the metric space $\frac{1}{\ell} \operatorname{Cay}(G)$ where $\frac{1}{\ell}$ means we rescale the distance by this factor: this yields, for any $\ell$, a $4 /(1-2 d)$ hyperbolic space which is a tree up to distance $1-2 d$. It seems likely that for fixed $d$, for $\ell \rightarrow \infty$ this metric space converges $\grave{a}$ la Gromov-Paulin to some (maybe deterministic in some sense) non-locally compact metric space locally modeled on a real tree. This object would depend on $d$ and be $4 /(1-2 d)$-hyperbolic. Letting then $d \rightarrow 1 / 2$ might bring a non-trivial object, maybe with some self-similarity or universality properties. 
Another approach consists in letting simultaneously $\ell \rightarrow \infty$ and $d \rightarrow 1 / 2$. Indeed, Theorem 13 shows that the ball of radius $\ell(1-2 d)$ in the Cayley graph is a tree. One is thus tempted to let $d \rightarrow 1 / 2$ and set $\ell=K /(1-2 d)$ so that the length of the smallest relation in the group is kept constant (but the big problem is that this $\ell$ may be too small for Theorem 11 to hold). It may happen that for large enough values of $K$, the group converges (in law) to some non-trivial group with radius of injectivity $K$, maybe infinitely presented. It may also happen that the group is trivial no matter how large $K$ is.

If one sticks to the question of what happens when we take exactly $d=$ $1 / 2$ in the definition of the density model, one should note the following. If in the density model we take not $(2 m-1)^{d \ell}$ but $P(\ell)(2 m-1)^{d \ell}$ relators with $P$ a subexponential term, for $d \neq 1 / 2$ this does not change the theorem. But for $d=1 / 2$ triviality or infiniteness may depend on the subexponential term $P$. It might depend moreover on the details of the model (such as taking relators on the sphere or in the ball). Exact determination of these parameters might not be very relevant. Anyway, as a short answer, for $d=1 / 2$ and $P(\ell)=1$, it is easy to check (using the probabilistic pigeonhole principle as in the comments after Theorem 11) that the random group has a positive probability (something like $(1 / e)^{2 m}$ ) to be trivial.

Some expect, however, that "all classical groups lie at $d=1 / 2$ " (using precise enough asymptotics for the $P(\ell)$ above?). By the way, note that property $(T)$ holds at $d>1 / 3$ and in particular at $d=1 / 2$.

IV.b. Different groups at different densities? Another question, lying at the core of the density model, is to know whether density really has an impact on the random group.

The question is not exactly to know whether two random groups are mutually isomorphic or not: indeed two successive random samplings of a group at the same length and density will likely be non-isomorphic (although a proof of this would be very interesting and difficult, compare $\S$ II.3. for the one-relator case; see also [Gro93], p. 279). Rather one would like to know if the probability measures for distinct values of $d$ become more and more different as $\ell \rightarrow \infty$. More precisely, one would like to know if, for every density $d_{0}$ and $\varepsilon>0$, there exists a property of groups $P_{d_{0}, \varepsilon}$ which occurs with probability tending to 1 at density $d=d_{0}$, and with probability tending to 0 at any density $d \notin\left(d_{0}-\varepsilon, d_{0}+\varepsilon\right)$, as $\ell \rightarrow \infty$.

Since a random group at density $d$ and length $\ell$ has Euler characteristic $1-m+(2 m-1)^{d \ell}$, for fixed $m$ the number $d \ell$ can be recovered from the algebraic structure of the group. So it would be enough to recover any other combination of $d$ and $\ell$ to get the answer. It is clear that as marked groups, with their standard generating set being known, random groups are different: indeed, for example the optimal isoperimetry constant $1-2 d$ is provided by Theorem 13. But changing the generating set is a mess. 
A very interesting but apparently difficult approach is suggested by Gromov in [Gro93] (p. 279). Let $G=F_{m} / N$ be a finitely presented group and define the density of this presentation as follows: there is an integer $\ell$ such that the normal closure of $N \cap B_{\ell}$ is $N$ (where $B_{\ell}$ is the ball of radius $\ell$ in $\left.F_{m}\right)$; for $1 \leqslant k \leqslant \ell$ let the density $d_{k}=\log \#\left(N \cap B_{k}\right) / \log \# B_{k}$ (one can use spheres instead of balls) and let $d\left(F_{m} \rightarrow G\right)=\sup _{k \leqslant \ell} d_{k}$. Now let $d(G)$ be the infimum of these densities $d\left(F_{m} \rightarrow G\right)$ over all finite generating sets of $G$. The question is whether for a random group at density $d$ this gives back $d$. Computation of densities of classical groups would also be interesting.

Gromov gives several other approaches in [Gro93], 9.B.(i).

The nicest thing would be to find group invariants depending continuously on density. The rank of the group is a discrete invariant varying with density, but is far from well understood (see $\S$ IV.d.). Pansu suggested the use of $\ell^{p}$-cohomology, where the critical $p$ might vary with $d$ (see [Gro93], 9.B.(i) on $\ell_{p} H^{1} \neq\{0\}$ ), or the conformal dimension of the boundary, but this approach has not yet been developed. It is wise to keep in mind the non-variation of the spectral gap (Theorem 26).

Note that this would not contradict Theorem 34 since, first, we do not expect independently picked random groups at the same density to be isomorphic, and second, random groups are not at all dense in $\mathcal{G}_{m}$.

IV.c. To $(T)$ or not to $(T)$. Property $(T)$ for random groups is known to hold at density $>1 / 3$ (Theorem 27 ), and not to hold at density $<1 / 5$ (Theorem 32). There necessarily exists a critical density for property $(T)$, since this property is inherited by quotients (indeed: if at a density $d_{0}$, property $(T)$ occurs with positive probability, then at densities $d>d_{0}$ we can write the group presentation as a union of a large number of presentations at density $d_{0}$, and one of them is enough to bring property $(T)$ ). Determination of this critical density is a frustrating question.

The gap between the Haagerup property at $d<1 / 6$ (Theorem 33) and failure of property $(T)$ at $d<1 / 5$ is probably just a technical weakness in $[\mathrm{OW}-\mathrm{b}]$. It would be very interesting to know whether, for random groups, property $(T)$ starts just where the Haagerup property stops, so that these two properties, though not opposite, would be "generically opposite" (a possibility some people consider would be "sad").

Another question is whether property $(T)$ holds at $d>1 / 3$ for a random quotient (this is already asked in [Gro87], 4.5.C). It is trivially the case for quotients by random words, for any initial group (since property $(T)$ is inherited by quotients) but in this case the random quotient might already be trivial at $d=1 / 3$ (see Theorem 40 ) and so this statement could be empty. But it is very reasonable to expect the same holds for random quotients by elements in balls of hyperbolic groups as in Theorem 38: applying the 
criterion of [Żuk03] to the generating set made of all elements of length $\ell / 3$ looks promising.

In the meantime, it is a good exercise to write a precise proof of the fact that random groups at density $d>1 / 3$ have property $(T$ ) (in the density model, not in the triangular model, see discussion in $\S$ I.3.g.).

IV.d. Rank and boundary. Even such a simple invariant of groups as the rank (minimal number of elements in a generating set) is not known for random groups (except of course for random quotients of $F_{2}$ ). The rank does vary with density: by Theorem 17 , at density 0 (and likely at small enough densities) it is equal to $m$, but it is easy to show that at density $d>1-\log (2 k-1) / \log (2 m-1)$, the rank is at most $k$ (this follows from evaluating the probability that some relator can be written as a product of one generator followed by $\ell-1$ generators chosen among $k$ ). This bound is very crude and probably not optimal (one may expect, for example, the rank to be 2 when $d \rightarrow 1 / 2$ ).

The rank would provide a non-continuous but nevertheless interesting invariant to prove that different densities produce different groups.

A possible approach is to generalize the method of Arzhantseva and Ol'shanskii at density 0 , which uses representation of subgroups by graphs and subsequent study of the exponential growth rate of the number of words which are readable on the graph. Combination of this with large deviation techniques for finite-state Markov chains may lead to a sharp estimate of the various exponents (densities) at play.

These techniques may be useful for other questions related to the algebraic structure. For example, Gromov asks ([Gro93], 9.B.(i)) whether a random group contains a non-free infinite subgroup of infinite index. Also, one would like to extend Theorem 44 on the number of one-relator groups to more relators (as asked by Gromov in [Gro93], p. 279), which, besides the interest of counting groups, would have implications for the problems discussed in § IV.b.

It is very likely that a random group is one-ended and in particular does not split as a free product (this is true at small densities and at $d>1 / 3$, see $\S$ I.3.d.). Another question is unicity of the generating set up to Nielsen moves (compare Theorem 19); e.g. for $m=2$ it can be shown that, for $d \geqslant 0.301 \ldots$ (an apparently transcendental value coming from large deviation theory of the 4-state Markov chain describing reduced words in two letters), the pair $\left(a_{1}^{2}, a_{2}\right)$ generates the random group, but it is not clear whether or not this pair is Nielsen-equivalent in the group to the standard generating pair $\left(a_{1}, a_{2}\right)$.

IV.e. More properties of random groups. Any question which is meaningful for torsion-free hyperbolic groups may be asked for random groups. Some may even be answered. 
Paragraph 1.9 of [Gro03] lists a few invariants "where a satisfactory answer seems possible": geometry of the boundary, $L_{p}$-cohomology, simplicial norm on cohomology, existence/non-existence of free subgroups, nonembeddability of random groups to each other, "something $C^{*}$-algebraic".

Another frequently asked question is the existence of non-trivial finite quotients of a random group and of residual finiteness. For any finite group $H$ fixed in advance, it is easy to show that a random group with large enough defining relators will not map onto $H$. Exchanging the limits would provide hyperbolic groups without finite quotients. (See also the temperature model in $\S$ IV.k. below.)

IV.f. The world of random quotients. The theory of random quotients of given groups, the basic idea of which is that typical elements in a given group are the most nicely behaved, is at its very beginning. (Following Erdôs, this also plays an increasingly important role in the-especially algorithmic - theory of finite groups, a subject we could not even skim over in this survey, see e.g. [Dix02].)

Theorem 38 and 40 only deal, for the moment, with quotients of torsionfree hyperbolic groups (which is nevertheless a generic class!). Of course there is no hope to extend these theorems to any initial group, if only because there exist infinite simple groups (but note that the "triviality" parts of these theorems extend to any group of exponential growth).

Nevertheless, the critical density $1 / 2$ as in Theorem 38 seems to be quite a general phenomenon. Within a hyperbolic group, the density $1 / 2$ principle might apply to random quotients by elements chosen in much more general subsets than the balls w.r.t. some generating set: more or less any large subset $X$ not resembling too much to a line should do, i.e. quotienting by less than $\sqrt{\# X}$ elements randomly chosen in $X$ should preserve hyperbolicity. The axioms defined in [Oll04] may help for this. This would have the advantage of decreasing the role of the generating set.

Identifying families of non-hyperbolic groups for which density $1 / 2$ is critical would be very nice too.

The theory of random quotients works well for torsion-free hyperbolic groups. In the case of "harmful" torsion more complex phenomena occur (§ II.2.b., [Oll05b]). Identifying necessary and sufficient conditions on torsion (probably having to do with growth/cogrowth of the centralizers of torsion elements) for the random quotient theorems to hold, and identifying the kind of new phenomena (such as more than two phases [Oll05b]) which can happen in the presence of harmful torsion, would be interesting.

Speaking of torsion, it is not even clear whether a random quotient of a torsion-free hyperbolic group is still torsion-free. It is true however that geometric dimension 2 (which implies torsion-freeness) is preserved-this is what we use in every iterated quotient construction, as in Proposition 43 and Theorems 49 and 51 for the group with expanders. Using higherdimensional complexes instead of the Cayley 2-complex (such as the Rips 
complex) or the techniques in [Del-a], may help get the result.

Theorem 39 states that the growth exponent is preserved when quotienting by random elements in a ball, and Theorem 42 states that the spectral radius is preserved when quotienting by random words; but it is likely that both are preserved whatever the model of random quotient. Elements to prove this appear in [Oll-e].

The methods used in [Oll04] to prove the phase transition theorems for random quotients of hyperbolic groups are partly geometric, partly combinatorial. On the other hand, those in [Gro03] and [DG] are almost purely geometric, but they do not allow to make it to the critical density and only work for "very" small cancellation [Del-b]. Even the basic density $1 / 2$ theorem (Theorem 11) has a much more combinatorial than geometric proof. Geometrizing these proofs is a good challenge.

IV.g. Dynamics on the space of marked groups. The bad behavior of the isomorphism relation on the space of marked groups [Ch00] from the measurable point of view suggests an ergodic approach (part 4 in [Ghy03], 9.B.(g) in [Gro93]). The dynamics here comes from the action of the Nielsen moves on $\mathcal{G}_{m}$ (more precisely, the Nielsen moves on $2 m$-tuples generate the isomorphism relation on $\mathcal{G}_{m}$ by a theorem of Tietze, see part 3 of [Ch00]). It seems likely, but is not known, that there is no non-trivial Borel measure on $\mathcal{G}_{m}$ invariant under this action. It would be nice, and perhaps important, to have an at least quasi-invariant measure.

There is a quite natural (family of) probability measure(s) on the space of all presentations of $m$-generated groups, coming from the temperature model (see $\S$ IV.k. below), which depends on a continuous parameter. This measure projects to a measure on $\mathcal{G}_{m}$, the properties of which (especially its behavior under Nielsen moves) must certainly be studied.

Besides, the study of continuity/measurability/average/whatever of the usual invariants of groups or presentations on $\mathcal{G}_{m}$ is interesting, as suggested in [Gro93], 9.B.(g).

Ghys noted that the complexity of $\mathcal{G}_{m}$ comes from lack of rigidity of the free group, and suggests that studying the space of quotients of a given marked hyperbolic group would keep all the nice properties of quotients of the free group (small cancellation, random quotients...), while maybe providing enough rigidity to allow better topological and measurable behavior, if the hyperbolic group has few automorphisms. This is of course related to $\S$ IV.f. above.

IV.h. Isoperimetry and two would-be classes of groups. Two natural properties related to isoperimetric inequalities of van Kampen diagrams arise naturally in random groups (including Gromov's group with expanders) and should be studied for themselves, independently of any probabilistic context. 
The first one is stronger than mere hyperbolicity and generalizes small cancellation. Random groups, just as small cancellation groups, have the property that any (reduced) van Kampen diagram satisfies a linear isoperimetric inequality - whereas the definition of hyperbolicity asks that only one van Kampen diagram per boundary word satisfies such an inequality. This property implies, in particular, geometric dimension 2. But it is not stable by quasi-isometry, since for example taking the Cartesian product with a finite group introduces (a finite number of) spherical diagrams.

An interesting question is whether this property can be "geometrized", i.e. to modify this property so that it becomes invariant under quasi-isometry; a way to do this may be to ask that all van Kampen diagrams, after some local modifications, satisfy the isoperimetric inequality. This geometrized property might be equivalent, for example, to having a boundary of dimension one.

This certainly has to do with the "unfolded hyperbolicity" of [Gro01c], which is Gromov's newly coined name for the "local hyperbolicity" of section 6.8.U in [Gro87] (which asks that any "locally minimal" diagram satisfies the isoperimetric inequality; a link is explained with non-existence of conformal maps, and with any surface in the space having negative Euler characteristic); these considerations probably deserve more attention.

Groups in this class may keep lots of interesting properties of small cancellation groups.

The second property is the "homogeneous isoperimetric inequality". The usual way to write the isoperimetric inequality for a van Kampen diagram $D$ is $|\partial D| \geqslant C|D|$ where $|D|$ is the number of faces of $D$. But a more natural way is a linear isoperimetric inequality between the boundary length of $D$ and the sum of the lengths of the boundary paths of faces of $D$ :

$$
|\partial D| \geqslant C \sum_{f \text { face of } D}|\partial f|
$$

which is more homogeneous since it compares a length to a length, not a length to a number. For a finite presentation the two formulations are clearly equivalent (with a loss in the constant equal to the maximal length of a relator in the presentation).

This inequality is especially useful when facing a group presentation with relators of very different lengths, and is relevant also for infinite presentations. It naturally appears in $C^{\prime}(\alpha)$ small cancellation theory (with the constant $C=1-6 \alpha$ ), in random groups (with $C=1-2 d$, see Theorem 13), in the few-relator model of random groups with various lengths (theta-condition of [Ols92]), in Champetier's work on cogrowth [Ch93], in computation of the hyperbolicity constant [Oll-b], in random quotients of hyperbolic groups (section 6.2 of [Oll04]), in iterated quotients (it is satisfied with $C=1-2 d$ under the assumptions of Proposition 43) and, 
noticeably, it is satisfied by the infinitely presented groups containing expanders constructed in [Gro03]. Maybe importantly, it allows a formulation of the local-global principle without loss in the constants and so seems to be the right assumption for it (Theorem 60 below, [Oll-f]).

So in lots of important contexts, even for finite presentations, this is the right way to write the isoperimetric inequality.

The main question is whether this has some intrinsic and/or interesting meaning for infinitely presented groups ("fractal hyperbolicity"? compare [Gro03], 1.7). The behavior under a change of presentation is unclear: for example the property is trivially satisfied (even for a finitely presented group) if the presentation consists of all relations holding in the group. One should probably restrict oneself to presentations with some minimality assumptions (e.g. the one which, given a set of generators, consists in beginning with an empty set of relators, and successively adding all relations which are not consequences of already taken, shorter relations), and study how the property is affected by elementary changes of the presentation.

IV.i. Metrizing Cayley graphs, generalized small cancellation and "rotation families". The generalized small cancellation theory used in [Gro03] (briefly described in § III.1.e. above) is developed in several papers [Gro03, Gro01a, Gro01b] (see also [DG, Del-b]). A single consolidated proof of the statements in section 2 of [Gro03] combined with a few examples (such as relative graphical small cancellation as stated in [Oll-c]) would be very useful. Compared to the traditional study of van Kampen diagrams, here the emphasis is put on geometric objects (such as lines for traditional small cancellation or trees for the graphical case) lying in a hyperbolic space acted upon by a group, and on conditions under which the space can be quotiented along these objects.

The approach can be purely geometric (as in [DG]) or in great part combinatorial (as in [Oll-a, Oll-c]). The geometric approach as written in [DG] does not work up to the optimal cancellation coefficient $1 / 6$ but only for "very small" cancellation. But its strength is that, contrary to relative small cancellation, it can deal with quotients of a hyperbolic group by relators of length equal to the characteristic length of the group plus some large constant, whereas relative small cancellation needs the relation length to be a large constant times the characteristic length of the ambient hyperbolic group. This is why this approach succeeds in the case of the Burnside group.

The main idea is to put a non-trivial, negatively curved metric on the faces of the Cayley complex. This might be a step just as important as the jump from combinatorics of words to study of Cayley graphs and van Kampen diagrams. It is advocated in [DG] that hyperbolic groups can be made "much more hyperbolic" this way, in some intrinsic sense, than when just using the edge metric on the Cayley graph (or the Euclidean metric 
on the Cayley 2-complex). This technique is very flexible and may find many applications. At the very least it should provide a nice framework to re-interpret some classical results of hyperbolic group theory in.

IV.j. Better Cayley graphs with expanders? The construction of a Cayley graph with expanders may be simplified. The most direct way would be to find an explicit $G r^{\prime}(1 / 6)$ labelling of the whole family of expanders; this would provide both a shorter proof and an isometric embedding of the expanders, instead of quasi-isometric. Getting an injective (on the vertices of the expanders) quasi-isometric embedding would already be nice.

Another "flaw" of the construction is the final step using the Higman embedding theorem in order to get a finitely presented group: this keeps nonuniform embeddability into the Hilbert space, but the quasi-isometricity of the embedding of the expanders into the Cayley graph is lost, as are geometric dimension 2 and property $(T)$, so the question of expanders quasi-isometrically contained in the Cayley graph of a finitely presented, maybe also Kazhdan group of dimension 2 is still open.

IV.k. The temperature model and local-global principles. Certainly one of the most important theoretical problems related to random groups.

All the random groups defined so far define a notion of asymptotically typical properties of groups rather than an intrinsic notion of random groups: (say in the density model) for each length $\ell$ we indeed define a measure $\mu_{\ell}$ on the set of group presentations, but this measure does not converge as $\ell \rightarrow \infty$. Rather, for a given group property $P$, its probability of occurrence under $\mu_{\ell}$ converges. As discussed in $\S$ I.4., the space of marked groups does not solve this problem because the notion of topological genericity in it is uninteresting (there are very different-looking connected components) and so an input from probability theory is required to know where in this space to look.

The temperature model, or every-length density model (discussed at the end of [Gro00], but already present in [Gro93], 9.B.(d)) attempts to solve these problems by directly defining a probability measure on the set of all (finite or infinite) group presentations, thus providing a well-defined notion of a random group. Note that this measure will project on the space of marked groups, and thus give access to the realm of infinitely presented groups.

As usual, fix a set of $m$ generators, and consider the set $F_{m}$ of reduced words. The principle is to construct a set of relators $R$ by deciding at random, for each $r \in F_{m}$, whether we put it in $R$ or not. Since there are much more long than short words, the probability to take $r$ should decrease with the length of $r$. A very natural choice is to set

$$
p(r)=(2 m-1)^{(d-1)|r|}
$$


where $|r|$ is the length of the word $r$, and $d \leqslant 1$ is a density parameter. Now, for each $r \in F_{m}$, with probability $p(r)$ we decide to put $r$ as a relator in $R$ (independently of what is decided for other $r$ 's). The random group is given by the presentation $G=\left\langle a_{1}, \ldots, a_{m} \mid R\right\rangle$.

Note that a priori $G$ is infinitely presented.

Let us interpret the parameter $d$. The expected number of words in $R$ with a given length $\ell$ is $2 m(2 m-1)^{\ell-1}(2 m-1)^{(d-1) \ell}$ because there are $2 m(2 m-1)^{\ell-1}$ reduced words of length $\ell$. Note that this behaves like $(2 m-1)^{d \ell}$ (up to a benign constant $(2 m) /(2 m-1)$ ), which is of course very reminiscent of the density model.

In other words, if for each $\ell \in \mathbb{N}^{*}, R_{\ell}^{\prime}$ is a random set of relators at density $d$ and at length $\ell$, then the union $R^{\prime}=\bigcup_{\ell \in \mathbb{N}^{*}} R_{\ell}^{\prime}$ has essentially the same probability law as $R$ (up to replacement of an average number by a fixed number of relators, which for number such as $(2 m-1)^{\ell}$ is negligible by the law of large numbers).

This justifies the name "all-length density model". The "temperature" [Gro00] refers to the idea that a word $w \in F_{m}$ has "energy" $|w|$, and so if temperature is $T$ the probability for a "random word" to be in "state" $r$ (compared to its probability to be in state $r=e$ ) is $e^{-|r| / T}$, so that $T=1 /((1-d) \log (2 m-1))$. The higher the temperature, the larger the set of relators $R$, the smaller the group $G$. When $T \rightarrow 0$, on the contrary, the set $R$ "freezes" to the empty set so that $G=F_{m}$. Note that negative densities are meaningful in this model.

As an immediate consequence of the interpretation of $d$ as a density, we get that if $d>1 / 2$ (i.e. $T \geqslant 2 / \log (2 m-1)$ ) the group $G$ is trivial with probability 1 .

In this model, for each $d>-\infty$ there is a small but definite positive probability to pick all the generators $a_{1}, \ldots, a_{m}$ and put them as relators in $R$, in which case the group is trivial. So here we do not expect a phase transition between infinity and triviality of $G$ with probabilities 0 and 1 , but rather, a phase transition between a positive probability to be infinite and a zero probability to be infinite.

Up to this remark and the fact that the presentation is infinite at $d>0$, the conjecture [Gro00] is the exact analogue of Theorem 11:

ConjeCture - If $d<1 / 2$, the group $G$ has a positive probability to be infinite, and more precisely to be a direct limit of infinite hyperbolic, torsion-free groups of geometric dimension 2.

Another way to express $d<1 / 2$ is that the function $p(r)$ is in $\ell^{2}\left(F_{m}\right)$.

Not everything can happen with positive probability: for example at $d>0$ we put an exponential number of generators, so that by a simple argument, with probability 1 the abelianization of $G$ is trivial, and so Abelian groups never appear in this model (the support of the measure is not the whole space $\left.\mathcal{G}_{m}\right)$. 
At $d<0$, by the Borel-Cantelli lemma, the presentation for $G$ is finite with probability 1 , and the model is more or less related to the few-relator model with various lengths, so that for negative densities the conjecture follows from Theorem 5 , and the group is even hyperbolic. But at $d \geqslant 0$ the presentation is infinite, and if the conjecture indeed holds the group will admit no finite presentation.

As a sidepoint, Theorem 27 implies property $(T)$ for $d>1 / 3$, with probability 1 . This property is likely to happen much earlier.

An easy but important feature [Gro00] of this model is that for any $d \geqslant 0$, with probability 1 the group $G$ has no finite quotient (compare the discussion above in $\S$ IV.e.). Indeed, let $\pi: G \rightarrow H$ be a finite quotient of $G$. The cosets $\pi^{-1}(h \in H)$ meet one element of $F_{m}$ out of $\# H$, and so for $d \geqslant 0$ it is easy to see that $R$ will contain one (actually infinitely many) element of each coset with probability 1 , thus proving that $H=\{e\}$. This was for one single finite group $H$, but the union of countably many events of probability zero has probability zero again.

The main difficulty when dealing with the temperature model is failure of the local-global principle (see one possible statement in $\S$ V., Theorem 60 , and other references a few sentences below), a.k.a. the Gromov-CartanHadamard theorem, which allows to show hyperbolicity of a group by testing only isoperimetry for van Kampen diagrams of bounded size. This implies in particular that there exists an algorithm which, given a finite group presentation, answers positively when the group is hyperbolic (but may not stop if the group is not).

When the lengths in a group presentation are of very different orders of magnitude, this principle fails (or at least no suitable version of it is known). For a fixed density $d$, for any $\ell \in \mathbb{N}$ let $R_{\ell, d}$ be a random set of relators at density $d$ and at length $\ell$. Using the axioms in [Oll04] one can show that, for any constant $A \geqslant 1$, the group presented by

$$
\left\langle a_{1}, \ldots, a_{m} \mid \bigcup_{\ell_{0} \leqslant \ell \leqslant A \ell_{0}} R_{\ell, d}\right\rangle
$$

is very probably hyperbolic, for large enough $\ell_{0}$ depending on $A$. Then, using the theory of random quotients and iterating like in Proposition 43, for any $A \geqslant 1$ we can show that if $\ell_{i+1} \gg A \ell_{i}$, the group presented by

$$
\left\langle a_{1}, \ldots, a_{m} \mid \bigcup_{i \in \mathbb{N}} \bigcup_{\ell_{i} \leqslant \ell \leqslant A \ell_{i}} R_{\ell, d}\right\rangle
$$

will very probably be infinite and a direct limit of hyperbolic groups. But the techniques used to treat $\bigcup_{\ell_{0} \leqslant \ell \leqslant A \ell_{0}} R_{\ell, d}$ are very different from those used to treat the passage from $\ell_{i}$ to $\ell_{i+1}$, so that this "lacunarity" is currently needed (see [Gro03]). Note however that this lacunarity does not (at 
least explicitly) appear in Ol'shanskiri's treatment [Ols92] of the few-relator model with various lengths (thanks to the use of a homogeneous way to write isoperimetry as discussed in $\S$ IV.h. above).

So probably the key to the temperature model is a much better understanding of the local-global principle when the relators have very different lengths. The formulation given below (Theorem 60 in $\S \mathrm{V}$.) allows some looseness for the ratio of the lengths, and a careful exploitation of it results in replacing $\ell_{0} \leqslant \ell \leqslant A \ell_{0}$ with $\ell_{0} \leqslant \ell \leqslant \ell_{0}^{\alpha}$ in the above, for some exponent $\alpha>1$. A first step would be to remove the dependency in $\ell_{2} / \ell_{1}$ in Theorem 60 [Oll-f]. But this is not enough to tackle the temperature model. The geometrizing of Cayley complexes as discussed in § IV.i. (after [Gro03], [DG] etc.) will also certainly be a key ingredient.

We refer the reader to [Gro87] (2.3.F and 6.8.M) and to [Bow91, Ols91b, Bow95, Pap96, DG, Oll-f] for more information on this important topic. There is not even a single statement unifying the various versions of the local-global principle written so far...

The same game can be played replacing $F_{m}$ with any (especially hyperbolic!) initial group $G_{0}$ and killing random elements of $G_{0}$ according to the temperature scheme, thus transposing in this model all the random quotient questions of $\S$ IV.f., and endowing some neighborhood of each group in $\mathcal{G}_{m}$ with a canonical probability measure depending on density.

IV.l. Random Lie algebras. Ask Étienne Ghys about this (see also [Gro93], 9.B.(h)).

IV.m. Random Abelian groups, computer science and statistical physics. Phase transitions arose first in statistical physics and it is natural to ask whether the phase transition of random groups does model some physical phenomenon. The answer is presently unknown.

A fundamental problem of computer science is the 3-SAT problem, which asks whether a given set of clauses on Boolean variables can be satisfied. Each clause is of the form $(\neg) x_{i} \mathrm{OR}(\neg) x_{j} \mathrm{OR}(\neg) x_{k}$, where $(\neg)$ denotes optional negations and where $1 \leqslant i, j, k \leqslant n$. A set of clauses is satisfiable if each variable can be assigned the value true or false such that all clauses become true Boolean formulae. Variants exist in which the length of the clauses is not necessarily equal to 3 . This problem is very important, and in particular it is NP-complete.

A widely used approach consists in observing the behavior of this problem for random choices of the clauses, for which methods from statistical physics are very useful (see e.g. [BCM02, MMZ01] for an introduction). In this context there is a phase transition depending on the ratio of the number of clauses to the number of Boolean variables: when this ratio is below a precise threshold the set of clauses is very probably satisfiable, whereas 
it is not above the threshold. Moreover, away from the threshold, naive algorithms perform very well though the problem is NP-complete.

This immediately brings to mind the triangular model of random groups (§ I.3.g.), which consists in taking relations of the form $x_{i}^{ \pm 1} x_{j}^{ \pm 1} x_{k}^{ \pm 1}=e$ at random and asking whether the group presented by the elements $x_{1}, \ldots, x_{n}$ subject to these relations is trivial or not. This triangular model looks strikingly like a kind of "non-commutative" version of 3-SAT.

A commonly studied toy version of the 3-SAT problem is the XOR-SAT problem, using exclusive OR's instead of OR's in the clauses. This one has a polynomial-time solution (it reduces to a linear system modulo 2), hence is considerably simpler theoretically, but nevertheless seems to keep lots of interesting properties of 3-SAT. It can be interpreted as a random quotient of the commutative group $(\mathbb{Z} / 2 \mathbb{Z})^{n}$ (or sparse random matrices), thus in line with the intuition that the triangular model is a somewhat non-commutative random 3-SAT problem.

Random 3-SAT also exhibits phases: of course the satisfiability vs. nonsatisfiability phases parallel the hyperbolicity vs. triviality phases for group, but moreover, the satisfiability phase breaks into two quite differentlybehaved subphases, one in which the set of admissible truth value assignments to the variables is strongly connected and satisfiability is easy, and one in which the set of admissible truth value assignments breaks into many well-separated clusters. These two subphases evoke the freeness vs. $(T)$ transition in the triangular model (Proposition 30 and Theorem 31): below this frontier, the group is infinite for trivial reasons, whereas above it, it is still infinite but not trivially so (compare performance of the group algorithms discussed in I.3.h.). This suggests that isolation of clusters of SAT solutions parallels isolation of the trivial representation among unitary representations of the group (one possible definition of property $(T)$ ).

The many possible assignments of truth values to the variables suggest to look not only at the random group given by a random presentation, but at all groups generated by the same elements and satisfying the random relations in the presentation (which are exactly the quotients of this group). Maybe the connectedness vs. many-clustering of solutions of SAT translates into some geometric property of the set of those groups, considered in the space $\mathcal{G}_{m}$ of marked groups (§ I.4., § IV.g.).

This is quite speculative and there may also be no relation at all between these fields. Nevertheless, methods from statistical physics and randomoriented computer science are certainly interesting tools to study for random group theorists. 


\section{Proof of the density one half theorem}

V.a. Prolegomena. Recall from the Primer that, given a group presentation, a van Kampen diagram is basically a connected planar graph each oriented edge of which bears a generator of the presentation or its inverse (with opposite edges bearing inverse generators), such that the word labelling the boundary path of each face is (a cyclic permutation of) a relator in the presentation or its inverse. The diagram is said to be reduced if moreover some kind of trivial construction is avoided. We refer to [LS77, Ols91a, Rot95] for precise definitions. The set of reduced words that are read on the external boundary path of some van Kampen diagram coincides with the set of reduced words representing the trivial element in the group.

It is known [Gro87, Sho91a] that a group $G$ is hyperbolic if and only if there exists a constant $C$ such that any reduced word $w$ representing the trivial element of $G$ appears on the boundary of some van Kampen diagram with at most $C|w|$ faces.

In particular, to establish hyperbolicity it is enough to prove that there exists a constant $\alpha>0$ such that for any diagram $D$, we have $|\partial D| \geqslant \alpha|D|$ (where $|D|$ is the number of faces of $D$ and $|\partial D|$ the length of the boundary path of $D^{1}$ ). This implies the above with $C=1 / \alpha$. Note that since reducing a van Kampen diagram preserves the boundary word, it is enough to check $|\partial D| \geqslant \alpha|D|$ for reduced diagrams (this would actually never hold for all non-reduced diagrams).

We are going to show that for a random group at density $d$ and at length $\ell$, with overwhelming probability any reduced van Kampen diagram satisfies $|\partial D| \geqslant(1-2 d-\varepsilon) \ell|D|$ (i.e. we actually prove Theorem 13$)$.

The idea is very nicely explained in [Gro93], 9.B. Remember the discussion of Gromov's density (§ I.2.): The probability that two random reduced words share a common initial subword ${ }^{2}$ of length $L$ is $1 /(2 m-1)^{L}$. So at

\footnotetext{
${ }^{1}$ which is not exactly the number of edges of $\partial D$ in case the interior of $D$ is not connected.

${ }^{2}$ Here and throughout the following we neglect the fact that for the first letter of a reduced word, we have $2 m$ choices instead of $2 m-1$ as for all subsequent letters; when dealing with cyclically reduced words, we also neglect the fact that for the last letter there may be $2 m-1$ or $2 m-2$ choices.
} 
density $d$, the probability that, in a set $R$ made of $(2 m-1)^{d \ell}$ random relators, there exist two words sharing a common initial subword of length $L$, is at most $(2 m-1)^{2 d \ell}(2 m-1)^{-L}$ (this was Proposition 10$)$.

The geometric way to think about it is to visualize a 2-face van Kampen diagram in which two faces of boundary length $\ell$ share $L$ common edges. We have shown that the probability that two relators in $R$ make such a diagram is at most $(2 m-1)^{2 d \ell-L}$ (up to an unimportant, subexponential factor $4 \ell^{2}$ accounting for the positioning and orientation of the relators in the diagram).

Now consider a random presentation $\left\langle a_{1}, \ldots, a_{m} \mid R\right\rangle$ where $R$ is made of $(2 m-1)^{d \ell}$ random reduced words of length $\ell$. Let $D$ be any van Kampen diagram made by the relators in $R$. Each internal edge of $D$ (i.e. an edge adjacent to two faces) forces an equality between two letters of the two relators read on the two adjacent faces; for random reduced words this equality has a probability $1 /(2 m-1)$ to be fulfilled. So if $L$ is the total number of internal edges in $D$, the probability that $|D|$ random reduced words fulfill the $L$ constraints imposed by $D$ is at most $1 /(2 m-1)^{L}$ (if the constraints are independent). So the probability that we can find $|D|$ relators in $R$ fulfilling the constraints of $D$ is at most $(2 m-1)^{|D| d \ell}(2 m-$ $1)^{-L}$.

Choose any $\varepsilon>0$. If $L>(d+\varepsilon)|D| \ell$, then the probability that $D$ appears as a van Kampen diagram of the presentation $R$ is less than (2m$1)^{-\varepsilon|D| \ell}$ by the reasoning above, and so when $\ell \rightarrow \infty$, with overwhelming probability $D$ does not appear as a van Kampen diagram of the random group. So we can assume $L \leqslant(d+\varepsilon)|D| \ell$.

Now we have

$$
|\partial D| \geqslant|D| \ell-2 L
$$

since $D$ has $|D|$ faces, each of length $\ell$, and each gluing between two faces decreases the boundary length by 2 . (Equality occurs when the interior of $D$ is connected; otherwise, "filaments" linking clusters of faces still increase boundary length.) Consequently, using $L \leqslant(d+\varepsilon)|D| \ell$ we get

$$
|\partial D| \geqslant|D| \ell(1-2 d-2 \varepsilon)
$$

as needed.

There are several obscure points in this proof. First, we did not justify why the constraints imposed by a van Kampen diagrams on letters of the presentation can be supposed to be independent (in fact, they are not as soon as the diagram involves several times the same relator ${ }^{3}$ ), so we are a

\footnotetext{
${ }^{3}$ The proof given in [Żuk03] for the triangular model is partly incorrect too, but in a more subtle way when a diagram involves several copies of a relator glued to itself. Namely, on page 659 of [Żuk03]: "First put in the diagram $n_{1}$ relators $r_{1}$. If they have some edges in common, denote by $l_{1}$ the length of the longest common sequence, i.e. $0 \leqslant l_{1} \leqslant 3$ " and then it is stated that, given the constraints of the diagram, the number
} 
priori not allowed to multiply all probabilities involved as we did. Second, we should exclude simultaneously all diagrams violating the isoperimetric inequality, and we only estimated the probability that one particular diagram is excluded. Third, note that the trivial group, as well as any finite group, is hyperbolic and thus satisfies the isoperimetric inequality, so we have proven that the group is hyperbolic but not necessarily infinite.

The latter is treated by a cohomological dimension argument, see below. The second problem is solved using the local-global principle of hyperbolic geometry (or Gromov-Cartan-Hadamard theorem) which will be explained later. The first point requires a more in-depth study of the probability for random relators to fulfill a diagram, which we now turn to.

V.b. Probability to fulfill a diagram. First, we need a precise definition of what it means for random words to fulfill a van Kampen diagram. We define an abstract diagram to be a van Kampen diagram in which we forget the actual relators associated with the faces, but only remember the geometry of the diagram, which faces bear the same relator as each other, the orientation of the relator of each face, and where the relators begin. Namely:

Definition $\mathbf{5 7}-A$ abstract diagram $\mathcal{D}$ is a connected planar graph without valency-1 vertices, equipped with the following data:

- An integer $1 \leqslant n \leqslant|\mathcal{D}|$ called the number of distinct relators in $\mathcal{D}$ (where $|\mathcal{D}|$ is the number of faces of $\mathcal{D}$ );

- A surjective map from the faces of $\mathcal{D}$ to the set $\{1,2, \ldots, n\}$; a face with image $i$ is said to bear relator $i$;

- For each face $f$, a distinguished edge on the boundary of $f$ and an orientation of the plane \pm 1 ; if $p$ is the boundary path of $f$ with the distinguished edge as first edge and oriented according to the orientation of $f$, we call the $k$-th edge of $p$ the $k$-th edge of $f$.

An $n$-tuple $\left(w_{1}, \ldots, w_{n}\right)$ of cyclically ${ }^{4}$ reduced words is said to fulfill $\mathcal{D}$ if the following holds: for each two faces $f_{1}$ and $f_{2}$ bearing relators $i_{1}$ and

of choices for such a relator is at most $(2 m-1)^{3-l_{1}}$.

Either $l_{1}$ denotes the maximal length of the intersection of two faces bearing $r_{1}$. In this case it is not true that the total number $L$ of internal edges of the diagram is at $\operatorname{most} \sum n_{i} l_{i}$.

Or $l_{1}$ denotes the maximal length of the intersection of a face with the union of all other faces bearing $r_{1}$. Then let $D$ be a 3-face diagram bearing three copies of $r_{1}$, the second copy having reverse orientation, and with the second letter of the first copy glued to the first letter of the second copy, and the second letter of the second copy glued to the first letter of the third copy. In this case $l_{1}=2$ but the number of choices for $r_{1}$ is $(2 m-1)^{2}$.

${ }^{4}$ Here we work with cyclically reduced words to avoid the following technical annoyance: the beginning and end of a reduced word may cancel, which forces to consider van Kampen diagrams with "inward spurs" in some faces. Anyway the theorem holds for any version, since the "probabilistic cost" of such a cancellation is identical to the probabilistic cost of a cancellation between two relators. Note however that the same 
$i_{2}$, such that the $k_{1}$-th edge of $f_{1}$ is equal to the $k_{2}$-th edge of $f_{2}$, then the $k_{1}$-th letter of $w_{i_{1}}$ and the $k_{2}$-th letter of $w_{i_{2}}$ are inverse (when the orientations of $f_{1}$ and $f_{2}$ agree) or equal (when the orientations disagree). For a $n^{\prime}$-tuple of words with $n^{\prime} \leqslant n$, define a partial fulfilling similarly.

An abstract diagram is said to be reduced if no edge is adjacent to two faces bearing the same relator with opposite orientations such that the edge is the $k$-th edge of both faces.

In other words, putting $w_{i}$ on the faces of $\mathcal{D}$ bearing relator $i$ turns $\mathcal{D}$ into a genuine van Kampen diagram.

It is clear that conversely, any van Kampen diagram defines an associated abstract diagram (which is unique up to reordering the relators). A van Kampen diagram is reduced if and only if its associated abstract diagram is.

We can choose the order of enumeration of the relators and in particular we can ask that the number of faces bearing relator $i$ is non-increasing with $i$ (call relator 1 the most frequent relator, etc.).

Note that a face of a graph can be non-trivially adjacent to itself, in which case we have $f_{1}=f_{2}$ above (but then of course $k_{1} \neq k_{2}$ ).

Hereafter we limit ourselves to abstract diagrams each face of which has boundary path of length $\ell$, in accordance with the density model of random groups. Our goal is to prove the following:

Proposition 58 - Let $R$ be a random set of relators at density $d$ and at length $\ell$. Let $D$ be a reduced abstract diagram and let $\varepsilon>0$.

Then either $|\partial D| \geqslant|D| \ell(1-2 d-2 \varepsilon)$, or the probability that there exists a tuple of relators in $R$ fulfilling $D$ is less than $(2 m-1)^{-\varepsilon \ell}$.

Note that in the "intuitive" proof above, we had a probability $(2 m-$ $1)^{-\varepsilon|D| \ell}$ instead.

To prove this proposition we shall need some more definitions. Let $n$ be the number of distinct relators in $D$. For $1 \leqslant i \leqslant n$ let $m_{i}$ be the number of times relator $i$ appears in $D$. As mentioned above, up to reordering the relators we can suppose that $m_{1} \geqslant m_{2} \geqslant \ldots \geqslant m_{n}$.

For $1 \leqslant i_{1}, i_{2} \leqslant n$ and $1 \leqslant k_{1}, k_{2} \leqslant \ell$ say that $\left(i_{1}, k_{1}\right)>\left(i_{2}, k_{2}\right)$ if $i_{1}>i_{2}$, or if $i_{1}=i_{2}$ and $k_{1}>k_{2}$. Let $e$ be an edge of $D$ adjacent to faces $f_{1}$ and $f_{2}$ bearing relators $i_{1}$ and $i_{2}$, which is the $k_{1}$-th edge of $f_{1}$ and the $k_{2}$-th edge of $f_{2}$. Say edge $e$ belongs to $f_{1}$ if $\left(i_{1}, k_{1}\right)>\left(i_{2}, k_{2}\right)$, and belongs to $f_{2}$ if $\left(i_{2}, k_{2}\right)>\left(i_{1}, k_{1}\right)$, so that an edge belongs to the second face it meets.

Note that since $D$ is reduced, each internal edge belongs to some face: indeed if $\left(i_{1}, k_{1}\right)=\left(i_{2}, k_{2}\right)$ then either the two faces have opposite orientations and then $D$ is not reduced (by definition), or they have the same

theorem does not hold for plain (non-reduced) random words, since then the combinatorics of possible cancellations is exponential, and the critical density is lower than $1 / 2$, as explaind in $\S$ II.2.a. and [Oll04]. 
orientation and the diagram is never fulfillable since a letter would have to be its own inverse.

Let $\delta(f)$ be the number of edges belonging to face $f$. For $1 \leqslant i \leqslant n$ let

$$
\delta_{i}=\max _{f \text { face bearing } i} \delta(f)
$$

which will intuitively measure the "log-probabilistic cost" of relator $i$ (lemma below).

Since each internal edge belongs to some face, we have

$$
|\partial D| \geqslant \ell|D|-2 \sum_{f \text { face of } D} \delta(f) \geqslant \ell|D|-2 \sum_{1 \leqslant i \leqslant n} m_{i} \delta_{i}
$$

LEMмA 59 - For $1 \leqslant i \leqslant n$ let $p_{i}$ be the probability that $i$ randomly chosen cyclically reduced words $w_{1}, \ldots, w_{i}$ partially fulfill $D$ (and $p_{0}=1$ ). Then

$$
p_{i} / p_{i-1} \leqslant(2 m-1)^{-\delta_{i}}
$$

The lemma is proven as follows: Suppose that $i-1$ words $w_{1}, \ldots, w_{i-1}$ partially fulfilling $D$ are given. Then, successively choose the letters of the word $w_{i}$ in a way to fulfill the diagram. Let $f$ be a face of $D$ bearing relator $i$ and realizing the maximum $\delta_{i}$.

Let $k \leqslant \ell$ and suppose the first $k-1$ letters of $w_{i}$ are chosen. If the $k$-th edge of $f$ belongs to $f$, then this means that the other face $f^{\prime}$ meeting this edge either bears a relator $i^{\prime}<i$, or bears $i$ too but the edge appears as the $k^{\prime}<k$-th edge in $f^{\prime}$ (it may even happen that $f^{\prime}=f$ ). In both cases, in order to fulfill the diagram the $k$-th letter of $w_{i}$ is imposed by the letter already present on the edge, so that choosing it at random has a probability $1 /(2 m-1)$ to be correct ${ }^{5}$. The lemma is proven.

Now for $1 \leqslant i \leqslant n$ let $P_{i}$ be the probability that there exists a $i$-tuple of words partially fulfilling $D$ in the random set of relators $R$. We trivially ${ }^{6}$ have

$$
P_{i} \leqslant(\# R)^{i} p_{i}=(2 m-1)^{i d \ell} p_{i}
$$

and according to the density philosophy, $i d \ell+\log _{2 m-1} p_{i}$ is to be seen as the dimension of the $i$-tuples of relators partially fulfilling $D$ (i.e. the $\log$ of the expected number of such $i$-tuples). This explains the role played by logs in the few next lines-beware these logs are negative!

\footnotetext{
${ }^{5}$ See footnote 4 .

${ }^{6}$ Here it is even true that $P_{i} / P_{i-1} \leqslant(\# R) p_{i} / p_{i-1}$, because $p_{i} / p_{i-1}$ is independent of the value of the words $w_{1}, \ldots, w_{i-1}$. But this is no longer true in more general contexts such as random quotients of hyperbolic groups, where one has to condition by some properties of $w_{1}, \ldots, w_{i-1}$ (the "apparent lengths" in [Oll04]).
} 
Combining Equations (2) and (3) we get

$$
\begin{aligned}
|\partial D| & \geqslant \ell|D|+2 \sum m_{i}\left(\log _{2 m-1} p_{i}-\log _{2 m-1} p_{i-1}\right) \\
& =\ell|D|+2 \sum\left(m_{i}-m_{i+1}\right) \log _{2 m-1} p_{i}
\end{aligned}
$$

and Equation (4) yields (here we use $m_{i} \geqslant m_{i+1}$ )

$$
|\partial D| \geqslant \ell|D|+2 \sum\left(m_{i}-m_{i+1}\right)\left(\log _{2 m-1} P_{i}-i d \ell\right)
$$

and observe here that $\sum\left(m_{i}-m_{i+1}\right) i d \ell=d \ell \sum m_{i}=d \ell|D|$, hence

$$
|\partial D| \geqslant \ell|D|(1-2 d)+2 \sum\left(m_{i}-m_{i+1}\right) \log _{2 m-1} P_{i}
$$

so that setting $P=\min _{i} P_{i}$ (and using $m_{i} \geqslant m_{i+1}$ again) we get

$$
\begin{aligned}
|\partial D| & \geqslant \ell|D|(1-2 d)+2\left(\log _{2 m-1} P\right) \sum\left(m_{i}-m_{i+1}\right) \\
& =\ell|D|(1-2 d)+2 m_{1} \log _{2 m-1} P \\
& \geqslant|D|\left(\ell(1-2 d)+2 \log _{2 m-1} P\right)
\end{aligned}
$$

since $m_{1} \leqslant|D|$.

Of course a diagram is fulfillable if and only if it is partially fulfillable for any $i \leqslant n$ and so

$$
\operatorname{Pr}(D \text { is fulfillable by relators of } R) \leqslant P \leqslant(2 m-1)^{\frac{1}{2}(|\partial D| /|D|-\ell(1-2 d))}
$$

which was to be proven.

V.c. The local-global principle, or Gromov-Cartan-Hadamard theorem. The proof above applies only to one van Kampen diagram. But a deep result of Gromov ([Gro87], 2.3.F, 6.8.M) states that hyperbolicity of a space can be tested on balls of finite radius. This somehow generalizes the classical Cartan-Hadamard theorem stating that a simply connected complete Riemannian manifold with non-positive sectional curvature is homeomorphic to $\mathbb{R}^{n}$.

This implies in particular that hyperbolicity is semi-testable in the sense that there exists an algorithm which, given a presentation of a hyperbolic group, outputs an upper bound for the hyperbolicity constant (but which may not stop for non-hyperbolic presentations). Such an algorithm has indeed been implemented [EH01, Hol95].

Following Gromov, the principle has been given various, effective or noneffective formulations [Bow91, Ols91b, Bow95, Pap96, DG, Oll-f]. The variant best suited to our context is the following [Oll-f]:

Theorem 60 - Let $G=\left\langle a_{1}, \ldots, a_{m} \mid R\right\rangle$ be a finite group presentation and let $\ell_{1}, \ell_{2}$ be the minimal and maximal lengths of a relator in $R$. 
For a van Kampen diagram $D$ with respect to the presentation set

$$
\mathcal{A}(D)=\sum_{f \text { face of } D}|\partial f|
$$

where $|\partial f|$ is the length of the boundary path of face $f$.

Let $C>0$. Choose $\varepsilon>0$. Suppose that for some $K$ greater than $10^{50}\left(\ell_{2} / \ell_{1}\right)^{3} \varepsilon^{-2} C^{-3}$, any reduced ${ }^{7}$ van Kampen diagram $D$ with $\mathcal{A}(D) \leqslant$ $K \ell_{2}$ satisfies

$$
|\partial D| \geqslant C \mathcal{A}(D)
$$

Then any reduced van Kampen diagram $D$ satisfies

$$
|\partial D| \geqslant(C-\varepsilon) \mathcal{A}(D)
$$

and in particular the group is hyperbolic.

Back to random groups. Here all relators in the presentation have the same length $\ell$, so that $\mathcal{A}(D)=\ell|D|$. In particular, the assumption $\mathcal{A}(D) \leqslant$ $K \ell_{2}$ in the theorem becomes $|D| \leqslant K$, i.e. we have to check diagrams with at most $K$ faces.

Choose any $\varepsilon>0$. Set $K=10^{50} \varepsilon^{-2}(1-2 d-2 \varepsilon)^{-3}$, which most importantly does not depend on $\ell$. Let $N(K, \ell)$ be the number of abstract diagrams with $K$ faces all of which have their boundary path of length $\ell$. It can easily be checked (using the Euler formula) that for fixed $K, N(K, \ell)$ grows polynomially with $\ell$ (a rough estimate yields $N(K, \ell) \leqslant \ell^{4 K} N(K)$ ).

We know (Proposition 58) that for any reduced abstract diagram $D$ fixed in advance and violating the inequality $|\partial D| \geqslant(1-2 d-2 \varepsilon) \ell|D|$, the probability that it appears as a van Kampen diagram of the presentation is at most $(2 m-1)^{-\varepsilon \ell}$. So the probability that there exists a reduced van Kampen diagram with at most $K$ faces, violating the inequality $|\partial D| \geqslant$ $(1-2 d-2 \varepsilon) \ell|D|$, is less than $N(K, \ell)(2 m-1)^{-\varepsilon \ell}$. But, for fixed $K$ and $\varepsilon$, this tends to 0 when $\ell \rightarrow \infty$ since $N(K, \ell)$ grows subexponentially with $\ell$.

So with overwhelming probability, all reduced diagrams of the presentation with at most $K$ faces satisfy the isoperimetric inequality $|\partial D| \geqslant$ $(1-2 d-2 \varepsilon) \ell|D|$. Applying Theorem 60 (with our choice of $K$ ) yields that all reduced van Kampen diagrams $D$ satisfy $|\partial D| \geqslant(1-2 d-3 \varepsilon) \ell|D|$ as needed.

The size of the constant $K$ and the large value of $N(K, \ell)$ may explain why computer experiments ( $\S$ I.3.h.) found the group to be trivial too often...

V.d. Infiniteness. The isoperimetric inequality above is shown to hold for any reduced van Kampen diagram (and not only for one van Kampen diagram per boundary word, which is enough to be hyperbolic). This

\footnotetext{
${ }^{7}$ This constraint can be weakened.
} 
implies in particular that there is no spherical diagram (a spherical diagram being a limit case of planar diagram of zero boundary length, thus violating the isoperimetric inequality) and so the Cayley 2-complex is aspherical ${ }^{8}$, hence the group has geometric (hence cohomological) dimension at most 2 . Any group with torsion has infinite cohomological dimension, and so the random group is torsion-free (which rules out non-trivial finite groups).

The trivial group is excluded since, using asphericity of the Cayley complex, the Euler characteristic of the group is equal to $1-m+\# R=$ $1-m+(2 m-1)^{d \ell}$; for positive $d$ this is $>1$, whereas the trivial group has Euler characteristic 1 (and excluding the trivial group for $d>0$ excludes it a fortiori for $d=0$ ). The elementary hyperbolic group $\mathbb{Z}$ is excluded for the same reason.

\footnotetext{
${ }^{8}$ For this to work one needs a careful definition of van Kampen diagrams, since there are several non-equivalent notions of asphericity. See e.g. the discussion in [Oll-a].
} 


\section{Bibliography}


[And58] R. D. Anderson, A characterization of the universal curve and a proof of its homogeneity, Ann. Math. 67 (1958), $\mathrm{n}^{\circ} 2,313-$ 324 .

[Ar97] G. N. Arzhantseva, On groups in which subgroups with a fixed number of generators are free (Russian), Fundam. Prikl. Mat. 3 (1997), $\mathrm{n}^{\circ} 3,675-683$.

[Ar98] G. N. Arzhantseva, Generic properties of finitely presented groups and Howson's theorem, Comm. Alg. 26 (1998), $\mathrm{n}^{\circ} 4$, 3783-3792.

[Ar00] G. N. Arzhantseva, A property of subgroups of infinite index in a free group, Proc. Amer. Math. Soc. 128 (2000), $\mathrm{n}^{\circ}$ 11, $3205-3210$.

[AC04] G. N. Arzhantseva, P.-A. Cherix, On the Cayley graph of a generic finitely presented group, Bull. Belg. Math. Soc. 11 (2004), $\mathrm{n}^{\circ} 4,589-601$.

[AL02] G. N. Arzhantseva, I. G. Lysenok, Growth tightness for word hyperbolic groups, Math. Z. 214 (2002), n 3 , 597-611.

[A096] G. N. Arzhantseva, A. Yu. Ol'shanski1, The class of groups all of whose subgroups with lesser number of generators are free is generic, Mat. Zametki 59 (1996), $\mathrm{n}^{\circ}$ 4, 489-496; English translation in Math. Notes 59 (1996), $\mathrm{n}^{\circ} 3-4,350-355$.

[BCM02] G. Biroli, S. Cocco, R. Monasson, Phase transitions and complexity in computer science: an overview of the statistical physics approach to the random satisfiability problem, Physica A 306 (2002), 381-394.

[BH99] M. R. Bridson, A. Haefliger, Metric spaces of non-positive curvature, Grundlehren der mathematischen Wissenschaften 319, Springer (1999).

[BHV] B. Bekka, P. de la Harpe, A. Valette, Kazhdan groups, book in preparation.

[BM91] M. Bestvina, G. Mess, The boundary of negatively curved groups, J. Amer. Math. Soc. 4 (1991), $\mathrm{n}^{\circ} 3,469-481$.

[Bow91] B. H. Bowditch, Notes on Gromov's hyperbolicity criterion for path-metric spaces, in Group theory from a geometrical viewpoint, ed. É. Ghys, A. Haefliger, A. Verjovsky, World Scientific (1991), 64-167. 
[Bow95] B. H. Bowditch, A short proof that a subquadratic isoperimetric inequality implies a linear one, Michigan Math. J. 42 (1995), $\mathrm{n}^{\circ} 1,103-107$.

[BŚ97] W. Ballmann, J. Świątkowski, On $L^{2}$-cohomology and property $(T)$ for automorphism groups of polyhedral cell complexes, GAFA, Geom. Funct. Anal. 7 (1997), 615-645.

[BSz] I. Belegradek, A. Szczepański, Endomorphisms of relatively hyperbolic groups, preprint, ArXiv math.GR/0501321

[Bum04] I. Bumagina, The Hopf property for subgroups of hyperbolic groups, Geom. Dedicata 106 (2004), 211-230.

[BW05] I. Bumagin, D. T. Wise, Every group is an outer automorphism group of a finitely generated group, J. Pure Appl. Algebra 200 (2005), $\mathrm{n}^{\circ} 1-2,137-147$.

[CCJJV01] P.-A. Cherix, M. Cowling, P. Jolissaint, P. Julg, A. Valette, Groups with the Haagerup property. Gromov's a-T-menability, Progress in Math. 197, Birkhäuser (2001).

[CDP90] M. Coornaert, T. Delzant, A. Papadopoulos, Les groupes hyperboliques de Gromov, Lecture Notes in Mathematics 1441, Springer (1990).

[CG05] Ch. Champetier, V. Guirardel, Limit groups as limits of free groups, Israel J. Math. 146 (2005), 1-75.

[Ch91] Ch. Champetier, Propriétés génériques des groupes de présentation finie, Ph.D. Thesis, Université Lyon 1 (1991).

[Ch93] Ch. Champetier, Cocroissance des groupes à petite simplification, Bull. London Math. Soc. 25 (1993), $\mathrm{n}^{\circ}$ 5, 438-444.

[Ch94] Ch. Champetier, Petite simplification dans les groupes hyperboliques, Ann. Fac. Sci. Toulouse Math., ser. 6, vol. 3 (1994), $\mathrm{n}^{\circ} 2,161-221$.

[Ch95] Ch. Champetier, Propriétés statistiques des groupes de présentation finie, J. Adv. Math. 116 (1995), $\mathrm{n}^{\circ} 2,197-262$.

[Ch00] Ch. Champetier, L'espace des groupes de type fini, Topology 39 (2000), $\mathrm{n}^{\circ} 4,657-680$.

[CM88] A. Connes, H. Moscovici, Conjecture de Novikov et groupes hyperboliques, C. R. Acad. Sci. Paris Sér. I Math. 307 (1988), $\mathrm{n}^{\circ} 9,475-480$. 
[CM90] A. Connes, H. Moscovici, Cyclic cohomology, the Novikov conjecture and hyperbolic groups, Topology 29 (1990), $\mathrm{n}^{\circ} 3,345-$ 388.

[CMV04] P.-A. Cherix, F. Martin, A. Valette, Spaces with measured walls, the Haagerup property and property $(T)$, Ergodic Theory Dynam. Systems 24 (2004), ${ }^{\circ} 6,1895-1908$.

[CN] I. L. Chatterji, G. A. Niblo, From wall spaces to $C A T(0)$ cube complexes, to appear in Internat. J. Algebra Comput. special issue, ArXiv math.GT/0309036

[Coh82] J. M. Cohen, Cogrowth and amenability of discrete groups, J. Funct. Anal. 48 (1982), 301-309.

[Cor-a] Y. de Cornulier, Finitely presentable, non-Hopfian groups with Kazhdan's Property $(T)$ and infinite outer automorphism group, to appear in Proc. Amer. Math. Soc., ArXiv math.GR/0502140

[Cor-b] Y. de Cornulier, A note on quotients of word hyperbolic groups with Property $(T)$, preprint, ArXiv math.GR/0504193

[CS98] P.-A. Cherix, G. Schaeffer, An asymptotic Freiheitssatz for finitely generated groups, Enseign. Math. (2) 44 (1998), $\mathrm{n}^{\circ} 1-2$, $9-22$.

[CV96] P.-A. Cherix, A. Valette, On spectra of simple random walks on one-relator groups, Pacific J. Math. 175 (1996), $\mathrm{n}^{\circ}$ 2, 417-438.

[Del96a] T. Delzant, Sous-groupes distingués et quotients des groupes hyperboliques, Duke Math. J. 83 (1996), $\mathrm{n}^{\circ} 3,661-682$.

[Del96b] T. Delzant, Décomposition d'un groupe en produit libre ou somme amalgamée, J. Reine Angew. Math. 470 (1996), 153180.

[Del-a] T. Delzant, Asphéricité et sous-groupes libres des groupes hyperboliques, manuscript (1996).

[Del-b] T. Delzant, Mesoscopic curvature and very small cancellation theory, manuscript (2002).

[DG] T. Delzant, M. Gromov, Groupes de Burnside et géométrie hyperbolique, preprint.

[Dix02] J. D. Dixon, Probabilistic group theory, C. R. Math. Acad. Sci. Soc. R. Can. 24 (2002), $\mathrm{n}^{\circ} 1,1-15$. 
[DSV03] G. Davidoff, P. Sarnak, A. Valette, Elementary number theory, group theory, and Ramanujan graphs, London Mathematical Society Student Texts 55, Cambridge University Press, Cambridge (2003).

[EH01] D. B. A. Epstein, D. Holt, Computation in word-hyperbolic groups, Internat. J. Algebra Comput. 11 (2001), n 4, 467-487.

[EH] D. B. A. Epstein, D. Holt, The linearity of the conjugacy problem in word hyperbolic groups, to appear in Internat. J. Algebra Comput.

[GhH90] É. Ghys, P. de la Harpe, Sur les groupes hyperboliques d'après Mikhael Gromov, Progress in Math. 83, Birkhäuser (1990).

[Ghy90] É. Ghys, Les groupes hyperboliques, séminaire Bourbaki 722 (1990). Published in Astérisque 189-190 (1990), 203-238.

[Ghy03] É. Ghys, Groupes aléatoires, séminaire Bourbaki 916 (2003). Published in Astérisque 294 (2004), 173-204.

[GH97] R. I. Grigorchuk, P. de la Harpe, On problems related to growth, entropy, and spectrum in group theory, Dynam. Control Systems 3 (1997), $\mathrm{n}^{\circ} 1,51-89$.

[Gri80] R. I. Grigorchuk, Symmetrical random walks on discrete groups, in Multicomponent random systems, ed. R. L. Dobrushin, Ya.G. Sinai, Adv. Prob. Related Topics 6, Dekker (1980), 285-325.

[Gri84] R. I. Grigorchuk, Degrees of growth of finitely generated groups and the theory of invariant means, Izv. Akad. Nauk SSSR Ser. Mat. 48 (1984), $\mathrm{n}^{\circ}$ 5, 939-985; English translation in Math. USSR-Izvestiya 25 (1985), $\mathrm{n}^{\circ} 2,259-300$.

[Gro78] M. Gromov, Hyperbolic manifolds, groups and actions, in Riemann surfaces and related topics. Proceedings of the 1978 Stony Brook Conference (State Univ. New York, Stony Brook, N.Y., 1978), Ann. of Math. Stud. 97, Princeton University Press, Princeton (1981), 183-213.

[Gro81] M. Gromov, Groups of polynomial growth and expanding maps, Inst. Hautes Études Sci. Publ. Math. 53 (1981), 53-73.

[Gro83] M. Gromov, Infinite groups as geometric objects, in Proceedings of the International Congress of Mathematicians, Vol. 1, 2, Warsaw (1983), 385-392, PWN, Warsaw, 1984.

[Gro87] M. Gromov, Hyperbolic groups, in Essays in group theory, ed. S. M. Gersten, Springer (1987), 75-265. 
[Gro93] M. Gromov, Asymptotic invariants of infinite groups, in Geometric group theory, ed. G. Niblo, M. Roller, Cambridge University Press, Cambridge (1993).

[Gro00] M. Gromov, Spaces and questions, GAFA 2000 (Tel Aviv, 1999), Geom. Funct. Anal. Special Volume (2000), Part I, 118161.

[Gro01a] M. Gromov, Mesoscopic curvature and hyperbolicity, in Global differential geometry: the mathematical legacy of Alfred Gray, ed. M. Fernández, J. A. Wolf, Contemporary Mathematics 288, American Mathematical Society (2001), 58-69.

[Gro01b] M. Gromov, CAT $(\kappa)$-spaces: construction and concentration, Zap. Nauchn. Sem. S.-Peterburg. Otdel. Mat. Inst. Steklov (POMI) 280 (2001), Geom. i Topol. 7, 100-140, 299-300; English translation in J. Math. Sci. (N. Y.) 119 (2004), $\mathrm{n}^{\circ}$ 2, 178-200.

[Gro01c] M. Gromov, Small cancellation, unfolded hyperbolicity, and transversal measures, in Essays on geometry and related topics, Mémoires dédiés à André Haefliger, ed. É. Ghys, P. de la Harpe, V. F. R. Jones, V. Sergiescu, T. Tsuboi, Monograph. Enseign. Math. 38, L'Enseignement mathématique, Genève (2001), vol. 2, 371-399.

[Gro03] M. Gromov, Random walk in random groups, GAFA, Geom. Funct. Anal. 13 (2003), $\mathrm{n}^{\circ} 1,73-146$.

[Gup89] N. Gupta, On groups in which every element has finite order, Amer. Math. Monthly 96 (1989), $\mathrm{n}^{\circ} 4,297-308$.

[Guy] L. Guyot, Estimations de dimensions de Minkowski dans l'espace des groupes marqués, preprint.

[Har00] P. de la Harpe, Topics in geometric group theory, Chicago University Press (2000).

[Hig98] N. Higson, The Baum-Connes conjecture, in Proceedings of the International Congress of Mathematicians, Vol. II (Berlin, 1998), Doc. Math. 1998, Extra Vol. II, 637-646 (electronic).

[HLS02] N. Higson, V. Lafforgue, G. Skandalis, Counterexamples to the Baum-Connes conjecture, GAFA, Geom. Funct. Anal. 12 (2002), $\mathrm{n}^{\circ} 2,330-354$.

[Hol95] D. Holt, KBMAG-Knuth-Bendix in Monoids and Automatic Groups, software package (1995), available at http: //homepages . maths . warwick . ac.uk/ dfh/ 
[Hol00] D. Holt, Word-hyperbolic groups have real-time word problem, Internat. J. Algebra Comput. 10 (2000), $\mathrm{n}^{\circ}$ 2, 221-227.

[HP98] F. Haglund, F. Paulin, Simplicité de groupes d'automorphismes d'espaces à courbure négative, Geom. Topol. Monograph 1 (1998), 181-248.

[HV89] P. de la Harpe, A. Valette, La propriété $(T)$ de Kazhdan pour les groupes localement compacts, Astérisque 175, Soc. Math. Fr. (1989).

[HW] G. C. Hruska, D. T. Wise, Axioms for finiteness of cubulations, preprint (2004).

[Iva94] S. V. Ivanov, The free Burnside groups of sufficiently large exponents, Internat. J. Algebra Comput. 4 (1994), $\mathrm{n}^{\circ} 1-2,1-$ 308.

[Iva98] S. V. Ivanov, On the Burnside problem for groups of even exponent, in Proceedings of the International Congress of Mathematicians, Vol. II (Berlin, 1998), Doc. Math. 1998, Extra Vol. II, 67-75 (electronic).

[IO96] S. V. Ivanov, A. Yu. Ol'shanskiY, Hyperbolic groups and their quotients of bounded exponents, Trans. Amer. Math. Soc. 348 (1996), $\mathrm{n}^{\circ} 6,2091-2138$.

[Kes59] H. Kesten, Symmetric random walks on groups, Trans. Amer. Math. Soc. 92 (1959), 336-354.

[KL05] M. Kreck, W. Lück, The Novikov conjecture. Geometry and algebra, Oberwolfach Seminars 33, Birkhäuser (2005).

[KMSS03] I. Kapovich, A. Myasnikov, P. Schupp, V. Shpilrain, Genericcase complexity, decision problems in group theory, and random walks, J. Algebra 264 (2003), $\mathrm{n}^{\circ} 2,665-694$.

[KMSS05] I. Kapovich, A. Myasnikov, P. Schupp, V. Shpilrain, Averagecase complexity and decision problems in group theory, Adv. Math. 190 (2005), $\mathrm{n}^{\circ} 2,343-359$.

[KS05] I. Kapovich, P. Schupp, Genericity, the ArzhantsevaOl'shanskit method and the isomorphism problem for onerelator groups, Math. Ann. 331 (2005), $\mathrm{n}^{\circ}$ 1, 1-19.

[KS] I. Kapovich, P. Schupp, Delzant's T-invariant, Kolmogorov complexity and one-relator groups, to appear in Comm. Math. Helvetici, ArXiv math.GR/0305353 
[KSS] I. Kapovich, P. Schupp, V. Shpilrain, Generic properties of Whitehead's algorithm and isomorphism rigidity of random one-relator groups, to appear in Pacific J. Math., ArXiv math.GR/0303386

[Laf02] V. Lafforgue, $K$-théorie bivariante pour les algèbres de Banach et conjecture de Baum-Connes, Invent. Math. 149 (2002), $\mathrm{n}^{\circ} 1$, 1-95.

[LS77] R. C. Lyndon, P. E. Schupp, Combinatorial group theory, Ergebnisse der Mathematik und ihrer Grenzgebiete 89, Springer (1977).

[Lub94] A. Lubotzky, Discrete groups, expanding graphs and invariant measures, Progress in Math. 125, Birkhäuser (1994).

[MMZ01] O. C. Martin, R. Monasson, R. Zecchina, Statistical mechanics methods and phase transitions in optimization problems. Phase transitions in combinatorial problems (Trieste, 1999), Theoret. Comput. Sci. 265 (2001), n $1-2,3-67$.

[MY02] I. Mineyev, G. Yu, The Baum-Connes conjecture for hyperbolic groups, Invent. Math. 149 (2002), $\mathrm{n}^{\circ}$ 1, 97-122.

[Nic04] B. Nica, Cubulating spaces with walls, Algebr. Geom. Topol. 4 (2004), 297-309 (electronic).

[NR98] G. A. Niblo, M. A. Roller, Groups acting on cubes and Kazhdan's property $(T)$, Proc. Amer. Math. Soc. 126 (1998), $\mathrm{n}^{\circ} 3$, 693-699.

[Oll04] Y. Ollivier, Sharp phase transition theorems for hyperbolicity of random groups, GAFA, Geom. Funct. Anal. 14 (2004), $\mathrm{n}^{\circ} 3$, 595-679.

[Oll05a] Y. Ollivier, Cogrowth and spectral gap of generic groups, Ann. Inst. Fourier (Grenoble) $\mathbf{5 5}$ (2005), $\mathrm{n}^{\circ} 1,289-317$.

[Oll05b] Y. Ollivier, Collapsing of random quotients of hyperbolic groups with torsion, C.R. Math. Acad. Sci. Paris 341 (2005), 137-140.

[Oll-a] Y. Ollivier, On a small cancellation theorem of Gromov, to appear in Bull. Belg. Math. Soc., ArXiv math.GR/0310022

[Oll-b] Y. Ollivier, Growth exponent of generic groups, to appear in Comment. Math. Helv., ArXiv math.GR/0401050

[Oll-c] Y. Ollivier, Cayley graphs containing expanders, after Gromov, manuscript (2003). 
[Oll-d] Y. Ollivier, Spectral interpretations of Property $(T)$, manuscript (2003).

[Oll-e] Y. Ollivier, Remarques sur la croissance et la cocroissance des groupes aléatoires, informal notes (2004).

[Oll-f] Y. Ollivier, Some small cancellation properties of random groups, to appear in Internat. J. Algebra Comput., ArXiv math.GR/0409226

[Ols82] A. Yu. Ol'shanskiľ, The Novikov-Adyan theorem, Mat. Sb. (N.S.) $118(1982), \mathrm{n}^{\circ}$ 2, 203-235. English translation in Math. $\mathrm{USSR}-\mathrm{Sb}$

[Ols83] A. Yu. Ol'shanskir, On a geometric method in the combinatorial group theory, in Proceedings of the International Congress of Mathematicians, Vol. 1, 2 (Warsaw, 1983), 415-424, PWN, Warsaw, 1984.

[Ols91a] A. Yu. Ol'shanskiı̌, Geometry of defining relations in groups (translated from the 1989 Russian original), Mathematics and its Applications (Soviet Series) 70, Kluwer Academic (1991).

[Ols91b] A. Yu. Ol'shanski1, Hyperbolicity of groups with subquadratic isoperimetric inequality, Internat. J. Algebra Comput. 1 (1991), $\mathrm{n}^{\circ} 3,281-289$.

[Ols91c] A. Yu. Ol'shanski1, Periodic quotient groups of hyperbolic groups, Mat. Sb. 182 (1991), $\mathrm{n}^{\circ}$ 4, 543-567; English translation in Math. USSR-Sb. $72(1992), \mathrm{n}^{\circ} 2,519-541$.

[Ols92] A. Yu. Ol'shanskiı̌, Almost every group is hyperbolic, Internat. J. Algebra Comput. 2 (1992), $\mathrm{n}^{\circ} 1,1-17$.

[Ols93] A. Yu. Ol'shanskiǔ, On residualing homomorphisms and Gsubgroups of hyperbolic groups, Internat. J. Algebra Comput. 3 (1993), $\mathrm{n}^{\circ} 4,365-409$.

[OW-a] Y. Ollivier, D. T. Wise, Kazhdan groups with infinite outer automorphism group, to appear in Trans. Amer. Math. Soc., ArXiv math.GR/0409203

[OW-b] Y. Ollivier, D. T. Wise, Cubulating groups at density $1 / 6$, preprint.

[Pan98] P. Pansu, Formules de Matsushima, de Garland et propriété $(T)$ pour des groupes agissant sur des espaces symétriques ou des immeubles, Bull. Soc. Math. Fr. 126 (1998), ${ }^{\circ}$ 1, 107-139. 
[Pan03] P. Pansu, Groupes aléatoires, lecture at the Soc. Math. Fr. annual meeting (2003), available at http://www.math.u-psud. $\mathrm{fr} / \sim$ pansu/liste-prepub.html

[Pap96] P. Papasoglu, An algorithm detecting hyperbolicity, in G. Baumslag (ed.) et al., Geometric and computational perspectives on infinite groups, DIMACS Ser. Discrete Math. Theor. Comput. Sci. 25 (1996), 193-200.

[Pau91] F. Paulin, Outer automorphisms of hyperbolic groups and small actions on R-trees, in Arboreal group theory (Berkeley, CA, 1988), Math. Sci. Res. Inst. Publ. 19, Springer, New York (1991), 331-343.

[Pau03] F. Paulin, Sur la théorie élémentaire des groupes libres (d'après Sela), séminaire Bourbaki 922 (2003). Published in Astérisque 294 (2004), 363-402.

[Rip82] E. Rips, Subgroups of small cancellation groups, Bull. London Math. Soc. 14 (1982), $\mathrm{n}^{\circ} 1,45-47$.

[Rot95] J. J. Rotman, An introduction to the theory of groups, fourth edition, Graduate Texts in Mathematics 148, Springer (1995).

[Sag95] M. Sageev, Ends of group pairs and non-positively curved cube complexes, Proc. London Math. Soc. (3) 71 (1995), $\mathrm{n}^{\circ} 3,585-$ 617.

[Sel92] Z. Sela, Uniform embeddings of hyperbolic groups in Hilbert spaces, Israel J. Math. 80 (1992), $\mathrm{n}^{\circ}$ 1-2, 171-181.

[Sel95] Z. Sela, The isomorphism problem for hyperbolic groups, I, Ann. of Math. (2) 141 (1995), $\mathrm{n}^{\circ} 2,217-283$.

[Sel97] Z. Sela, Structure and rigidity in (Gromov) hyperbolic groups and discrete groups in rank 1 Lie groups, II, GAFA, Geom. Funct. Anal. 7 (1997), $\mathrm{n}^{\circ} 3,561-593$.

[Sel99] Z. Sela, Endomorphisms of hyperbolic groups, I: The Hopf property, Topology 38 (1999), $\mathrm{n}^{\circ} 2,301-321$.

[Sel] Z. Sela, Diophantine geometry over groups, VI: The elementary theory of a free group, to appear in GAFA, Geom. Funct. Anal.

[Ser77] J.-P. Serre, Arbres, amalgames, $\mathrm{SL}_{2}$, Astérisque 46, Soc. Math. Fr. (1997).

[Sha00] Y. Shalom, Rigidity of commensurators and irreducible lattices, Invent. Math. 141 (2000), $\mathrm{n}^{\circ} 1,1-54$. 
[Sho91a] H. Short et al., Notes on word hyperbolic groups, in Group theory from a geometrical viewpoint, ed. É. Ghys, A. Haefliger, A. Verjovsky, World Scientific (1991), 3-63.

[Sho91b] H. Short, Quasiconvexity and a theorem of Howson's, in Group theory from a geometrical viewpoint, ed. E. Ghys, A. Haefliger, A. Verjovsky, World Scientific (1991), 168-176.

[Shu99] A. G. Shukhov, On the dependence of the growth exponent on the length of the defining relation, Math. Notes $65(1999), \mathrm{n}^{\circ} 3_{-}$ 4, 510-515.

[Sil03] L. Silberman, Addendum to: "Random walk in random groups", GAFA, Geom. Funct. Anal. 13 (2003), n 1, 147-177.

[Ska99] G. Skandalis, Progrès récents sur la conjecture de BaumConnes. Contribution de Vincent Lafforgue, séminaire Bourbaki 829 (1999). Published in Astérisque 276 (2002), 105-135.

[Sta83] J. Stallings, Topology of finite graphs, Invent. Math. 71 (1983), $\mathrm{n}^{\circ} 3,551-565$.

[Sti82] J. Stillwell, The word problem and the isomorphism problem for groups, Bull. Amer. Math. Soc. (N.S.) 6 (1982), n 1, 33-56.

[STY02] G. Skandalis, J.-L. Tu, G. Yu, The coarse Baum-Connes conjecture and groupoids, Topology 41 (2002), $\mathrm{n}^{\circ} 4,807-834$.

[Swa96] G. A. Swarup, On the cut point conjecture, Electron. Res. Announc. Amer. Math. Soc. 2 (1996), $\mathrm{n}^{\circ}$ 2, 98-100 (electronic).

[TV99] S. Thomas, B. Velickovic, On the complexity of the isomorphism relation for finitely generated groups, J. Algebra 217 (1999), $\mathrm{n}^{\circ} 1,352-373$.

[Val02a] A. Valette, Nouvelles approches de la propriété $(T)$ de Kazhdan, séminaire Bourbaki 913 (2002). Published in Astérisque 294 (2004), 97-124.

[Val02b] A. Valette, Introduction to the Baum-Connes conjecture, from notes taken by Indira Chatterji, with an appendix by Guido Mislin, Lectures in Mathematics ETH Zürich, Birkhäuser (2002).

[Ver00] A. M. Vershik, Dynamic theory of growth in groups: entropy, boundaries, examples, Russian Math. Surveys 55 (2000), $\mathrm{n}^{\circ} 4$, 667-733. 
[Wan98] M.-T. Wang, A fixed point theorem of discrete group actions on Riemannian manifolds, J. Differential Geom. 50 (1998), $\mathrm{n}^{\circ} 2$, 249-267.

[Wis04] D. T. Wise, Cubulating small cancellation groups, GAFA, Geom. Funct. Anal. 14 (2004), n 1, 150-214.

[Wis] D. T. Wise, Sixtolic complexes and their fundamental groups, preprint.

[Woe00] W. Woess, Random walks on infinite graphs and groups, Cambridge Tracts in Mathematics 138, Cambridge University Press (2000).

[Yu00] G. Yu, The coarse Baum-Connes conjecture for spaces which admit a uniform embedding into Hilbert space, Invent. Math. $139(2000), \mathrm{n}^{\circ} 1,201-240$.

[Żuk96] A. Żuk, La propriété $(T)$ de Kazhdan pour les groupes agissant sur les polyèdres, C. R. Acad. Sci. Paris Sér. I Math. 323 (1996), $\mathrm{n}^{\circ} 5,453-458$.

[Żuk03] A. Żuk, Property $(T)$ and Kazhdan constants for discrete groups, GAFA, Geom. Funct. Anal. 13 (2003), $\mathrm{n}^{\circ} 3,643-670$.

Yann Ollivier

CNRS, UMPA, École normale supérieure de Lyon

46 , allée d'Italie

69007 Lyon, FRANCE

yann.ollivier@umpa.ens-lyon.fr

http://www. umpa.ens-lyon.fr/ yollivie/ 